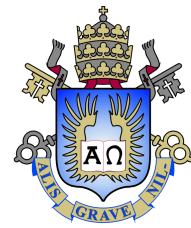

Davi Zerpini Mecler

\title{
A Metaheuristic for the Pipe Laying Support Vessels Scheduling Problem
}

Dissertation presented to the Programa de Pós-graduação em Engenharia de Produção of PUC-Rio in partial fulfillment of the requirements for the degree of Mestre em Engenharia de Produção.

Advisor : Prof. Rafael Martinelli Pinto

Co-advisor:

Prof. Arild Hoff

Rio de Janeiro

April 2020 


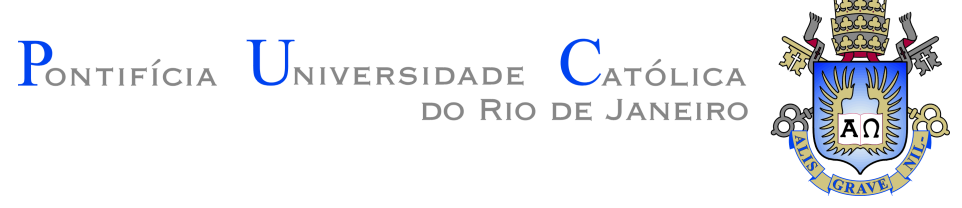

Davi Zerpini Mecler

\section{A Metaheuristic for the Pipe Laying Support Vessels Scheduling Problem}

Dissertation presented to the Programa de Pós-graduação em Engenharia de Produção of PUC-Rio in partial fulfillment of the requirements for the degree of Mestre em Engenharia de Produção. Approved by the Examination Committee.

Prof. Rafael Martinelli Pinto

Advisor

Departamento de Engenharia Industrial - PUC-Rio

Prof. Arild Hoff

Co-advisor

Molde University College - HiMolde

Prof. Silvio Hamacher

Departamento de Engenharia Industrial - PUC-Rio

Prof. Thibaut Victor Gastón Vidal

Departamento de Informática - PUC-Rio

Rio de Janeiro, April 24, 2020 
All rights reserved.

\section{Davi Zerpini Mecler}

Bachelor in Industrial Engineering (2017) at the Pontifical Catholic University of Rio de Janeiro (PUC-Rio). Earned a scholarship by $\mathrm{CNPq}$ for the masters. Worked at a state logistics company before entering the PUC-Rio masters program. The author works as a researcher at Tecgraf Institute since June 2019, supporting decision makers in the Oil \& Gas Industry.

Bibliographic data

Mecler, Davi Zerpini

A Metaheuristic for the Pipe Laying Support Vessels Scheduling Problem / Davi Zerpini Mecler; advisor: Rafael Martinelli Pinto ; co-advisor: Arild Hoff. - 2020.

75 f.: il. color. ; $30 \mathrm{~cm}$

Dissertação (Mestrado) - Pontifícia Universidade Católica do Rio de Janeiro, Departamento de Engenharia Industrial, 2020.

Inclui bibliografia

1. Engenharia Industrial - Teses. 2. Escalonamento de Máquinas Paralelas Idênticas. 3. Family Setup Times. 4. Iterated Local Search. 5. Cadeia de Suprimentos de Óleo e Gás. I. Martinelli Pinto, Rafael. II. Hoff, Arild. III. Pontifícia Universidade Católica do Rio de Janeiro. Departamento de Engenharia Industrial. IV. Título. 


\section{Acknowledgments}

Many people and institutions have contributed for this work development and I will use this space to mention and thank all of them. First I want to thank my advisor Rafael Martinelli, for all the knowledge, support and friendship he gave me through all this thesis development, spending many hours in discussions and suggestions for the improvement of this work. Silvio Hamacher who, together with Rafael Martinelli, proposed to me this thesis theme and invited me to work at Tecgraf Institute, where I learn very much everyday and flourished the knowledge described in this work. My coadvisor Arild Hoff and professor Irina Gribkovskaia, who received me in Norway, at Molde University College, where I learned a lot, and helped me to improve my skills and this thesis text.

I want to thank all my colleagues in Tecgraf, who inspire me every day, creating a motivating working environment, helping me to evolve as student and human being. Some of these colleagues have direct contribution on this study and I must cite them here. Gabriela Ribas and Pedro Lobato, who coordinate the project I am inserted, intimately related to this thesis development, Victor Abu and Janaina Marchesi, who have taught me many skills in optimization methods and programming, being crucial for this thesis evolution, Iuri Santos, Luana Carrilho and Rachel Ventriglia, who also help me to improve as a researcher. I must thank my family, Ian Mecler, Elizabeth Zerpini, Jordana Mecler, Katia Mecler, Rosinha Goldenstein, Nair Semblano e Edir Semblano for all the love and support I have received through all my life. Jordana Mecler in special helped me through this thesis, as she is an engineer colleague and taught me many programming skills.

This work was supported by PUC-Rio, Tecgraf Institute and the studied company, providing an extremely healthy environment for the development of this thesis. I would like to thank some of my professors: Hugo Repolho, who first invited me to enter the Industrial Engineer masters program, Mario Bittencourt, who taught me very much about logistics and transportation, Fernando Cyrino, Luciana Pessoa, Julia Fleck, Felipe Scavarda and José Eugenio, for all the transmitted knowledge. Also I must mention my masters and Tecgraf colleagues: Thiago Augusto, who is a great friend and student, Isabella Fischer, João Gelli, Bruno Mariani, Luiza Fiorencio, Danuza Dreux, Igor Peres, Leonardo Bastos, Bianca Brandão, Leila Dantas, Amanda Batista, Adrian Perez, Guilherme Faveret, Ricardo Terzian and Samir Azzan.

At last I want to thank my friends Miguel, Nicolas, Edgard, Arthur, Rafael, Lucca, Daniel, Beatriz, Gabriel, Pedro, Rodrigo, Luis Filipe, João Felipe, Tom and many others.

This study was financed in part by the Coordenação de Aperfeiçoamento de 
Pessoal de Nível Superior - Brasil (CAPES) - Finance Code 001 and I am very grateful for this support.

Also I would like to thank the Conselho Nacional de Desenvolvimento Científico e Tecnológico $(\mathrm{CNPq})$, who provided my master's program scholarship and PUC-Rio where I have lived great moments and learned more than I could imagine. 


\section{Abstract}

Mecler, Davi Zerpini; Martinelli Pinto, Rafael (Advisor); Hoff, Arild (Co-Advisor). A Metaheuristic for the Pipe Laying Support Vessels Scheduling Problem. Rio de Janeiro, 2020. 75p. Dissertação de Mestrado - Departamento de Engenharia Industrial, Pontifícia Universidade Católica do Rio de Janeiro.

This work objective is to propose an Iterated Local Search metaheuristic to minimize the weighted completion time of jobs on identical parallel machines scheduling problems. The secondary objective of this work is to propose a practical solution to the real problem of the studied company. The motivation of this work consists on a practical application in the Oil \& Gas industry, where the PLSV vessels perform ordered operations in a set of wells aiming to maximize the oil production. The characteristics of the problem such as: eligibility between vessels and operations, setup times related to the family of activities, association between operations and wells and setup times depending on the material arrival fit well the identical parallel machine schedule modelling. In this work, an introduction about the theme is presented, followed by a literature review on identical parallel machine scheduling problems, the mathematical formulation with the problem description is presented, the methodology, including the solution representation, constructive heuristic, neighborhood structures, local search and Iterated Local Search is exposed, at last, the method results and conclusions of the work are summarized.

\section{Keywords}

Metaheuristic Parallel Machine Scheduling Oil \& Gas PLSV Iterated Local Search 


\section{Resumo}

Mecler, Davi Zerpini; Martinelli Pinto, Rafael; Hoff, Arild. Uma Metaheuristica para o Problema de Escalonamento de Pipe Laying Support Vessels. Rio de Janeiro, 2020. 75p. Dissertação de Mestrado - Departamento de Engenharia Industrial, Pontifícia Universidade Católica do Rio de Janeiro.

Este trabalho tem como objetivo propor uma metaheurística Iterated Local Search para minimizar o tempo de término ponderado de jobs em problemas de escalonamento de máquinas idênticas paralelas. O objetivo secundário do trabalho é propor uma solução prática para um problema real da companhia estudada em questão. A motivação para o trabalho consiste em uma aplicação prática na indústria de óleo e gás, onde os navios PLSV realizam operações em poços de forma ordenada e visando a antecipação dos poços de petróleo mais produtivos. As características do problema, tais quais: elegibilidade entre navio e operações, tempos de setup relativos à família de atividades, associações entre operações e poços e setups que dependem da chegada de material se adequam a modelagem baseada em escalonamento de máquinas paralelas idênticas. No trabalho uma introdução à respeito do tema é apresentada, em seguida é feita uma revisão da literatura sobre problemas de máquinas paralelas idênticas, a formulação matemática com a descrição do problema é apresentada, a metodologia contemplando representação da solução, heuristica construtiva, vizinhanças exploradas, busca local e Iterated Local Search é exposta, por fim são apresentados os resultados do método e as conclusões sobre o trabalho.

\section{Palavras-chave}

Metaheurística Escalonamento de Máquinas Paralelas Óleo \& Gás PLSV Iterated Local Search 


\section{Table of contents}

$\begin{array}{llr}1 & \text { Introduction } & \mathbf{1 1}\end{array}$

2 Literature Review $\quad 16$

3 Mathematical Formulation $\quad 21$

3.1 Problem Description 21

3.2 Positional Scheduling Formulation 23

4 Methodology $\quad 26$

4.1 Solution Representation And Evaluation 26

4.2 Constructive Heuristic $\quad 27$

4.2.1 Constructive Heuristic Decision 32

4.3 Neighborhoods 34

4.3.1 Erase Function 34

4.3.2 Insert Function $\quad 35$

4.3.3 Relocate Neighborhood 38

$\begin{array}{lll}\text { 4.3.4 Swap Neighborhood } & 38\end{array}$

4.3.5 Setup Insertion and Setup Removal Neighborhoods 40

4.3.6 Infeasibility Strategy 41

4.4 Local Search 42

4.4.1 First Improvement 42

4.4.2 Random Variable Neighborhood Descent 43

4.5 Iterated Local Search 43

4.5.1 Perturbation $\quad 45$

4.5.2 Acceptance Criteria $\quad 45$

$5 \quad$ Computational Experiments $\quad 47$

5.1 Instances Description 47

5.2 Parameters 48

$\begin{array}{lll}5.3 & \text { Results } & 48\end{array}$

5.4 Statistical Tests $\quad 53$

6 Conclusions and Future Work $\quad 56$

A Detailed Results of the Methods for each Instance 61 


\section{List of figures}

Figure 1.1 The PLSV Connections Scheme 12

Figure 1.2 Manifold Connections 12

Figure 1.3 Water Injection Scheme 13

Figure 1.4 Production Wells and Water Injection Wells Pipelines 14

Figure 4.1 Solution Representation and Starting Times 27

Figure 4.2 Schedule Construction Procedure Scheme 29

Figure 4.3 First Allocation on Schedule Construction Procedure 30

Figure 4.4 Second Allocation on Schedule Construction Procedure 31

Figure 4.5 Third Allocation on Schedule Construction Procedure 31

Figure 4.6 Fourth Allocation on Schedule Construction Procedure 32

Figure 4.7 Fifth Allocation on Schedule Construction Procedure 32

Figure 4.8 Erase Function - Single Operation Batch 35

Figure 4.9 Erase Function - Multiple Operation Batch 35

Figure 4.10 Insert Function - case 1

Figure 4.11 Insert Function - case $2 \quad 36$

Figure 4.12 Insert Function - case $3 \quad 37$

Figure 4.13 Insert Function - case $4 \quad 37$

Figure 4.14 Insert Function - case 5 37

Figure 4.15 Relocate Neighborhood - case 1

Figure 4.16 Relocate Neighborhood - case $2 \quad 39$

Figure 4.17 Swap Neighborhood - case 1

Figure 4.18 Swap Neighborhood - case 2

Figure 4.19 Setup Insertion 41

Figure 4.20 Setup Removal 41

Figure 5.1 Boxplot - All Groups $\quad 52$

Figure 5.2 Dominance Among Methods 53 


\section{List of tables}

Table 2.1 Summary of Identical Parallel Machine Scheduling Studies 20

$\begin{array}{lll}\text { Table } 4.1 & \text { Operations Data } & 29\end{array}$

Table 4.2 Machines Data 29

Table 5.1 Results for Group $1 \quad 49$

Table 5.2 Results for Group $2 \quad 49$

Table 5.3 Results for Group $3 \quad 50$

Table 5.4 Results for Group $4 \quad 50$

Table 5.5 Results for Group 5 51

Table 5.6 Results for Group $6 \quad 51$

Table 5.7 ANOVA Tests Within All Pairs of Methods 54

Table A.1 Complete Results for each Method and Instance 61 


\section{Introduction}

Pipe Laying Support Vessels (PLSV) are one of the most critical agents on the oil offshore exploration and production $(\mathrm{E} \& \mathrm{P})$. These vessels are responsible for connecting sub-sea oil wells to the production platforms. Their high operational cost is reflected in expensive daily fleet rates Bremenkamp (2017). Efficiently planning these connections is a crucial step on the oil \& gas supply chain management, once this is the last stage before a well can start the oil production. The exploration companies have an available fleet of these vessels to coordinate and need to define a schedule for each PLSV to perform interconnection operations at a set of oil wells located off-shore. Any improvement on this schedule is reverted in a great amount of money saved, which can be measured by oil production, fleet rental cost, tardiness in the whole operation and other key factors. One or more of these objectives must be considered for the company to optimize their schedule providing a relevant competitive advantage.

The PLSV operation starts at the port, with the loading of the necessary equipment to perform the connection procedures. Once the vessel is loaded, it navigates to reach the off-shore wells where the operations are performed. The PLSV connects the lines between the platform and the wells and then returns to the port to reload and repeat this process to perform more operations. This whole cycle is planned for a one-year time horizon in most cases. An illustration of the platform, lines and wells is shown in Figure 1.1

There are three main families of operations that the PLSV perform: wells interconnections, which are the most relevant and common activities; manifold installations, allowing a single production platform to be connected with many oil wells; and scheduled vessel maintenance, which are related to the vessel and not the wells but is considered as an operation in the schedule. An image of a manifold installation is exposed in Figure 1.2.

Regarding the vessels, each one has a specific release date, due to contract characteristics. PLSV vessels can be eligible or not to perform a specific operation since their fleet is heterogeneous. Another aspect to be considered is the occupancy capacity, rated on the vessel's deck that will be used to store the necessary equipment. 


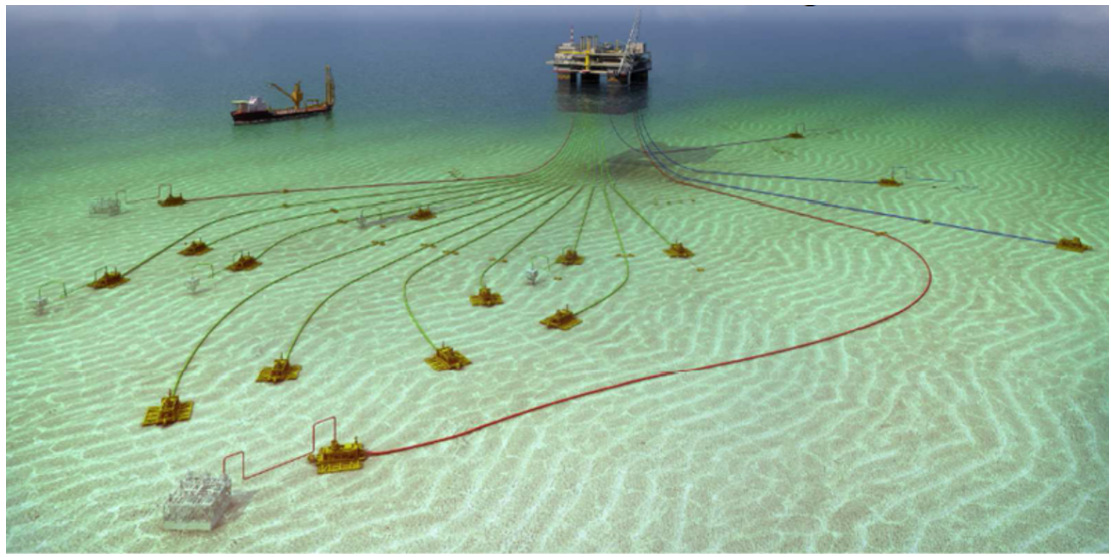

Figure 1.1: The PLSV Connections Scheme

Source: CENPES/Petrobras

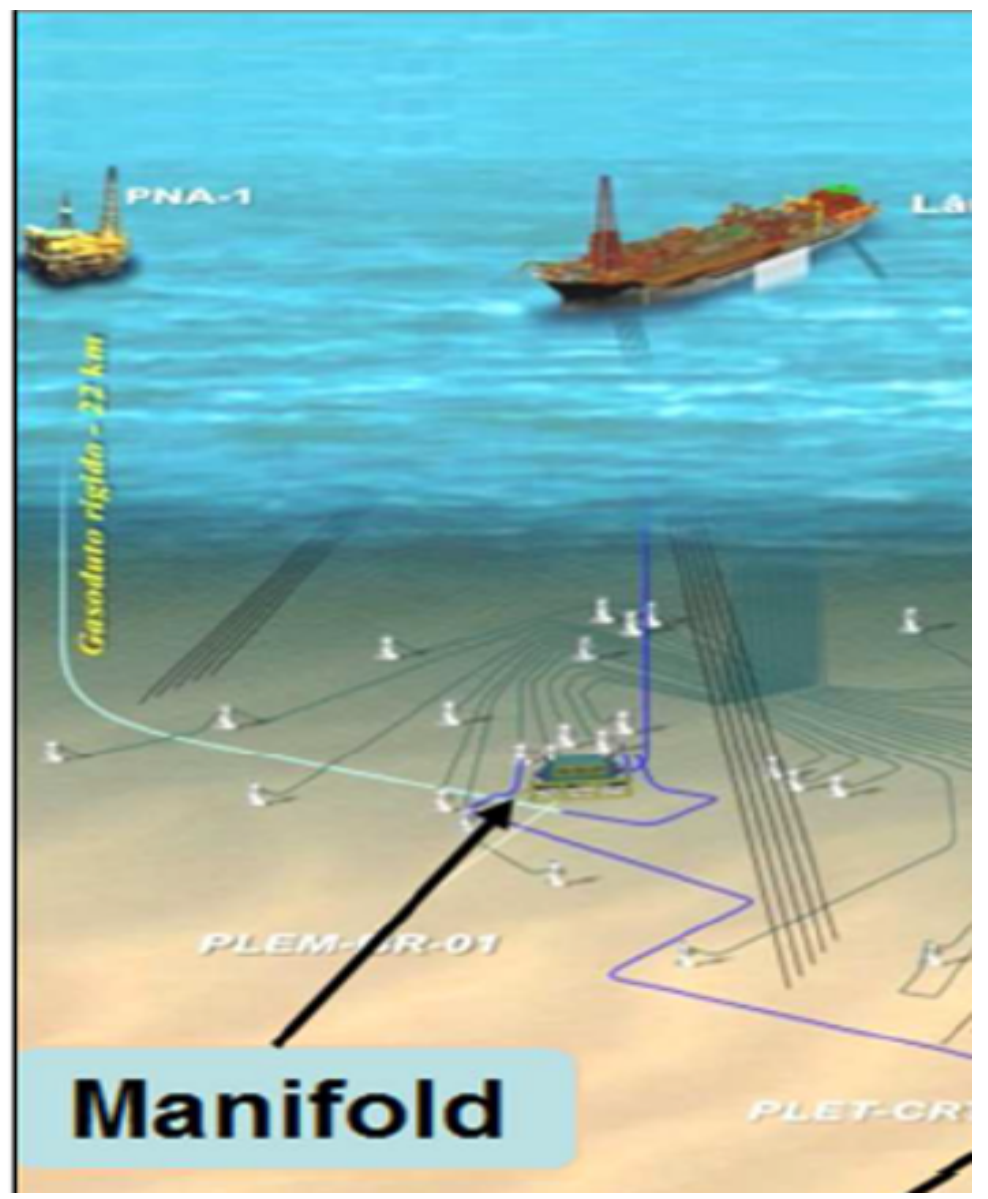

Figure 1.2: Manifold Connections

Source: CENPES/Petrobras

In Figure 1.3, a water injection well illustration is shown. The oil production wells can be rising wells, that have enough pressure to elevate the oil from the reservoir, and the non-rising wells, that need lifting methods such as water and gas injection to pressurize the well and elevate the oil. 
Regarding the petroleum production, there are two types of wells that the PLSV operate on: the oil production wells, which are responsible for the oil elevation from the reservoir to the platform and the water/gas injection wells, that energize the reservoir, guaranteeing a constant pressure after the oil removal.

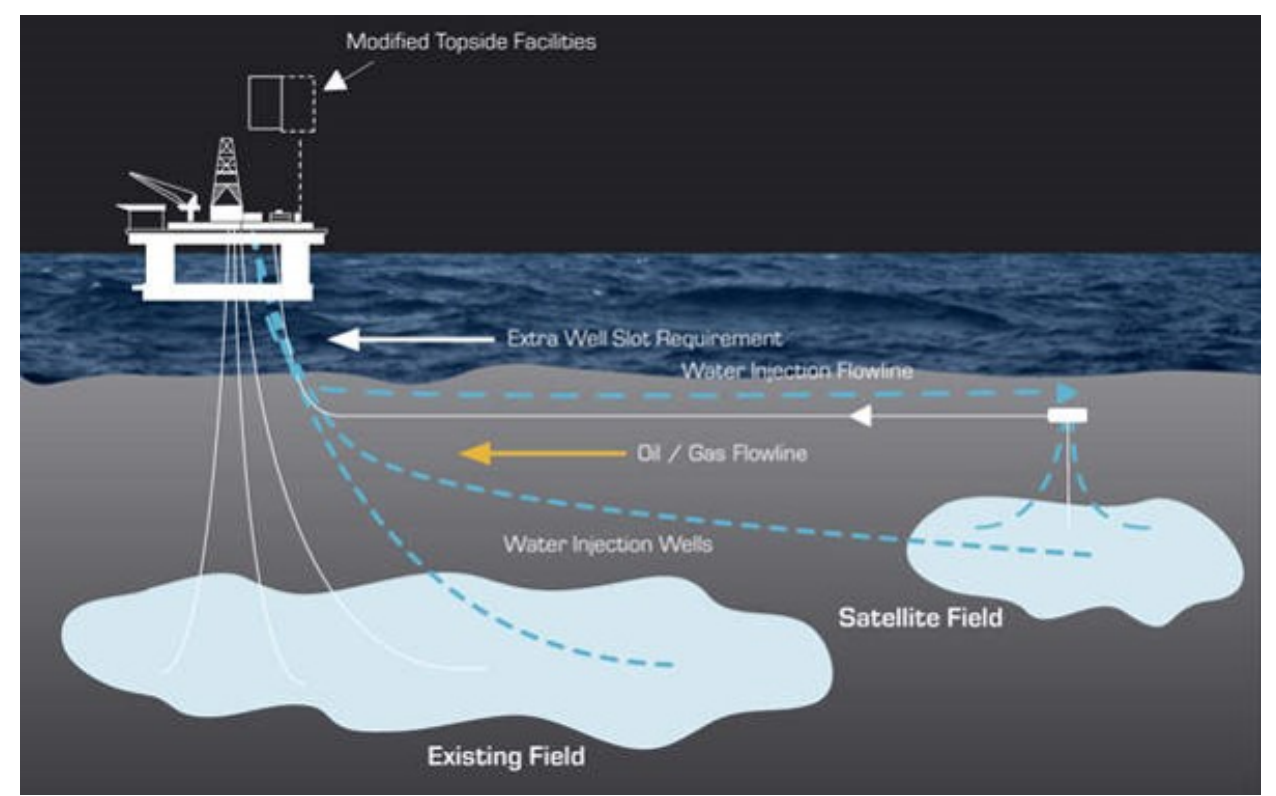

Figure 1.3: Water Injection Scheme

Source: Offshore Technology

For the wells to be considered complete, different kinds of operations need to be performed. These operations have release dates, related to the arrival of the necessary equipment, and estimated processing times. The production rates in each well varies and there is dependence between them, where a well needs others to be finished in order to start the oil production. The oil production wells have three kinds of line that the PLSV will connect to the platform: the production line, which will transport the petroleum from the reservoir to the platform, the umbilical line that opens and shuts the wells valves, and the annular lines that are used to pressurize the wells and elevate the oil. The water and gas injection wells require two kinds of pipelines: the annular line and the umbilical line. These lines are explained in Figure 1.4.

The PLSV is an extremely limited and expensive resource, influencing decision makers to search for the available tools to optimize their activities. The problem studied in this work consists on delivering a schedule plan that determines: which operations will be performed by each vessel, the date and order of these operations and how this schedule will be divided in PLSV voyages. The structure of these voyages consists on a setup time, that considers the loading time of the necessary equipment and navigation time to the wells, 

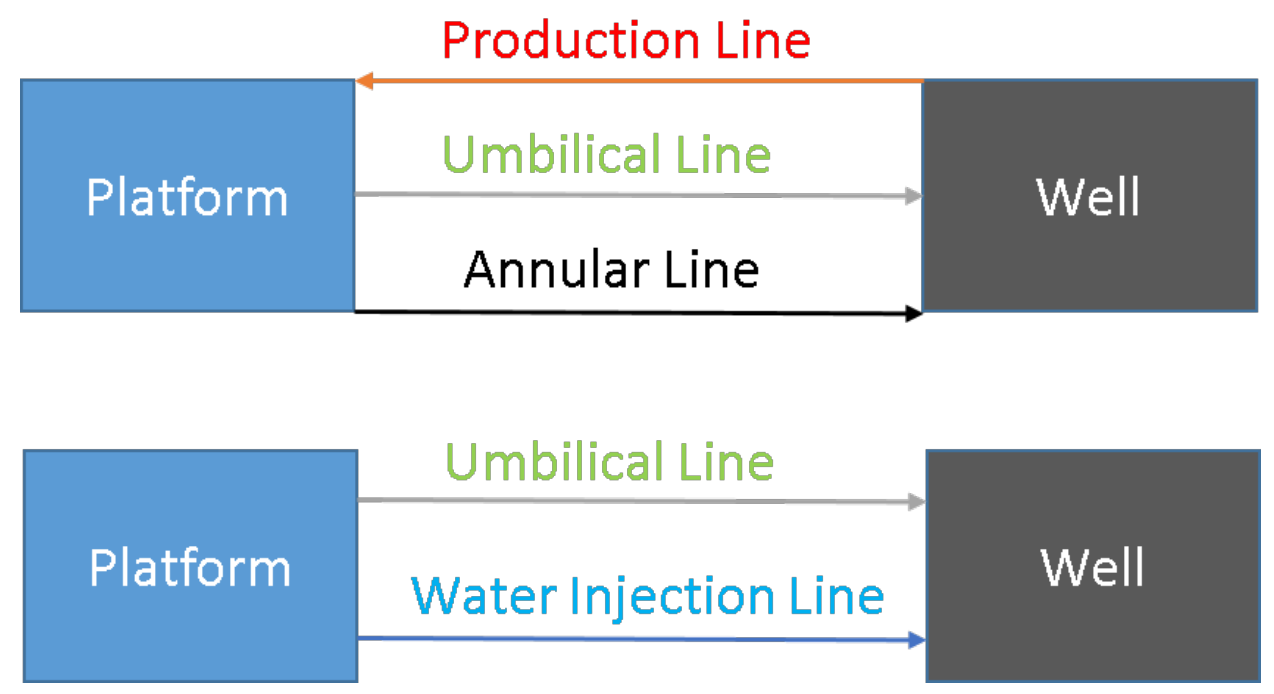

Figure 1.4: Production Wells and Water Injection Wells Pipelines

Source: The Author

followed by one or more operations of the same family to be performed. These setup times are classified as non-anticipatory because they can only start when all operations of the voyage are released (due to equipment availability). The objective is finishing the most productive wells as soon as possible, maximizing the oil production.

Due to the characteristics of the PLSV scheduling problem, we can model it as an identical parallel machine scheduling problem. The vessels are interpreted as machines that perform operations. Each operation is associated to a well, which we will call a job, following the common parallel machine scheduling problem notation. In that notation, the operations are the elements that are processed by the machines, while the jobs are associated to a set of operations. In that way, the jobs are not processed directly by machines, but when all operations associated to a specific job are finished, it is considered complete. The family of operations is related to their types and for each family we have a different setup time associated. Another important aspect of the machine scheduling problem is the association between the batches and PLSV voyages. The batches are formed by one setup time followed by a sequence of operations. In the PLSV problem any voyage starts with loading the vessels and navigating to the wells (analogous to the machine scheduling setup times) to perform a sequence of operations.

Identical parallel machine scheduling has been intensively studied in the past 20 years, in many different applications and approaches. The objective of this work is to develop efficient algorithms to minimize the weighted completion time on identical parallel machine scheduling problems with non-anticipatory 
family setup times, eligibility between machines and operations, release dates of operations and machines, job-operations associations and non-preemptive operations. The secondary objective of this work is to solve the real-life application that motivated this study which is the PLSV scheduling problem.

The main contributions of this work is providing a local search procedure as well as a metaheuristic algorithm that reach high quality solutions for the described problem in competitive time. Another important contribution is running extent computational experiments in a large set of instances based in real PLSV problem data.

The remainder of this work is divided as follows. In Chapter 2, a complete literature review on identical machine scheduling problem is presented. In Chapter 3, we describe the problem and present a mathematical formulation in detail. In Chapter 4, the methodology of the work is introduced, including the implemented constructive heuristic, explored neighborhood structures, the local search procedure and the Iterated Local Search metaheuristic. In Chapter 5, computational experiments are shown with structured results and in Chapter 6 , we present the conclusions of the thesis and recommended future work. 


\section{2}

\section{Literature Review}

The parallel machine scheduling problem with setup times has been intensively studied in the literature for the past 20 years. In this study we focus only on the identical parallel machine scheduling problem with setup times, but there are several other considerations on non-identical parallel machine problems, with and without setup times and other modelling approaches. Potts and Kovalyov (2000) provided a extensive literature review on scheduling with batching, not only in parallel machines, explaining the basic algorithms and focused on dynamic programming methods to solve this kind of problems. These algorithms consist in creating a forward recursion appending jobs in a specific schedule and a backward recursion inserting jobs in the beginning of a schedule. We suggest this article to understand problems that treat non-identical parallel machine scheduling.

We selected 21 of the most relevant papers aimed to solve identical parallel machine scheduling problems with family setup times. The solution approaches presented vary from mixed-integer linear problem formulations to heuristics and metaheuristics. Some of the articles are focused on real life problems applications while most of them are based on theoretical problems. We limited our research to articles published in the last twenty years, written in English, related to identical parallel machine scheduling problems and published in journals or book series. The search was performed on the Scopus platform and the key words searched were: "identical parallel machine scheduling problem" and "batch scheduling problem" A summary of all studied publications is shown in Table 2.1.

Regarding exact methods, Webster and Azizoglu (2001) addresses the problem on scheduling identical parallel machines with family setup times in order to minimize total weighted flowtime. They also present two dynamic programming algorithms, named backward and forwards procedures. They show that when the number of machines and families are fixed, the backward recursion is executed in polynomial time in the sum of the weights and the forward recursion is executed in polynomial time in the sum of times (both setup and processing). Comparing then the sum of weights and times will define which 
method will be more effective for each problem. Chen and Powell (2003) propose a column generation and branch-and-bound techniques to solve both sequencedependent and independent problems on identical parallel machines. These techniques consist in solving linear relaxations of the problem by standard column generation procedures. The focus of the method is based on node selection and branching variable selection to effectively solve these relaxations. They aim to solve both minimizing total weighted completion time problems and minimizing tardiness. Azizoglu and Webster (2003) also propose branchand-bound algorithms to solve identical parallel machine scheduling problems with family setup times, but the objective function is to minimize total weighted flowtime. The authors take in consideration some dominance properties based on the shortest weighted processing time (SWPT) order and lower bound definitions to propose an efficient branch-and-bound method. Dunstall and Wirth (2005b) improve the branch-and-bound methods aiming to minimize the weighted completion time by proposing a different branching scheme able to shorten the search tree size. They propose two branch-and-bound algorithms that use different branching schemes. They also use techniques to eliminate redundancies on solutions and consider upper bounds by scheduling entire families into machines with a SWPT rule. Dunstall and Wirth (2005a) propose a method to solve the problem with sequence-dependent setup times. They develop a four stage algorithm by mustering all jobs of a family in a single batch and allocating to machines, re-sequencing the batches at single machines, splitting the batches and at last performing a single machine sequencing phase. These steps are based on dominance rules between jobs and families. Omar and Teo (2006) develop a mixed-integer programming approach to minimize both earliness or tardiness on identical parallel machine scheduling with sequencedependent setup times. Basically, they improve a formulation by adding a constraint ensuring that a job and its successor will be produced in the same machine.

Regarding heuristics and metaheuristics applied to similar problems, Min and Cheng (1999) propose a genetic algorithm based on machine code to minimize the makespan in identical parallel machine scheduling problems. In the coding phase the gene code is also the number of the machine which the job is processed, reason why the method is named genetic algorithm based on machine code. After the genetic algorithm, the authors develop a simulated annealing metaheuristic. Mendes et al. (2002) compare the performance of two different metaheuristic methods to minimize makespan in identical parallel machine scheduling with family setup times. The first metaheuristic explored is a tabu search with four different types of insertion/removal moves. The second 
is a memetic approach combining a population-based method with local search procedures. Ruiz and Maroto (2005) work consists on reviewing and comparing heuristics and metaheuristics for the permutation flowshop problem to minimize makespan. In the paper, they approach 25 different methods, varying from the most antique and classic heuristics to some recent metaheuristics, including genetic algorithms, tabu searches, iterated local searches and hybrid techniques. Liao et al. (2012) studied the identical parallel machine scheduling problem with setup times focused on minimizing the weighted completion time. The authors propose an heuristic that aims to decrease the setup times and balance the weighted completion time on each machine. There are two steps involved: the first is to use the a tabu search to schedule jobs in each machine. The second contrasts the obtained schedule with another without setup times by changing the assignment of jobs to machines. These exchanges are subject to certain conditions based on dominance rules such as the SWPT Weighted Mean Processing Time. Schaller (2014) presents a set of procedures for scheduling identical parallel machines with family setup times focused on minimizing total tardiness. They test these procedures in a large set of instances and conclude that for this specific problem, the genetic algorithms are usually more effective. Mehdizadeh et al. (2015) develop a metaheuristic algorithm called vibration damping optimization (VDO), based on mechanical vibration, to solve the identical parallel machine scheduling problem with sequence-independent setup times. The VDO algorithm consists in iteratively and randomly changing the current solution based on oscillating the solution around an amplitude parameter. Van Der Zee (2015) proposes a simulation study on family-based dispatching heuristics to identical machine scheduling. They test and compare existing heuristics for the problem and create new heuristics by extending the existing ones, simulating the results on a large set of instances. Wu et al. (2018) introduced a modified Water Flow-Like Algorithm (WFA) to solve the identical parallel machine scheduling problem with sequence-dependent setup times, considering due dates, order profit and tardiness penalties. Their strategy was to develop a procedure similar to the variable neighborhood search and mix it with the WFA, by splitting and moving operations. Dipak and Gupta (2018) presented an improved cuckoo search algorithm (ICSA) focused on minimizing makespan on identical parallel machine scheduling. Their first step consists on generating a set of initial population schedules, combining the longest processing time rule with job-interchange procedures, and selecting the best schedule. The authors then propose a heuristic approach to transform continuous positions in the CSA into discrete job schedules. At last, an heuristic algorithm related to pairwise exchange neighborhood is introduced to complete the ICSA procedure. 
Nguyen et al. (2018) propose an heuristic of layering and adapting (HLA) to minimize both energy consumption and makespan in identical parallel machine scheduling problems. The algorithm strategy is to consider resource allocations of jobs as flexible form articles to be packed in a strip with variant heights throughout its length. Two local search procedures are used with the concepts of adapting process and greedy repackage process.

Considering different real-life applications of the identical parallel machine scheduling problem, Shin and Leon (2004) applies a scheduling approach to module processing in thin film transistor liquid crystal display manufacturing. The authors develop a multifit heuristic and a tabu search to solve the problem focused on minimizing total tardiness and minimizing total family setups. Ciavotta et al. (2016) propose dynamic heuristics and candidates reduction strategies with the multiheuristic rollout procedures, applying them in illustrative case studies. The case study was first proposed by Pacciarelli et al. (2011) and is based on a pharmaceutical manufacturing plant. Kaplan and Rabadi (2011) studies the aerial refuelling scheduling problem with release dates, and due date deadline windows, focused on minimizing the total weighted tardiness. A simulated annealing metaheuristic was proposed as well as a metaheuristic with randomized priority search, using an apparent priceswise tardiness cost with ready times as a dispatching rule. Lee (2017) studies a real life production scheduling from a plant of Acrylonitrile-Butadiene-Styrene plate products. The focus is to minimize tardiness and the strategy developed proposes a dispatching rule to provide an initial solution and then applying an iterated greedy search metaheuristic to improve the results. Abu-Marrul et al. (2020a)studies the PLSV scheduling problem. Three mixed-integer linear formulations are proposed to solve the problem aiming to minimize completion time: the positional, the batch, and the time-index models. Abu-Marrul et al. (2020b) also studies the PLSV scheduling problem. A constructive heuristic is presented to minimize the weighted completion time. 


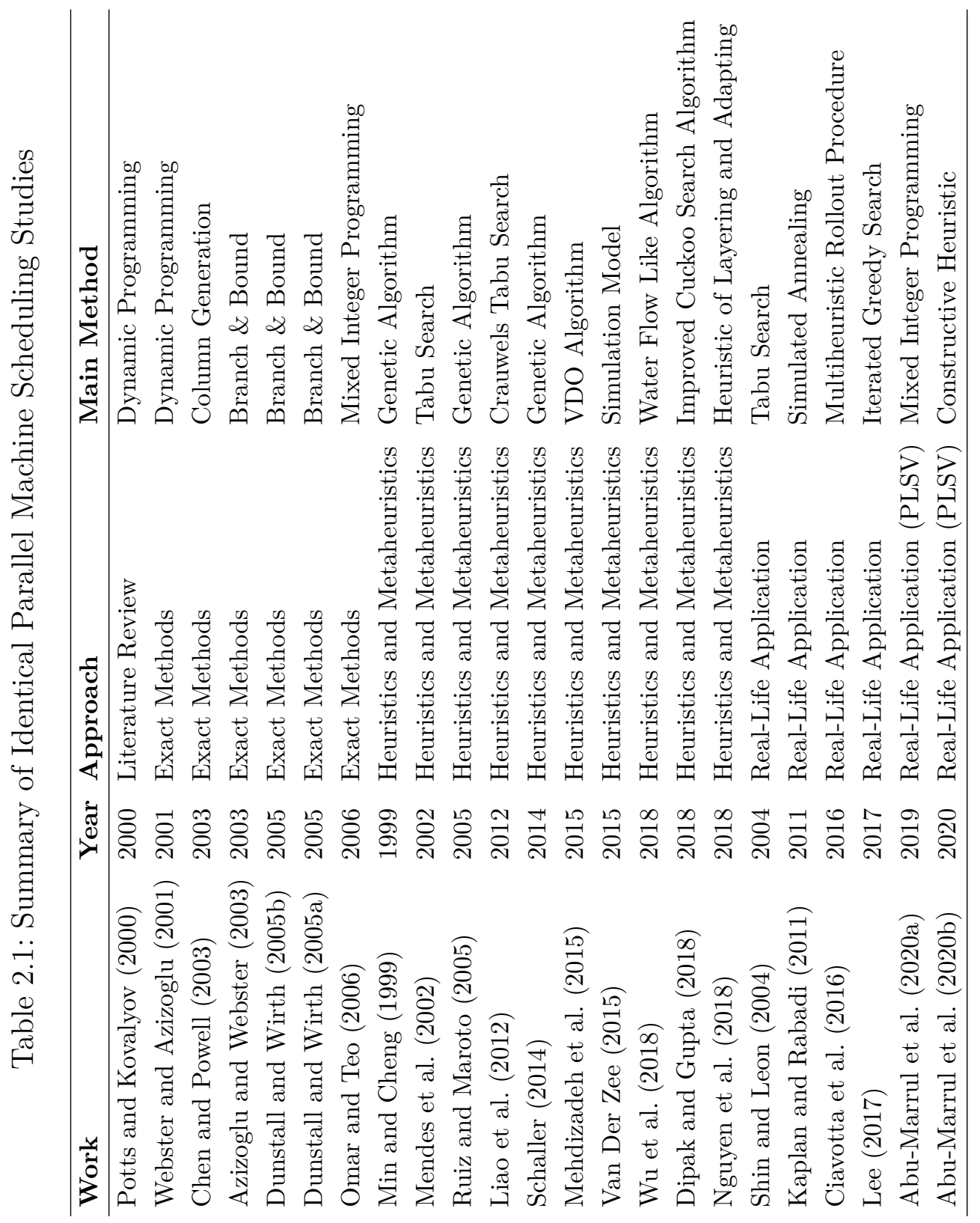




\section{3}

\section{Mathematical Formulation}

\section{1}

\section{Problem Description}

As mentioned in Section 1, the PLSV scheduling problem can be modeled as an identical parallel machine scheduling problem, due to the similar characteristics between them. The vessels are analogous to the machines, which will process the operations in a specific well, which we call job. The operations are processed by the machines and are associated to jobs, but the jobs are not directly processed. When all operations associated to a job is finished, the job is considered complete. In this work we will use machine scheduling notation to describe the problem and the further mathematical formulation.

Let $\mathcal{O}$ be the set of operations, each one belonging to a family of the set $\mathcal{F}$. These operations are scheduled in a set $\mathcal{M}$ of machines, which will process these operations in a particular order. Associated to each family, there is a setup time $s_{f}$, independent from the machine and considered whenever there is a change on the family of operations or when the machine capacity $q_{k}$ is reached. The setup time $s_{f}$ marks the beginning of a voyage, which in machine schedule notation is called a batch. The problem studied is classified as non-preemptive, meaning that operations must be processed at once and cannot be divided. Regarding the operations $i \in \mathcal{O}$, we consider their processing times $p_{i}$ and release dates $r_{i}$. All operations must be associated to at least one job, defined in the set $\mathcal{N}$, meaning that jobs are affected by this operation. One operation can be related to more than one job, indicating the dependence between these jobs. The subset of operations that compose a job $j$ is defined as $\mathcal{O}_{j}$. These jobs have weights $w_{j}$ related to their production rate. To consider a job completed all the associated operations must be finished. On the other way, the subset $\mathcal{N}_{i}$ defines the jobs related to an operation $i$. Each machine has a release date $r_{k}$ that means the date when it is available to operate. We also consider eligibility between machines and operations, denoted by the subset $M_{i}$ indicating which machines can process each operation $i$.

The vessels voyages are considered as batches on the parallel machine 
scheduling problem. They consist on a sequence of operations of the same family preceded by a setup time. This setup time represents the loading of the necessary equipment on the origin port and the navigation time to reach the wells. Due to that, the setup times can only start when all operations of the same batch are released (meaning that all necessary equipment for performing every operation of the voyage has arrived). This particularity is called non-anticipatory setup time. These batches must respect the capacity of the machine $q_{k}$, by summing the load occupation $l_{i}$ of the operations scheduled on this batch.

The objective function of the problem is to minimize the weighted completion time of all jobs, $\sum_{j \in \mathcal{N}} w_{j} C_{j}$, subject to all mentioned constraints. The completion time of each job, $C_{j}$, is calculated by the maximum completion time of all associated operations: $\max _{i \in \mathcal{O}_{j}} C_{i}$. The objective is to finish as soon as possible the most productive jobs.

Regarding the complexity of the PLSV scheduling problem, Mokotoff (2004) prove that the classical identical parallel machine scheduling problem is NP-hard. By simplifying some parameters of the PLSV scheduling problem, we can model it exactly as the classical identical parallel machine scheduling problem. To do that we must set all operations to be eligible to all machines, all operations being associated with a single job, set the families of all operations as 1 and the correspondent setup times as zero and setting the release dates of all operations and machines as the first day of the time horizon. Since we can model the PLSV scheduling problem as the classical identical parallel machine scheduling problem, which we know is NP-hard, and the complete PLSV scheduling problem has even more constraints and parameters, we can conclude that the PLSV scheduling problem is also NP-hard. 


\section{2 \\ Positional Scheduling Formulation}

In this section we present a positional scheduling formulation described by Unlu and Mason (2010) and extended by Abu-Marrul et al. (2020a) for the PLSV problem. There are other possible formulations for this problem, such as the Time-index, the Bucket-index and the Batch Scheduling formulation. We introduce this positional schedule formulation because it fits well the solution representation presented in this study methodology, presented in the next chapter, where the machines are vectors that contains an element (setup or operation) in each position. The positional formulation illustrates the problem's constraints and parameters and inspired the proposed metaheuristic presented in this study.

The positional scheduling formulation consists on representing the machines with a sequence of positions. The model then defines, for each position, if it will allocate an operation or a setup time, respecting all constraints. In our case, the resulting schedule aims to minimize the weighted completion time of the jobs, but it can optimize a different objective function, such as minimize tardiness, maximize production, minimize completion time, among others.

The set $\mathcal{P}_{k}$ indexes the positions of each machine. The decision of allocating an operation or a family setup time is represented by two binary variables: $X_{i k}^{p}$ representing an operation $i$ allocated at the $p$-th operation of the machine $k$, and $Y_{f k}^{p}$ representing a setup time on the $p$-th position of machine $k$ of the family $f$.

To keep track of some critical information during the model's decisions the authors define three other continuous variables, $S_{k}^{p}$ defines the start of each position $p$ in machine $k$ and $C_{i}$ and $C_{j}$, representing the completion time of operations and jobs, respectively.

To deal with the occupation constraint, the continuous variables $L_{k}^{p}$ are added representing the cumulative load occupancy in position $p$. To treat the non-anticipatory setup times, the variables $R_{k}^{p}$ represent the release time of the position $p$, to guarantee that the start of positions which have setup times allocated will respect the release dates of all operations on the batch.

The positional scheduling formulation is then described bellow:

$$
\min \sum_{j \in \mathcal{N}} w_{j} C_{j}
$$

subject to 


$$
\begin{aligned}
& \sum_{k \in \mathcal{M}_{i}} \sum_{p \in \mathcal{P}_{k}} X_{i k}^{p}=1 \quad \forall i \in \mathcal{O} \\
& \sum_{i \in \mathcal{N}} X_{i k}^{p}+\sum_{f \in \mathcal{F}} Y_{f k}^{p} \leq 1 \quad \forall k \in \mathcal{M}, p \in \mathcal{P}_{k} \\
& L_{k}^{p} \geq L_{k}^{(p-1)}+\sum_{i \in \mathcal{O}} l_{i} X_{i k}^{p}-\sum_{f \in \mathcal{F}} q_{k} Y_{f k}^{p} \quad \forall k \in \mathcal{M}, p \in \mathcal{P}_{k} \\
& L_{k}^{p} \leq q_{k} \\
& \sum_{i \in \mathcal{N}_{f}} X_{i k}^{p} \leq \sum_{i \in \mathcal{N}_{f}} X_{i k}^{(p-1)}+Y_{f k}^{(p-1)} \\
& \forall k \in \mathcal{M}, p \in \mathcal{P}_{k} \\
& \forall k \in \mathcal{M}, p \in \mathcal{P}_{k}, f \in \mathcal{F} \\
& R_{k}^{p} \geq \sum_{i \in \mathcal{O}} r_{i} X_{i k}^{(p+1)} \quad \forall k \in \mathcal{M}, p \in \mathcal{P}_{k} \\
& R_{k}^{p} \geq R_{k}^{(p+1)}-\sum_{f \in \mathcal{F}} r_{\text {max }} Y_{f k}^{(p+1)} \quad \forall k \in \mathcal{M}, p \in \mathcal{P}_{k} \\
& S_{k}^{p} \geq S_{k}^{(p-1)}+\sum_{i \in \mathcal{O}} p_{i} X_{i k}^{(p-1)}+\sum_{f \in \mathcal{F}} s_{f} Y_{f k}^{(p-1)} \quad \forall k \in \mathcal{M}, p \in \mathcal{P}_{k} \\
& S_{k}^{p} \geq r_{k} \quad \forall k \in \mathcal{M}, p \in \mathcal{P}_{k} \\
& S_{k}^{p} \geq R_{k}^{p} \quad \forall k \in \mathcal{M}, p \in \mathcal{P}_{k} \\
& C_{i} \geq S_{k}^{p}+p_{i}-\left(1-X_{i k}^{p}\right) M \quad \forall i \in \mathcal{O}, k \in \mathcal{M}, p \in \mathcal{P}_{k} \\
& C_{j} \geq C_{i} \\
& \forall j \in \mathcal{N}, i \in \mathcal{O}_{j} \\
& X_{i k}^{p} \in\{0,1\} \\
& Y_{f k}^{p} \in\{0,1\} \\
& \forall i \in \mathcal{O}, k \in \mathcal{M}_{i}, p \in \mathcal{P}_{k} \\
& \forall k \in \mathcal{M}, p \in \mathcal{P}_{k}, f \in \mathcal{F} \\
& \forall i \in \mathcal{O} \\
& \forall k \in \mathcal{M}, p \in \mathcal{P}_{k}
\end{aligned}
$$

The Objective Function (3-1) is to minimize the weighted completion time of the jobs. Constraints (3-2) guarantee that every operation will be allocated once in the schedule. Constraints (3-3) ensure that we schedule a setup time or an operation in each position, not both. Constraints (3-4) keep track of the occupation load in each position, summing the occupancy load when an operation is allocated in the position and setting the variable to zero when it is a setup. Constraints (3-5) ensure that the machine occupancy capacity is respected. Constraints (3-6) forbid an operation of one family to be scheduled after an operation or setup time of a different family. Constraints (3-7) calculate the release of a position based on the release date of the next operation allocated on that machine. Constraints (3-8) guarantee that the release of a position is greater or equal than the release variable of the next position. These constraints (3-7) and (3-8) together ensure that the non-anticipatory setup times will be respected. Constraints (3-9) compute the start time of a position 
by summing the start time of the precedent position with the processing time of the scheduled operation or setup time allocated. Constraints (3-10) forbid the start time of a position in a machine to be lower than the release date of the machine. Constraints (3-11) guarantee that a position can only start after it is released. Constraints (3-12) compute the completion time of operations by summing the start time of the position with the processing time of the allocated operation. Constraints (3-13) force the completion time of a job to be the last completion time of all operations associated to that job. Constraints (3-14)-(3-17) are the non-negativity and domain constraints. 


\section{4}

\section{Methodology}

The first aspect of this work methodology is the solution representation. Based on it, the methods are developed aiming to minimize the weighted completion time of jobs. The next step of the methodology is obtaining an initial solution for the identical parallel machine scheduling problem using a constructive heuristic. After that, we define the neighborhoods that will be explored in our search: Relocate, Swap, Setup Insertion and Setup Removal. With these neighborhoods defined, we propose a Local Search procedure. At last, we present the Iterated Local Search metaheuristic that looks for high quality solutions for the PLSV Scheduling Problem. The next sections describe in detail each one of these steps.

\section{1}

\section{Solution Representation And Evaluation}

The positional formulation introduced inspired our solution representation for this work. In the mathematical formulation, the machines can be represented as vectors. For each machine, there are $n$ positions containing an operation or a setup time. Representing the solutions as vectors of integers saves computational time when performing movements within the solution, but neglects the starting time information for each operation and setup time, which are used to calculate our objective function of minimizing the weighted completion time. To deal with this problem, we created an evaluation function that receives the vectors of integers and calculates the minimum starting time for each position respecting all mentioned constraints. The evaluation function runs in linear time in the number of operations. In Figure 4.1, the solution representation is exposed and then the solution representation indicating the starting times for each element. 


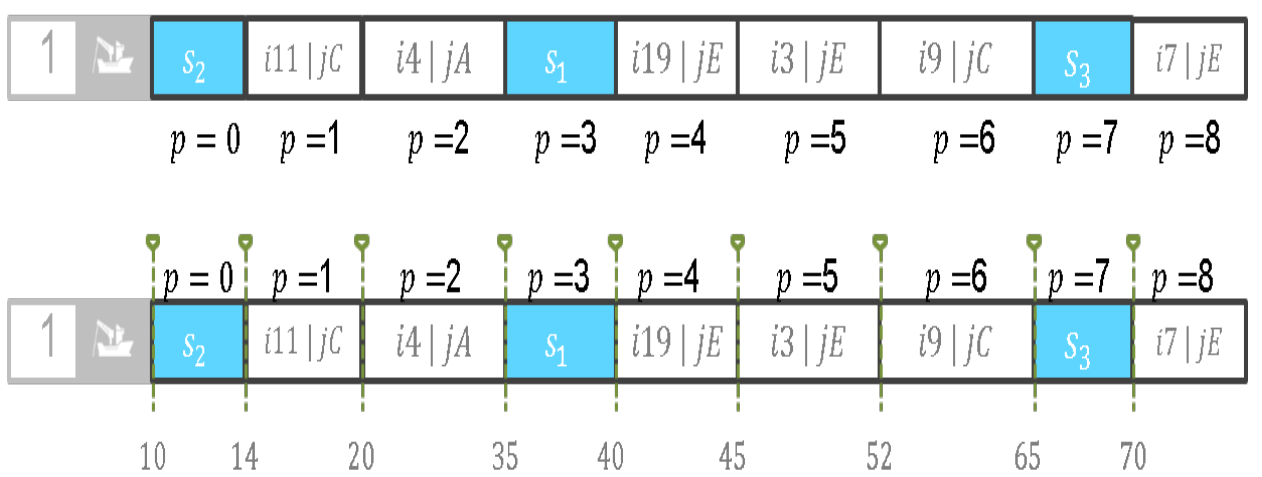

Figure 4.1: Solution Representation and Starting Times

\section{2}

\section{Constructive Heuristic}

In this section, we present the Schedule Construction Procedure, proposed by Abu-Marrul et al. (2020b), to provide an initial solution for our problem. Since it is an important part of this study methodology we will explain in detail the approach.

The Schedule Construction Procedure decides at each iteration, for an operation, in which machine it will be allocated and if it is allocated in an existing batch or a new one. The authors define a set that at first contains all operations. To define the order of operations to be allocated, a set of dispatching rules to rank all operations is considered. These dispatching rules will be explained further in subsection 4.2.1. If two operations are tied on the dispatching rule criteria, the algorithm chooses the one with the lower index. To decide in which machine the operation is allocated, the machines' completion times are evaluated. The operation will be performed by the machine that has the lower machine completion time including the recently allocated operation, respecting the eligibility constraints and the machines release dates. To decide if the operation will enter in an existing batch or a new one, the family and capacity constraints are checked. After the operation is allocated, the method removes it from the set of unscheduled operations. The algorithm runs until all operations are scheduled. It is summarized in Algorithm 1. 


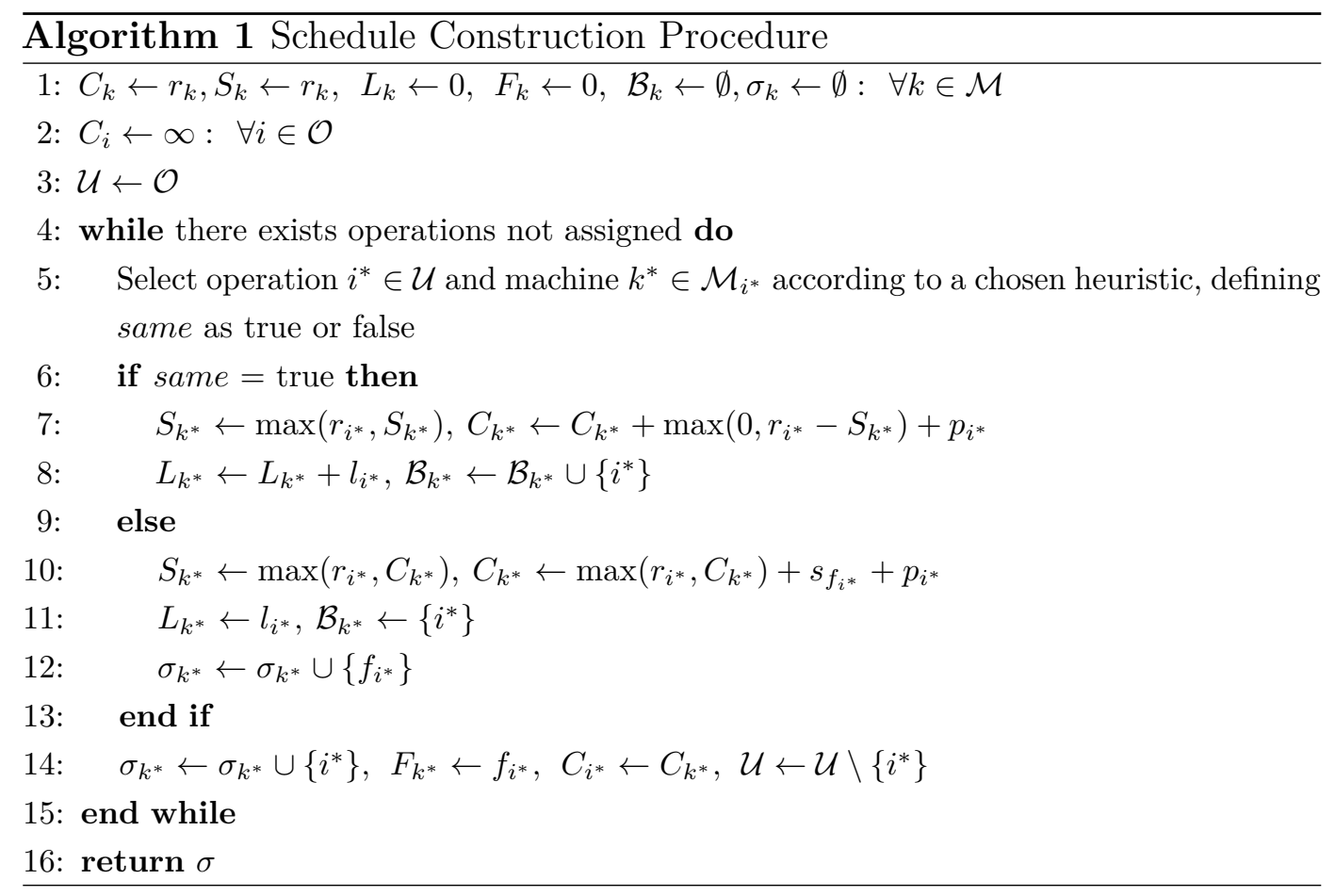

The Schedule Construction Procedure first sets the initial values for the variables: completion time of each machine, $C_{k}$, and starting time for the current batch for each machine, $S_{k}$, as the release date of that machine, $r_{k}$. The loading occupancy of the current batch for each machine, $L_{k}$, the family of the current batch in the machine, $F_{k}$, the set of operations scheduled in the current batch in the machine, $B_{k}$, and the list of schedules for each machine, $\sigma_{k}$, are set as zero. While there are unscheduled operations, the method selects one operation and machine, based on a specific dispatching rule. Then the algorithm checks if it is feasible to schedule it in an existing batch. If it is, the operation is inserted in this batch, the variables that control the starting time of the batch in the machine, completion time of the machine, load occupancy capacity of the machine, and operations scheduled in the batch in the machine are updated. Otherwise, the operation is scheduled in a new batch, including it's respective setup time. The variables that control the starting time of the batch in the machine, completion time of the machine, load occupancy of the batch in the machine, operations scheduled in the batch in the machine and list of schedules in the machine are updated. The algorithm stops when all operations are scheduled.

An illustrative scheme of the Schedule Construction Procedure is exposed in Figure 4.2. We will illustrate the Schedule Construction Procedure in a small example, using the Earliest Release Date (ERD) dispatching rule to explain how the method construct batches. The ERD rule consists on ordering all operations by the release date criteria. The operations that have earlier release 
dates are scheduled first. The operations and machines data are presented in Tables 4.1 and 4.2 ordered by their respective release dates.

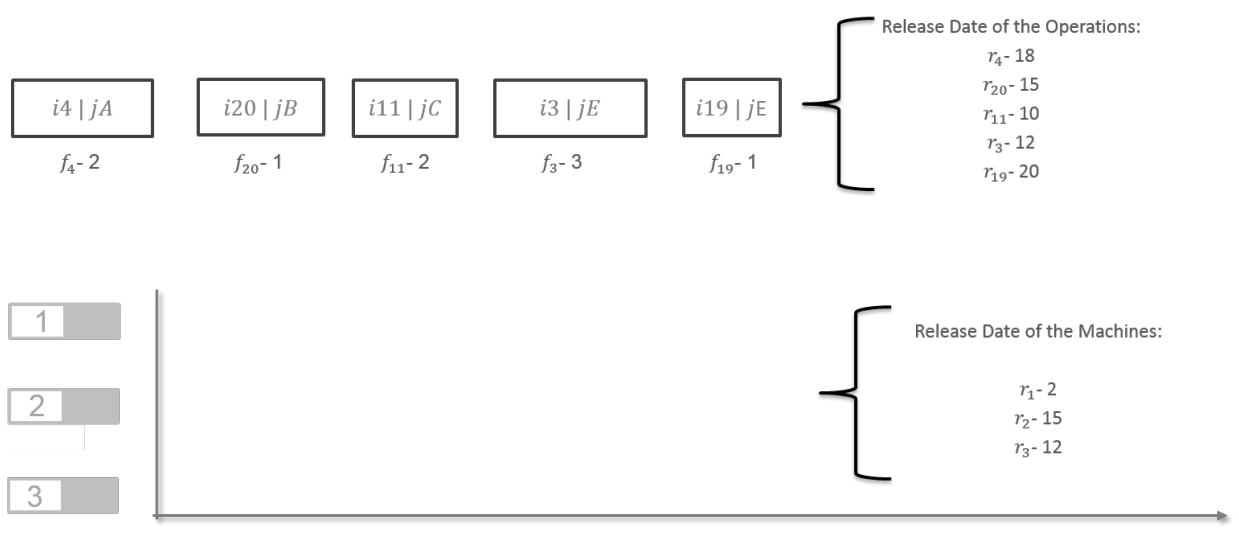

Figure 4.2: Schedule Construction Procedure Scheme

Source: The Author

Table 4.1: Operations Data

\begin{tabular}{ccc}
\hline $\mathcal{O}$ & Family & Release Date \\
\hline$i_{11}$ & 2 & 10 \\
$i_{3}$ & 3 & 12 \\
$i_{20}$ & 1 & 15 \\
$i_{4}$ & 2 & 18 \\
$i_{19}$ & 1 & 20 \\
\hline
\end{tabular}

Table 4.2: Machines Data

\begin{tabular}{cc}
\hline $\mathcal{M}$ & Release Date \\
\hline$k_{1}$ & 2 \\
$k_{3}$ & 12 \\
$k_{2}$ & 15 \\
\hline
\end{tabular}

In this illustrative example, we have five operations, belonging to four jobs and three different families. For example $i_{4} \mid j A$ means that operation $i_{4}$ is associated to the job $j A$ and the family of an operation is expressed as $f_{i}$. Each of these operations and machines have a specific release date. To simplify, in this example we consider that all operations are eligible at all machines and we do not consider occupation constraints, but in the real problem it is not possible to allocate operations in machines that are not eligible to perform them or create batches that surpasses the machine capacity. The algorithm starts by selecting the first operation to be allocated. To do that the method uses one of the dispatching rules proposed. In this illustrative example, we are using the ERD dispatching rule to decide in each order the operations will be 
allocated. In spite of not being the best dispatching rule in terms of results, we decided to use it in the example because it is intuitive to understand how it ranks the operations, by simply selecting the operations with lower release dates first. The Schedule Construction Procedure is exactly the same regardless of the dispatching rule used to prioritize the operations, the only difference between them is the order of allocation. Once the operation is chosen, the algorithm allocates it in the machine that will give the minimum completion time. The procedure selects the operation $i_{11}$, which is the first to be released at day 10 and allocate it at machine 1 because it is released on day 2 , as the other machines are not released yet in day 10. The first allocation is presented in Figure 4.3.
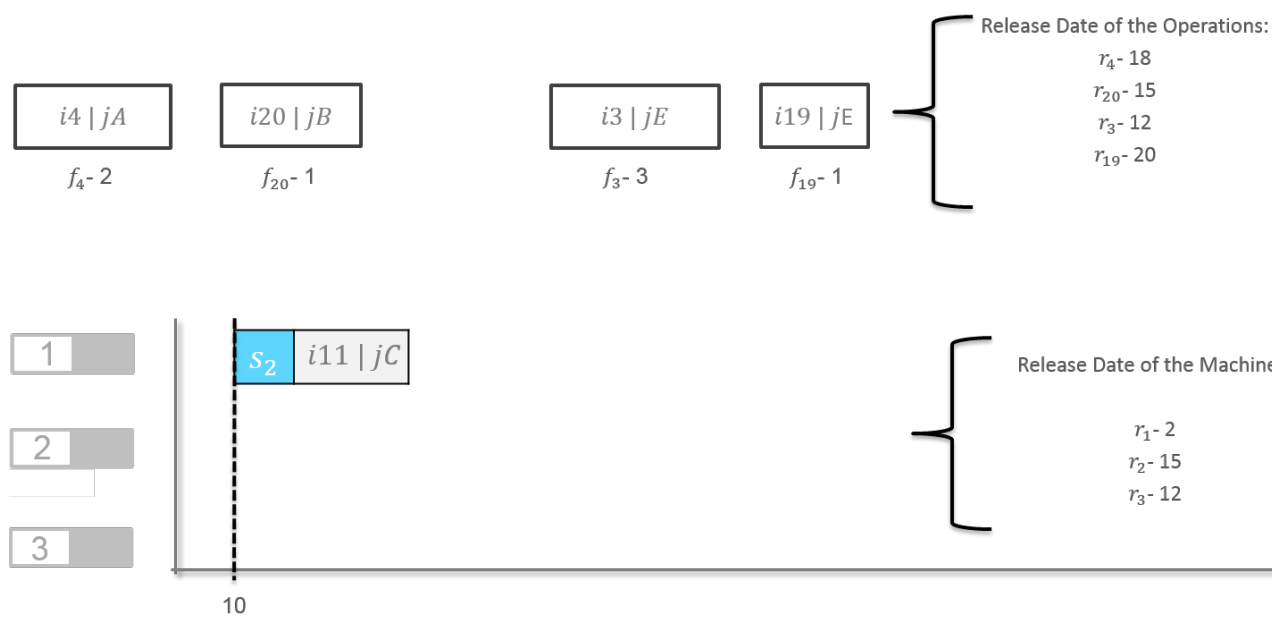

Figure 4.3: First Allocation on Schedule Construction Procedure

Source: The Author

Following the ERD rule, the second operation to be scheduled is the operation $i_{3}$, which is released on day 12 . It can be allocated in machine 1 creating a new batch after the end of operation $i_{11}$, because it is from a different family, in machine 2 starting on day 15, when the machine will be released, or in machine 3 starting on day 12. As the algorithm aims to allocate it in the machine that will have the lowest completion time, it is allocated in machine 3 , as illustrated in Figure 4.4.

The next operation to be allocated following the ERD rule is $i_{20}$. It can be scheduled in machine 1 , but since it is from a different family than $i_{11}$, it will create a new batch after it. The same will happen in machine 3 , where it will create a new batch after operation $i_{13}$. Since machine 2 is released on day 15 we can allocate operation $i_{20}$ and have the minimum machine completion time, as we can see in Figure 4.5. 

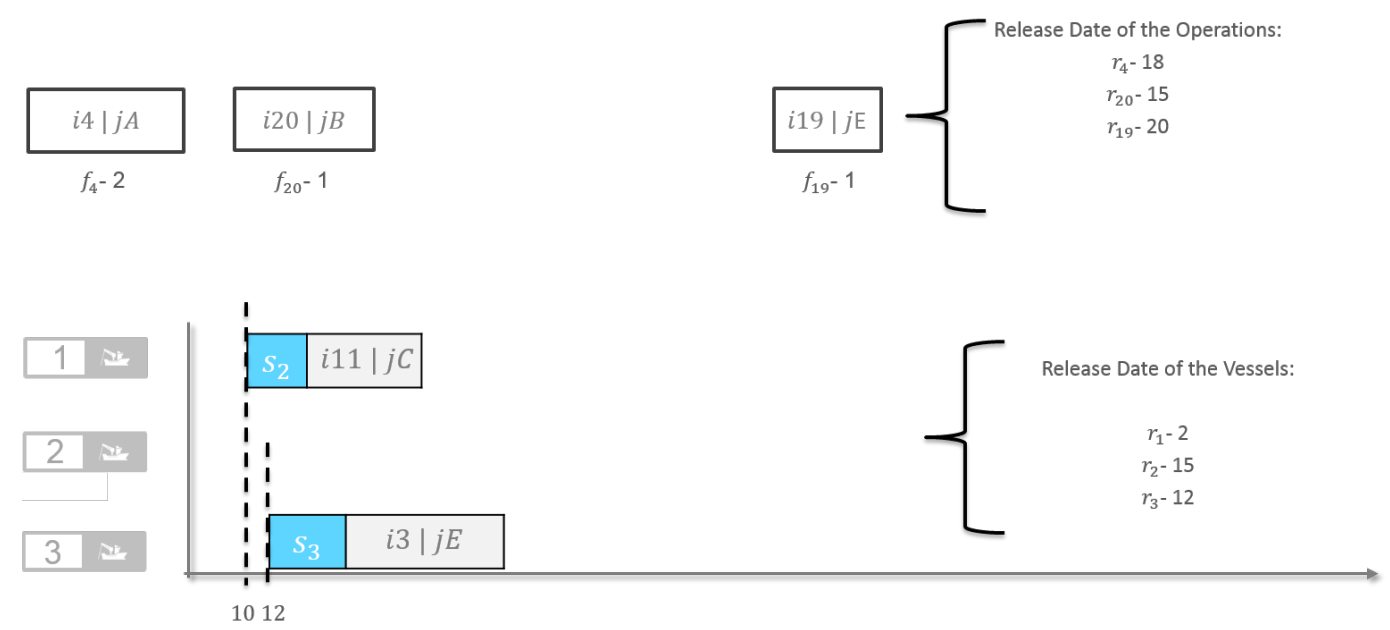

Figure 4.4: Second Allocation on Schedule Construction Procedure Source: The Author
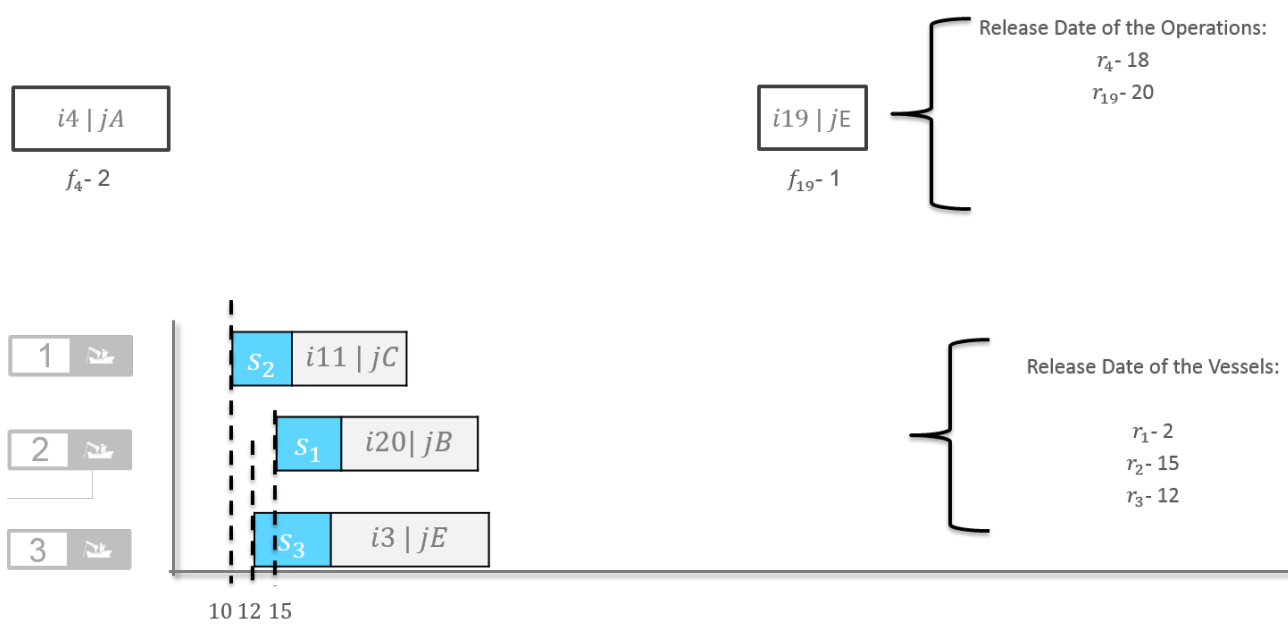

Figure 4.5: Third Allocation on Schedule Construction Procedure

Source: The Author

Observing the release date of the remaining operations, we notice that the next one to be scheduled is operation $i_{4}$. This operation is from family 2 , so it can be allocated in machine 2 or 3 , creating new batches, or in machine 1 in the same batch of operation $i_{11}$. One important characteristic of this step is that by adding this operation to the same batch on machine 1 we need to shift all batch forward due to the non-anticipatory setup times. Evaluating the completion time of machines, we see that this is the best allocation in this scenario, moving the setup of this batch from day 10 to day 18, as observed in Figure 4.6.

To allocate the last operation, the same logic is applied. The operation $i_{19}$ is from family 1 , the same of operation $i_{20}$. So it can be allocated in the same batch on machine 2 or in new batches on machines 1 and 3. Scheduling it in 

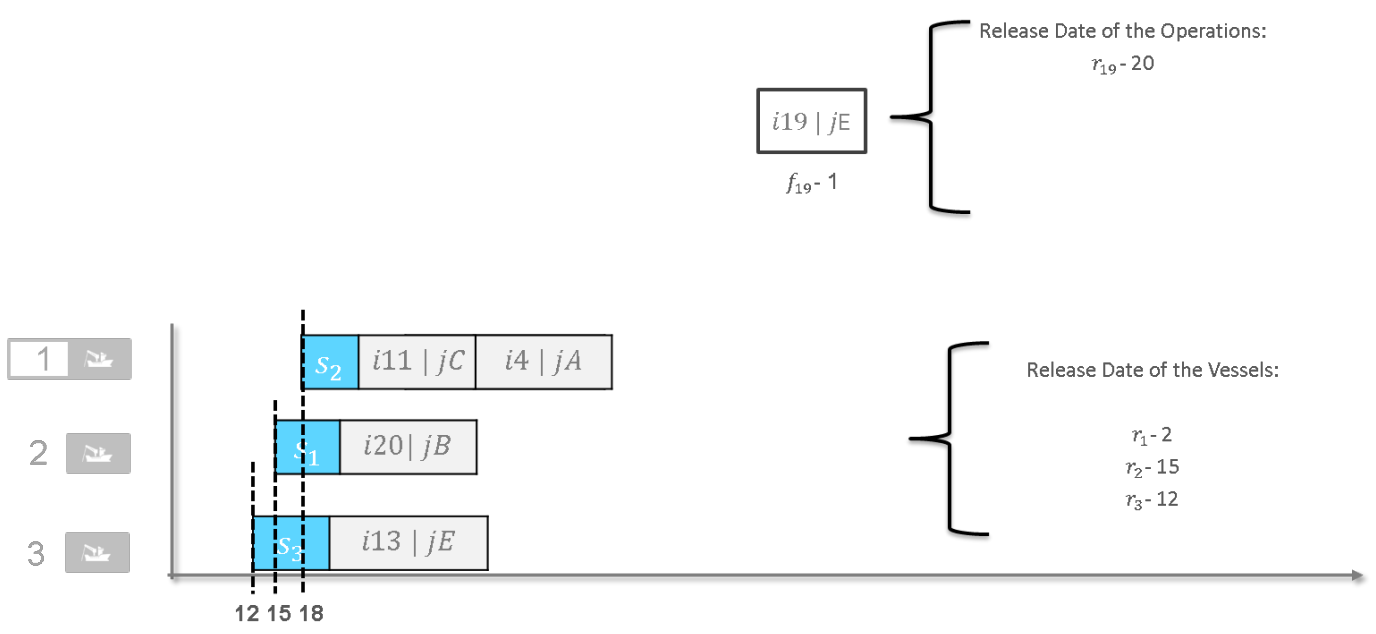

Figure 4.6: Fourth Allocation on Schedule Construction Procedure

Source: The Author

machine 2 will shift the whole batch to start at the release date of operation $i_{19}$, day 20, because of the non-anticipatory setup times. Considering the machine completion time, this is the best way to allocate operation $i_{19}$, represented on Figure 4.7. Since all operations are allocated, we can consider that we have an initial solution for this illustrative problem. To evaluate this solution we calculate the weighted completion time of jobs as described on Section 3.1. This procedure is taken aiming to provide an initial solution as input to the local search procedures and metaheuristic that will follow on the study.
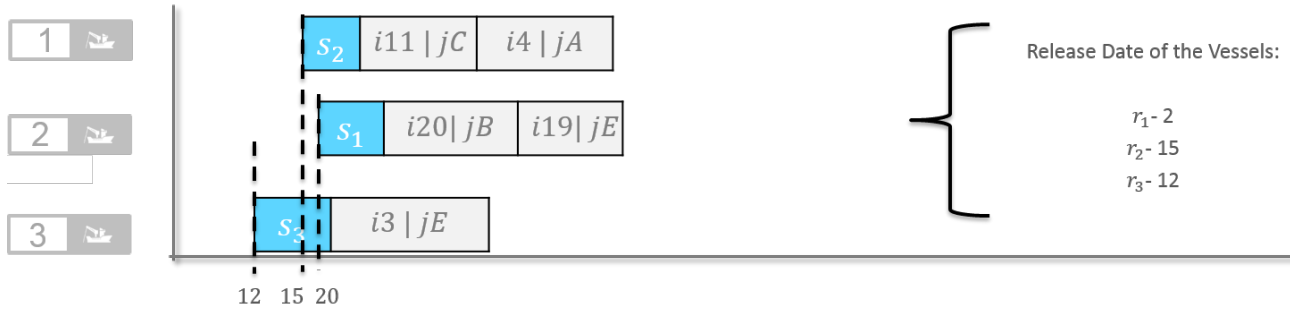

Figure 4.7: Fifth Allocation on Schedule Construction Procedure

Source: The Author

\subsection{1}

\section{Constructive Heuristic Decision}

To decide in which order the operations will be allocated, Abu-Marrul et al. (2020b) define a set of dispatching rules. For each instance, we run the Constructive Heuristic with all dispatching rules and select the five with the minimum weighted completion time. One important characteristic of our 
problem is that our instances only consider weights of jobs, but in the Weighted Shortest Processing Time (WSPT) and Weighted Minimum Completion Time (WMCT) dispatching rules the weights of operations are considered. In the WSPT rule, the operations are ranked by their weight divided by their processing times, while the WMCT rule ranks the operations by their minimum possible completion time divided by their weights. To deal with that that the authors propose five different techniques to estimate the weights of operations:

- MAX: Maximum weight among associated jobs, computed as $w_{i}=$ $\max _{j \in \mathcal{N}_{i}} w_{j}$

- SUM: Sum of the weights among associated jobs, computed as $w_{i}=$ $\sum_{j \in \mathcal{N}_{i}} w_{j}$

- AVG: Average weight among associated jobs, computed as $w_{i}=$ $\sum_{j \in \mathcal{N}_{i}} w_{j} /\left|\mathcal{N}_{i}\right|$

- WAVG: Weighted average weight among associated jobs, computed as $w_{i}=\sum_{j \in \mathcal{N}_{i}} w_{j} /\left|\mathcal{O}_{j}\right|$

- WAVGA: WAVG adjusted at each iteration by the set of non-scheduled operations.

The Weighted Adaptive Average (WAVGA) strategy divides the weight of the jobs for each associated operation. When one of these operations is scheduled, its estimated weight is equally distributed by all unscheduled operations of that job, raising their weight. That strategy manipulates the method to select the unscheduled operations associated to a job when most of its associated operations are already scheduled.

The authors tested six dispatching rules as candidates to rank the operations in the Constructive Heuristic. The priority value $\pi_{i}$ is the comparison criteria to decide the next operation to schedule. At each iteration, the operation $i$ with the largest $\pi_{i}$ is selected. $T_{i}$ is defined as the minimum possible completion time among the eligible machines for each operation $i$, and is computed as $T_{i}=\min _{k \in \mathcal{M}_{i}} C_{k}$. The other parameters included in the dispatching rules are defined in Chapter 3. As the original dispatching rules do not consider family setup times or batches, the authors adapted the rules including these parameters. The following dispatching rules are considered:

- ERD: Earliest Release Date $\pi_{i}=1 / r_{i}$.

- SPT: Shortest Processing Time $\pi_{i}=1 / p_{i}$.

- LPT: Longest Processing Time $\pi_{i}=p_{i}$.

- MCT: Minimum Completion Time $\pi_{i}=\max \left(T_{i}, r_{i}\right)+p_{i}+s_{f i}$. 
- WSPT: Weighted Shortest Processing Time $\pi_{i}=w_{i} / p_{i}$.

- WMCT: Weighted Minimum Completion Time $\pi_{i}=\left[\max \left(T_{i}, r_{i}\right)+p_{i}+\right.$ $\left.s_{f i}\right] / w_{i}$.

We run the Schedule Construction Procedure with all dispatching rules and all weight estimators for each dispatching rule that consider operations weights. After that, we store the five best different solutions. This solutions will serve as input for the multi-start strategy in our further Iterated Local Search.

\section{3}

\section{Neighborhoods}

After finding an initial solution for the problem via the Schedule Construction Procedure we need to define the Local Search neighborhood structures. These procedures define how the algorithm will perform movements in the current solution in order to improve it. The next subsections describe the four neighborhoods that will guide our further Local Search. Before explaining them, we will introduce the erase and insert functions that are the foundation of the neighborhoods movements, since all of them consist on inserting and/or erasing elements in the schedule. Both movements take constant time plus one evaluation of the schedule, then we can conclude that they run in linear time.

\subsection{1}

\section{Erase Function}

The erase function defines the complete set of rules that must be respected when erasing a position in the schedule. The function depends of the element contained in the position that we want to erase. We use the index $p$ to define the position in the vector which is equivalent to a position in a machine schedule (operation or setup). Here we will use the notation of operations family $O_{f}$, without indicating the job associated to the operation because in the erase/insertion analysis it is not relevant and would pollute the illustrations. We just present the family of each operation and setup time because that is the main information to define how the insertion and removal of elements will proceed. There are two possible cases where the erase function applies: if $p$ is an operation in a single-operation batch or if $p$ is an operation in a multiple-operations batch. If $p$ is a setup time, the element is not erased.

Furthermore, if position $p$ is an operation in a single-operation batch, the function erases the operation in position $p$ and also the setup in position $p-1$. 
It occurs because it would generate a batch containing just a setup time, which is not allowed in our problem. An illustration of this case is exposed in Figure 4.8 .
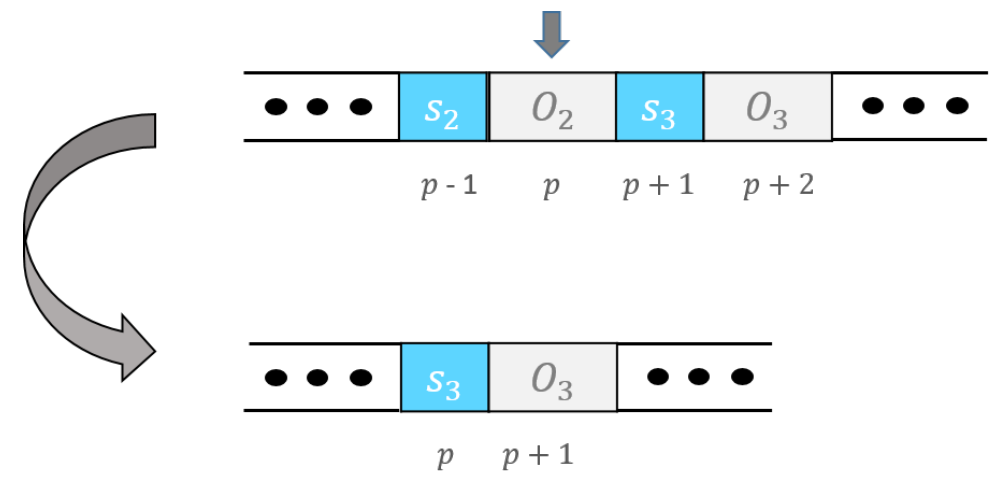

Figure 4.8: Erase Function - Single Operation Batch

Source: The Author

If $p$ is an operation in a multiple-operations batch the erase function just delete this operation. It occurs because the removal of the operation does not result in an invalid batch composition (when a batch does not contain a setup preceding an operation). This behavior is explained in Figure 4.9.

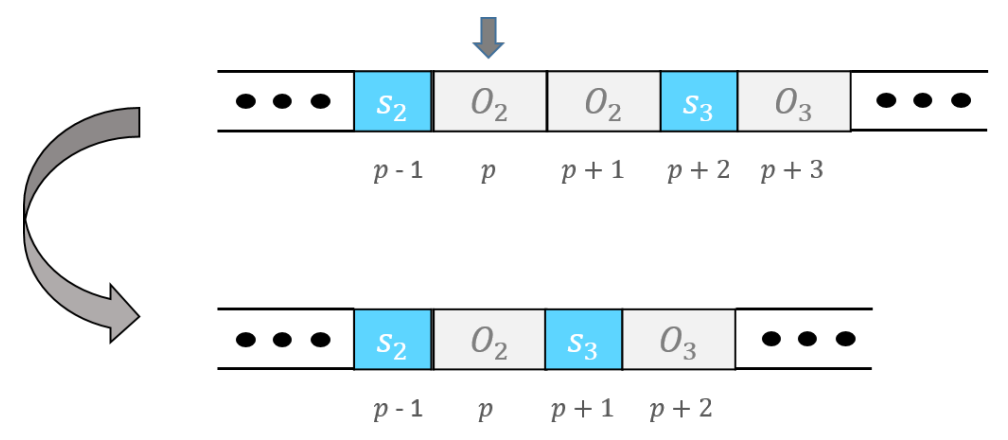

Figure 4.9: Erase Function - Multiple Operation Batch

Source: The Author

\subsection{2}

\section{Insert Function}

The insert function defines the complete set of rules to insert one operation in our schedule. Depending on the operation that will be inserted and its target position, the function has different outcomes. The procedure consists on inserting a predefined operation, from an specific family, in the schedule. There are five possible cases depending on the position where the operation 
will be inserted. In the illustrative examples we assume that we are inserting an operation from family 3 in a different position in the schedule, denoted $O_{3}$. We use the index $p$ to define the position in the vector which is equivalent to a position in a machine schedule (operation or setup).

- The first case is when the position $p$ is the first position of the schedule in the machine. In this case, we insert the setup time of the same family as the operation that will be inserted in position $p$ and the operation in position $p+1$. An illustrative example is shown in Figure 4.10.
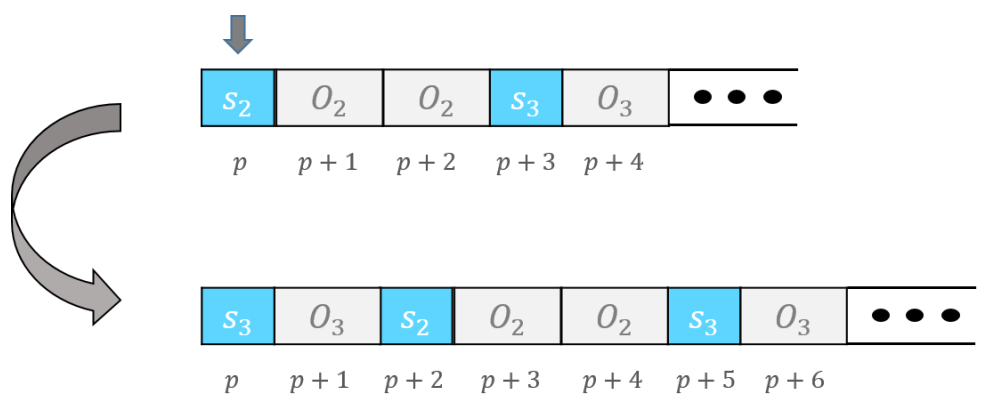

Figure 4.10: Insert Function - case 1

Source: The Author

- The second case is when position $p-1$ is a setup of the same family of the operation to be inserted. In this case, we insert the operation in position $p$. An illustrative example is shown in Figure 4.11.
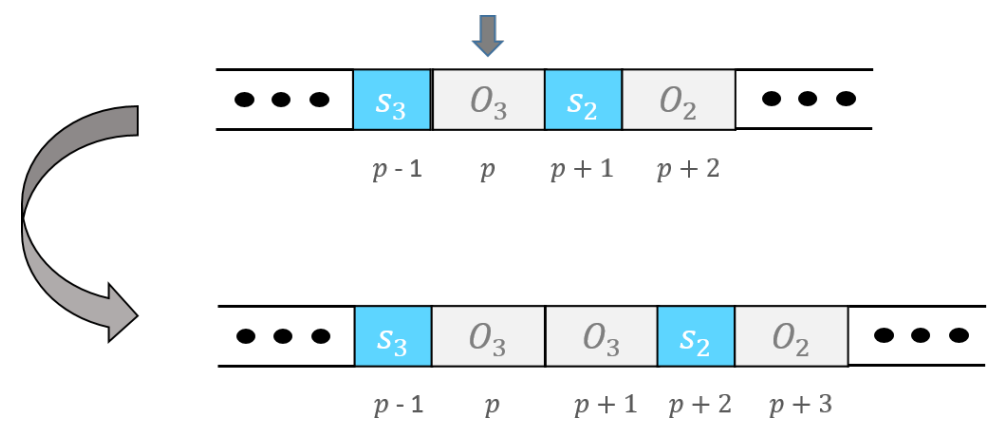

Figure 4.11: Insert Function - case 2

Source: The Author

- The third case is when position $p-1$ is an operation of a family different from the operation that will be inserted, and position $p$ is a setup. In this case, we insert the setup of the same family as the operation to be inserted in position $p$ and the operation in position $p+1$. An illustrative example is shown in Figure 4.12.

- The forth case is when position $p-1$ is an operation of a family different from the operation that will be inserted and position $p$ is also an operation. 


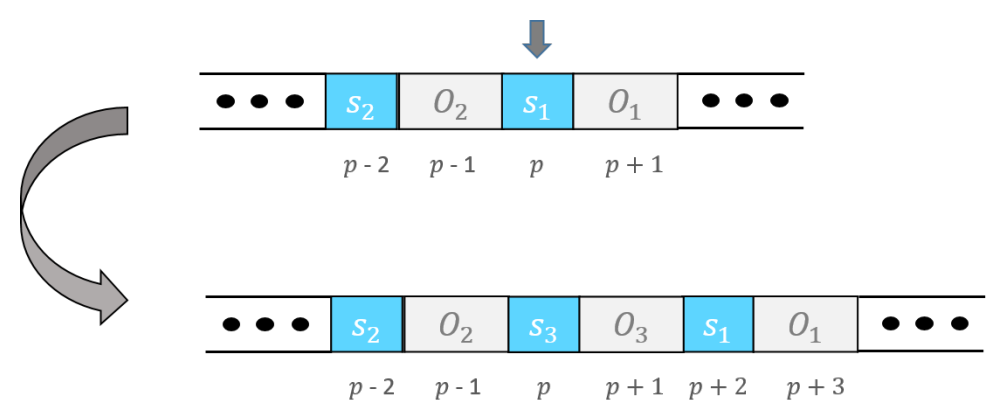

Figure 4.12: Insert Function - case 3

Source: The Author

In this case, we insert the setup of the same family as the operation to be inserted in position $p$, the operation in position $p+1$ and the setup of the family from the operation in $p+2$. An illustrative example is shown in Figure 4.13.

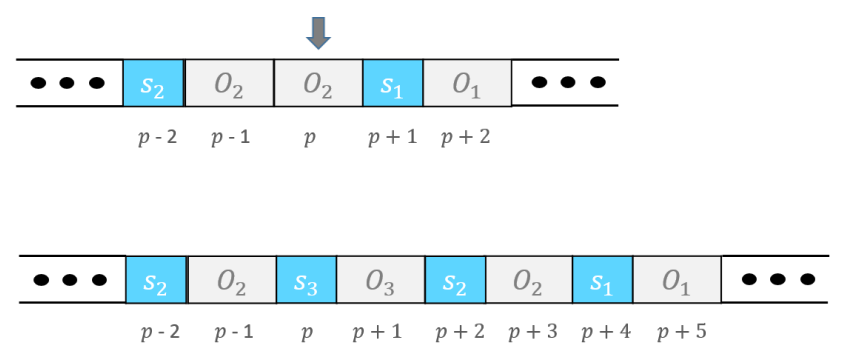

Figure 4.13: Insert Function - case 4

Source: The Author

- The fifth case is when position $p-1$ is a setup from a family different from the operation that will be inserted. In this case we insert the setup of the same family as the operation that will be inserted in position $p-1$ and the operation in position $p$. An illustrative example is shown in Figure 4.14 .

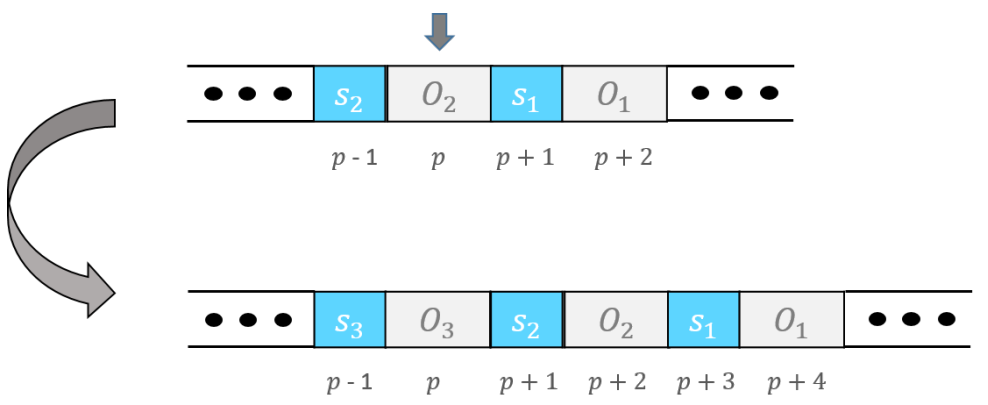

Figure 4.14: Insert Function - case 5

Source: The Author 


\subsection{3 \\ Relocate Neighborhood}

Once initial solutions are obtained by the Schedule Construction Procedure, it is possible to perform movements within these schedules to reach new solutions. The first neighborhood that we explore is the Relocate. The procedure consists on swiping the machine's positions, selecting each operation (one per iteration), and trying to insert it in a different position (in the same machine or in a different one). Depending on which position the operation is moved to, we have to insert one or more setup times in different positions to respect the family constraints.

To explain this method, we describe two different steps of the algorithm: the removal of setups/operations and the insertion of setups/operations using the erase and insert functions explained in sections 4.3.1 and 4.3.2.

The Relocate neighborhood arguments are: the machine and position of the operation to be relocated in the current schedule $\left(m_{1}, p_{1}\right)$ and the machine and position where this operation will be inserted $\left(m_{2}, p_{2}\right)$. The procedure consists in using the erase function in position $p_{1}$ of machine $m_{1}$, returning the number of elements erased. Then it inserts the operation in machine $m_{2}$ using the insert function and evaluates: if machine $m_{1}$ is different from machine $m_{2}$ or position $p_{1}$ is higher than position $p_{2}$, the operation is inserted in position $p_{2}$. Otherwise, the operation is inserted in position $p_{2}$ minus the number of elements erased by the erase function. This happens because as we erase elements in our schedule, the indexes change, moving the position $p$ backwards. An illustrative example of each case is exposed in Figures 4.15 and 4.16.

\subsection{4}

\section{Swap Neighborhood}

The other neighborhood proposed in this work is the Swap. It consists on swiping the machines, selecting two operation per iteration, and changing their positions. Depending on the families of the selected operations more than one element (setup times or operations) need to be erased or inserted.

The Swap neighborhood arguments are: the machine and position of the operation to be swapped $\left(m_{1}, p_{1}\right)$, which we will call operation 1 , and the machine and position of the other operation to be swapped $\left(m_{2}, p_{2}\right)$, which we will call operation 2 . The procedure starts by erasing these operations using the erase function. Then it inserts operation 2 in the machine $m_{1}$ at position $p_{1}$ minus (the number of elements erased when erasing operation 1 ) 


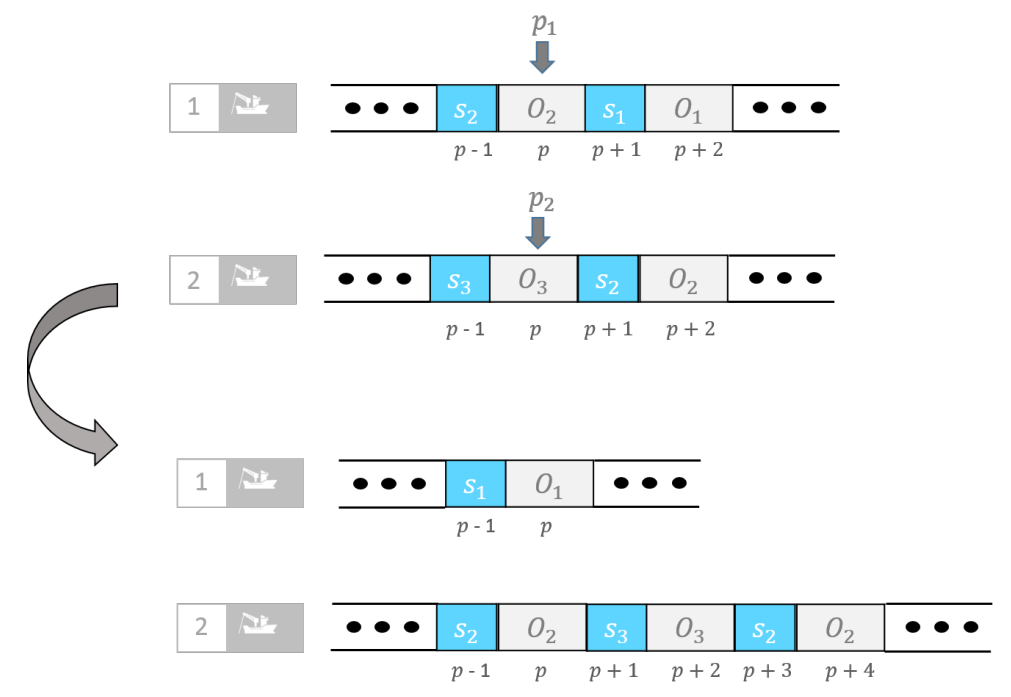

Figure 4.15: Relocate Neighborhood - case 1

Source: The Author

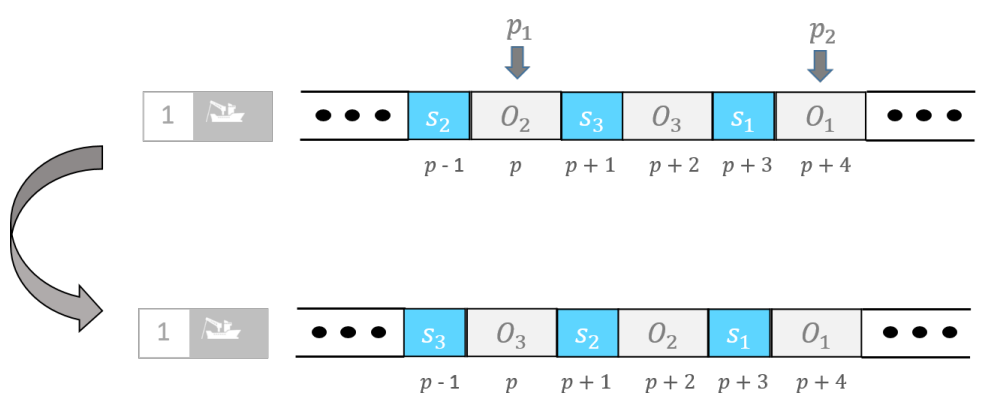

Figure 4.16: Relocate Neighborhood - case 2

Source: The Author

minus 1. Operation 1 is inserted in machine 2, but there are two possible positions for it to be inserted: if machine $m_{1}$ is different than machine $m_{2}$, this operation is inserted in position $p_{2}$ minus (the number of elements erased when erasing operation 2) plus 1 . If machine $m_{1}$ is the same as machine $m_{2}$, this operation is inserted in position $p_{2}$ minus (the number of elements erased when erasing operation 2) plus 1 plus (the number of elements inserted when inserting operation 2 in machine $m_{1}$ ) - (the number of elements erased when erasing operation 1). Again, these particular cases are explained because when erasing or inserting elements, we change the indexes, and the position $p$ can go backwards or forward. An illustrative example of each case is exposed in Figures 4.17 and 4.18 . 


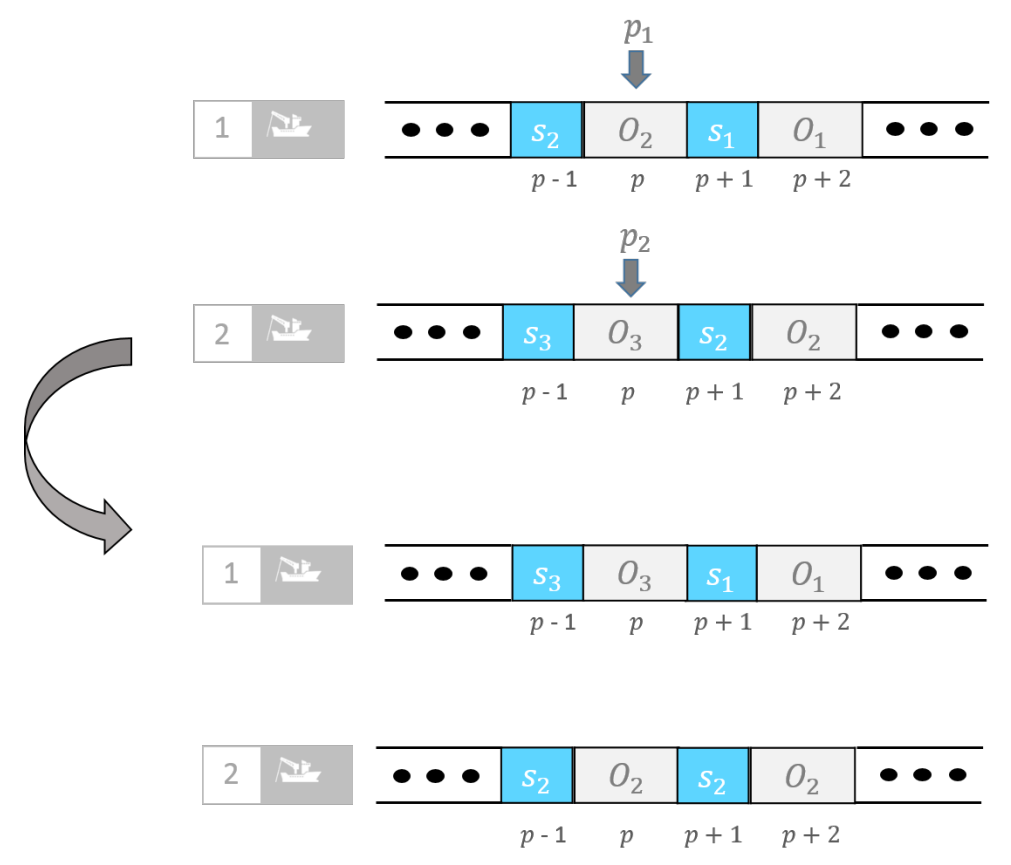

Figure 4.17: Swap Neighborhood - case 1

Source: The Author

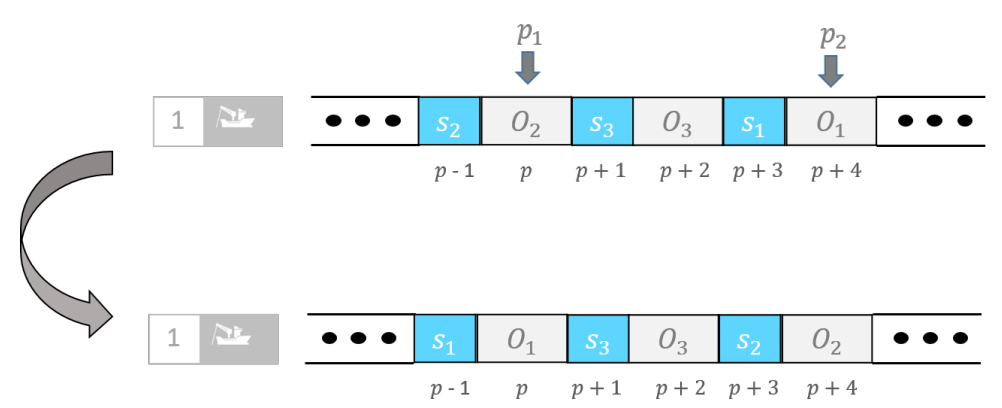

Figure 4.18: Swap Neighborhood - case 2

Source: The Author

\subsection{5}

\section{Setup Insertion and Setup Removal Neighborhoods}

Besides the two neighborhoods explained in the previous sections, we defined new strategies to fully understand the effect of inserting and removing just setups in our current schedule.

The first of these neighborhoods is the Setup Insertion. It works by swiping the machines searching for two consecutive operations of the same family. Once a pair of subsequent operations of the same family is found, we insert a setup of the corresponding family between them. An illustrative example is show in Figure 4.19. 


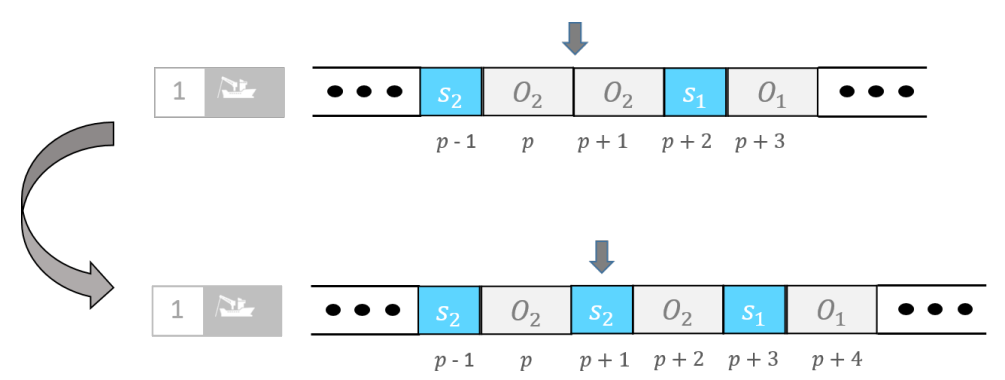

Figure 4.19: Setup Insertion

Source: The Author

The second neighborhood is the Setup Removal. It works by searching in the machines a sequence of: operation - setup time - operation, all from the same family. Then it removes this intermediate setup, transforming two batches in a single one. An illustrative example is exposed in Figure 4.20.

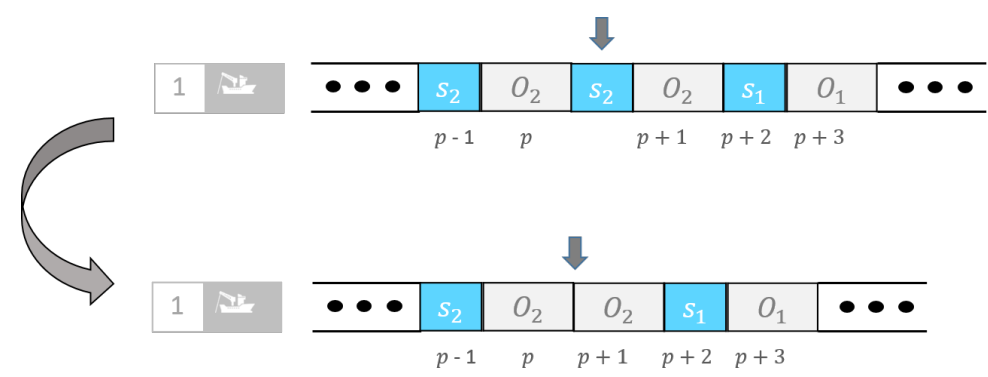

Figure 4.20: Setup Removal

Source: The Author

These neighborhoods are especially important when combined to the infeasibility strategy that will be explained in the next subsection. The Setup Removal creates batches of multiple operations, that may disrespect the occupation constraints, helping to reach infeasible solution space. The Setup Insertion is crucial to return to the feasible solution space, by dividing long batches that disrespect occupation constraints into feasible batches. It is important to notice that these neighborhoods must be combined with the Swap and Relocate neighborhoods, in order to avoid returning to the same initial solution, since they have the exact opposite effect in the current schedule.

\subsection{6}

\section{Infeasibility Strategy}

One important consideration on the proposed neighborhood structures is that we relax some constraints and keep some constraints hard. Basically, we relax the occupation constraints, allowing batches to have operations that exceed 
the machine occupation limit. The reason to allow this violation on constraints is to enlarge the solution space, going to infeasible space and returning to feasible solutions that we could not reach if we did not pass by infeasible solutions before. The family and eligibility constraints are kept hard because the movements to reach feasible solutions would only go back to previously evaluated solutions. The infeasible solutions are penalized by the formula:

$$
p(s)=\alpha c(s)
$$

The penalized solution is denoted by $p(s)$, the current solution, $c(s)$, and $\alpha$ is the penalty factor. The initial value of $\alpha$ is 100 and was obtained by testing values from 10 to 200, with steps of size 10 . The main idea is to increase the penalty for infeasible solutions that are reached, avoiding the search to go far from the feasible space. For that, $\alpha$ is enlarged in $10 \%$ at each step where an infeasible solution is considered and reduced in $5 \%$ when a feasible solution is reached. That way we can manipulate $\alpha$ to be in a reasonable value and not diverge from the feasible solutions space.

\section{4 \\ Local Search}

After defining the neighborhood structures and infeasibility strategy we propose a local search that is used to recurrently find improved solutions for the problem. There are two main characteristics of this Local Search, which are described in the subsections that follow: the First Improvement rule and the Random Variable Neighborhood Descent (RVND).

\subsection{1}

\section{First Improvement}

The proposed Local Search follows the First Improvement rule. This means that the algorithm stops when it finds the first improved solution when searching in a specific neighborhood. The other possible strategy is to implement a Best Improvement rule, which evaluates all possible neighbors solutions and then selects the best of them. We chose the First Improvement rule in order to save time and computational effort while performing the Local Search. 


\subsection{2 \\ Random Variable Neighborhood Descent}

The other important characteristic of our local search is the Random Variable Neighborhood Descent (RVND). While performing the local search we list all the neighborhood structures detailed in the previous subsections and randomly select one of them. If the solution provided is better than the current solution, we update the current solution and shuffle again the list with neighborhood structures, including all four neighborhoods. If the solution provided is worse than the current solution we remove the used neighborhood structure from the list and randomly select the next. This algorithm goes on until all neighborhood structures have been removed from the list or when a improved solution is achieved. In Algorithm 2 the pseudocode for the RVND is described.

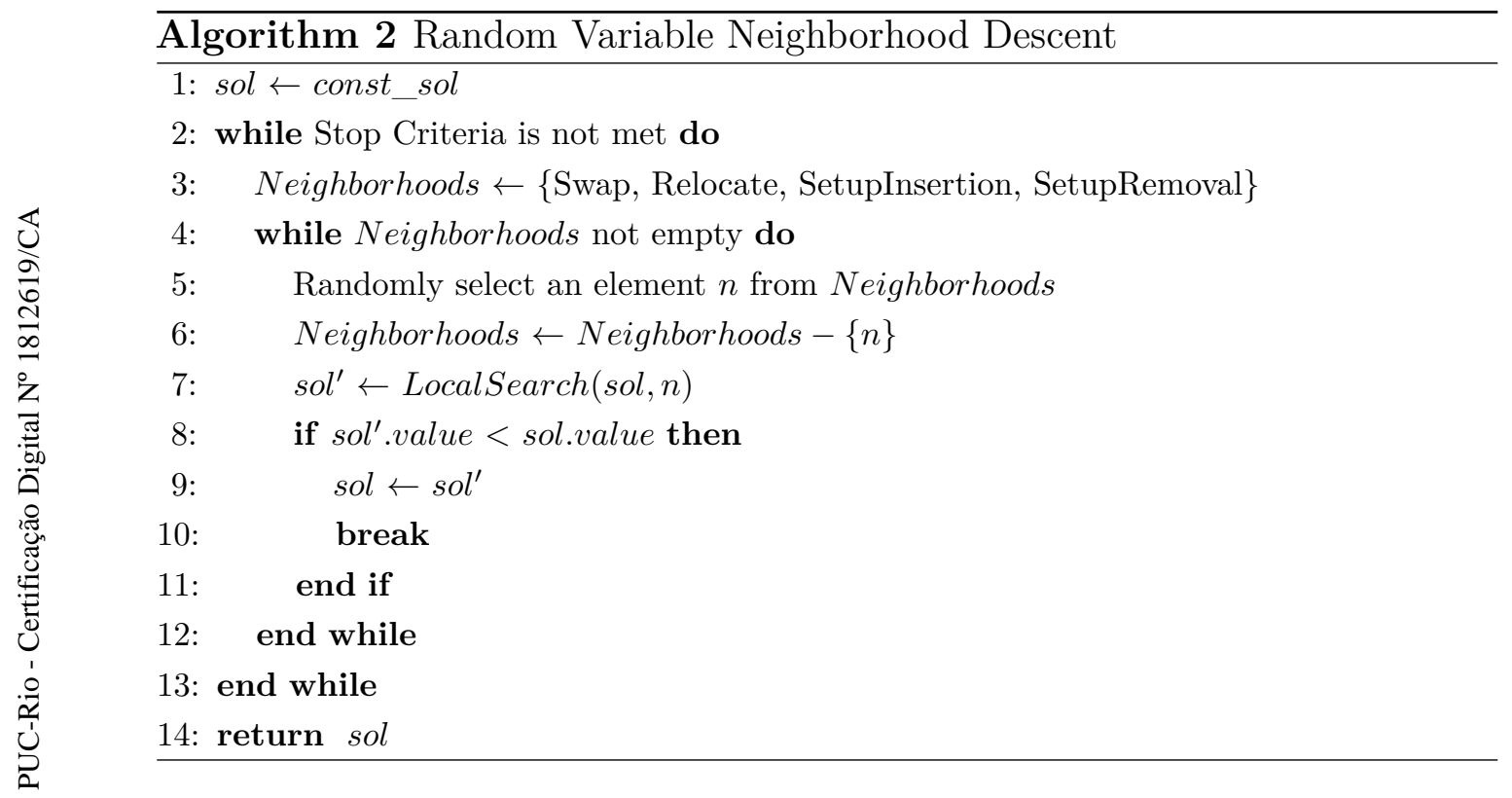

\section{5}

\section{Iterated Local Search}

After we described the neighborhood structures and the local search characteristics, we are able to introduce the proposed Iterated Local Search for the identical parallel machine scheduling problem. This metaheuristic consists on first running the Schedule Construction Procedure method explained in subsection 4.2.1 and storing the five best different solutions, in decreasing order. These solutions will be used as input in a multi-start strategy. In each multi-start loop, we define the Schedule Construction Procedure solution and set it as our initial solution. Then the method starts the Iterated Local Search procedure by setting it as our best and current solution, and run the local 
search. After it, we use a random function to select two machines and positions to perturb the current solution. Then we run the Local Search procedures again and evaluate: if a better solution is found and it is feasible, it is stored as the best solution and the current solution is updated; if we find a better solution but it is infeasible then only the current solution is updated; if the reached solution is worse than our best solution we use a simulated annealing acceptance criteria that will be explained in section 4.5.2 to define if we update our current solution or not. Another characteristic of the Iterated Local Search is the starting point strategy. If we have $\lambda$ iterations without improving our best solution, we set the current solution as our best solution, avoiding solutions to diverge from good solutions that already have been reached.

After that, the next round of the multi-start procedure is performed, by setting the next best solution from the Constructive Decision as the current solution and repeating the whole Iterated Local Search algorithm. This multistart is done with the five best solutions reached in the Constructive Decision, and the best solution among all solutions achieved in the whole method is stored as the final result. The pseudocode of the Iterated Local Search is presented in Algorithm 3.

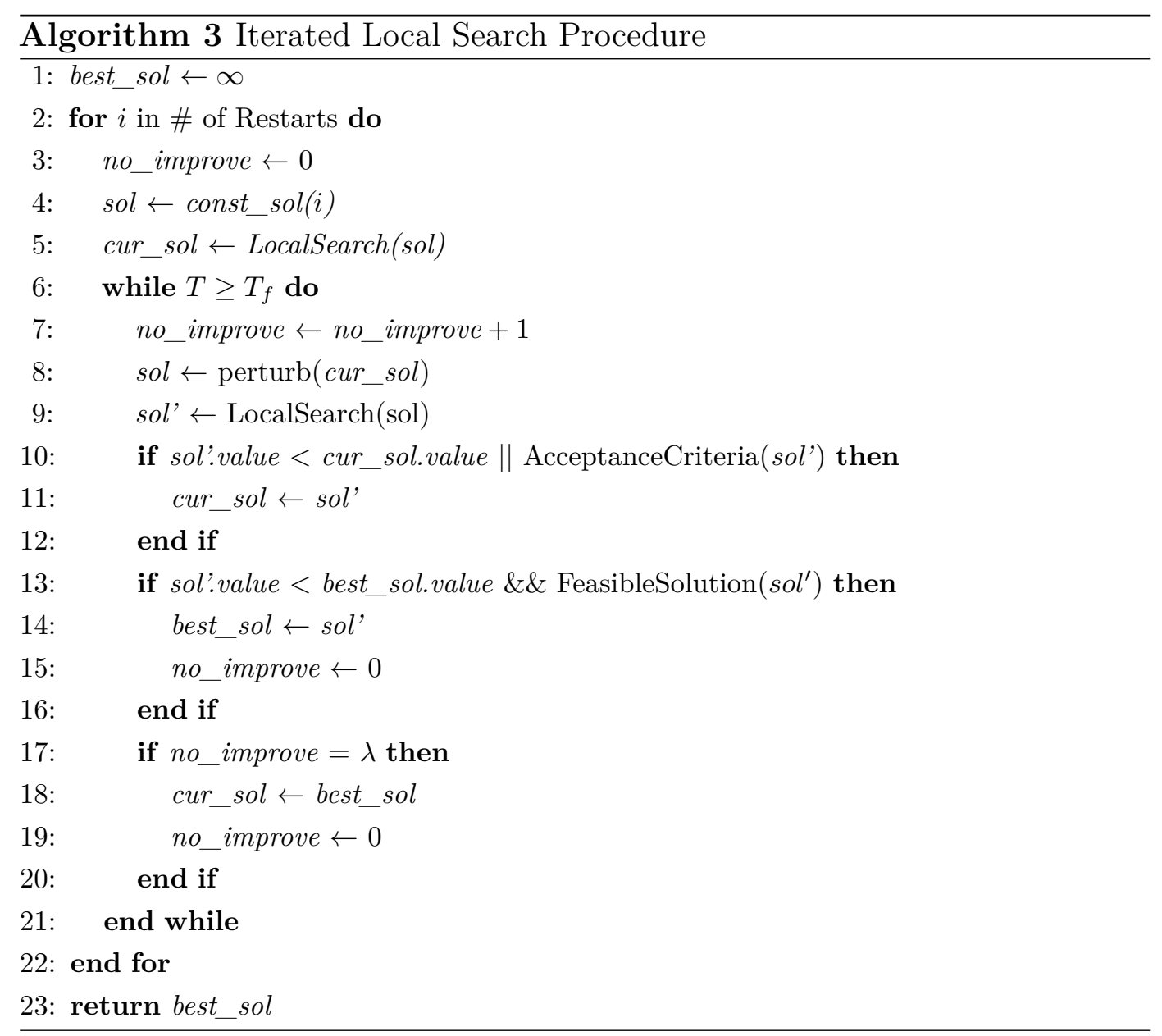




\subsection{1 \\ Perturbation}

We defined the number of elements that we will perturb in our current solution, in each iteration, and how this perturbation will occur. To perturb the solutions we randomly choose two positions and two machines and one of the neighborhoods: Swap or Relocate. If the Swap structure is chosen, we change these operations position. If the Relocate structure is chosen, we relocate the operation from the first machine and position to the second.

\subsection{2}

\section{Acceptance Criteria}

In this work we used the simulated annealing acceptance criteria, proposed by Kirkpatrick et al. (1983) to decide whether or not we accept worse solutions in our iterated local search. This criteria is based on defining a probability to decide if we accept a worse solution at each iteration. This probability is defined by the formula:

$$
p(T)=\exp \left(\frac{-d i f}{T}\right)
$$

dif is defined as sol - cur_sol so worse solutions that are closer to the current solution have better chances to be accepted. $T$ is the current temperature, that is updated at each iteration, being multiplied by the decreasing factor $\delta$. The initial temperature $T_{i}$ usually starts with a large value, in order to diversify the solution acceptance in the beginning of the method and at each iteration it is decreased to have less chance of accepting poorer solutions. Based on Pisinger and Røpke (2007), we defined the initial temperature $T_{i}$ and final temperature $T_{f}$. The initial temperature $T_{i}$ is set to accept with 0.5 probability solutions that are $40 \%$ worse than the current solution provided by the constructive heuristic following the formula in equation $4-1$.

$$
T_{i}=-\frac{0.4 \times \text { cur_sol }}{\ln (0.5)}
$$

We use the final temperature $T_{f}$ to define the end of the iterated local search, so we stop it when $T$ is the temperature that accept with 0.5 probability solutions that are $0.01 \%$ worse than the current solution, following the formula in equation $4-2$.

$$
T_{f}=-\frac{0.0001 \times \text { cur_sol }}{\ln (0.5)}
$$


As we want our iterated local search to run $\eta$ iterations, we define the decreasing factor $\delta$ to decrease the initial temperature $T_{i}$ to reach the stop temperature value $T_{f}$ in $\eta$ iterations, following the formula in equation 4-3.

$$
\delta=\left(\frac{T_{f}}{T_{i}}\right)^{\left(\frac{1}{\eta}\right)}
$$




\section{5}

\section{Computational Experiments}

In this section, we describe all computational experiments performed and provide analysis. We tested our Iterated Local Search metaheuristic with the whole method described in Section 4.5 and defined it as the ILS - Complete (ils). We also ran tests for seven variations, changing the neighborhoods by enabling just the swap neighborhood, which we call ILS OnlySwap (ils-os); enabling just the relocate neighborhood, which we call ILS - OnlyRelocate (ils-or); disabling only the swap neighborhood, the ILS - NoSwap (ils-ns); disabling only the relocate neighborhood, the ILS NoRelocate (ils-nr); disabling only the setup insertion neighborhood, the ILS

- NoSetupInsertion (ils-nsi); disabling only the setup removal neighborhood, the ILS - NoSetupRemoval (ils-nsr); and the whole ILS method but disabling the infeasibility strategy, the ILS - NoInfeasibility (ils-ni).

Since we have random methods on our perturbation procedures, simulated annealing acceptance criteria and variable neighborhood descent, we defined a set of ten different seeds that are used as inputs in our tests, running ten times each method in each instance. We ran the experiments on a computer with $64 \mathrm{~GB}$ of RAM and Intel Core i7-8700K CPU of $3.70 \mathrm{GHz}$, using $\mathrm{C}++$ for developing the metaheuristic and running Linux.

\section{1}

\section{Instances Description}

All tests were performed on a set of 72 PLSV instances, proposed by Abu-Marrul et al. (2020a), and available in Abu-Marrul et al. (2019), with $|\mathcal{M}|=\{4,8\}$, and $|\mathcal{O}|=\{15,25,50\}$. Each combination of the number of Machines and Operations defines a group. Group 1 is defined as $|\mathcal{O}|=\{15\}$, $|\mathcal{M}|=\{4\}$. Group 2 as $|\mathcal{O}|=\{15\},|\mathcal{M}|=\{8\}$. Group 3 as $|\mathcal{O}|=\{25\}$, $|\mathcal{M}|=\{4\}$. Group 4 as $|\mathcal{O}|=\{25\},|\mathcal{M}|=\{8\}$. Group 5 as $|\mathcal{O}|=\{50\}$, $|\mathcal{M}|=\{4\}$. Group 6 as $|\mathcal{O}|=\{50\},|\mathcal{M}|=\{8\}$.

Each group contains 12 instances, formed by the combination of 3 input factors in the instance generation: the release date factor $\alpha=\{0.25,0.50,0.75\}$, which for lower values sets the release dates closer to the beginning of the 
time horizon; the eligibility factor $\beta=\{0.7,0.9\}$, which for values closer to one generate more number of eligible operations to a machine; and the association factor $\gamma=\{0.05,0.15\}$, defining the probability of associating a specific job to an operation.

\section{2}

\section{Parameters}

The Iterated Local Search algorithm has three important parameters that are tuned by testing a set of values to these parameters. The first of them is the number of iterations $\eta$. We tested the method running from 100 to 1000 iterations, with steps of size 100 and decided to stop the algorithm after 500 iterations, since the results rarely improved after it. Another parameter tuned is the number of iterations without improvement, $\lambda$, to define the starting point strategy. We tested $\lambda$ from $5 \%$ to $50 \%$ of the number of iterations, with steps of size $5 \%$ and the best outcomes for the method were achieved with $\lambda$ $=10 \%$. The third parameter is the number of elements that we perturb in each iteration. It is defined as $15 \%$ of the number of operations of the current instance, rounding up this value if it is not an integer. This value was tuned after a sensitivity analysis, testing all possible percentages going from $5 \%$ to $50 \%$ with steps of size $5 \%$. Perturbing a small number of operations did not diversify the solution space and a large number of operations set the current solution too far from the best solution achieved.

\section{3 \\ Results}

In this section, we present the final results of our tests. As mentioned earlier we compare our complete method with variations that disable some neighborhoods and the infeasibility strategy. The main criteria that we compare the methods are the computational time and relative percentage deviation, computed as $R P D=\left(\frac{T W C-B K S}{B K S}\right) \times 100$. Where $T W C$ is the total weighted completion time obtained by the method in the specific instance and the $B K S$ is the best known solution. The $B K S$ for our instances was obtained by the mathematical formulations proposed by Abu-Marrul et al. (2020a), with computational time of six hours.

Since we run ten times each instance for each method, we gathered the minimum $\left(R P D^{-}\right)$, maximum $\left(R P D^{+}\right)$and average $(\overline{R P D}) R P D$. We present the $R P D$ standard deviation $\left(\sigma_{R P D}\right)$, indicating the methods that have more stable behaviour. The $R P D_{L B}^{-}$computed as $R P D_{L B}^{-}=\left(\frac{L B-B K S}{B K S}\right) \times 100$, where 
$L B$ is the Lower Bound of the instance, indicating how far from the Lower Bound the method is in an specific instance. We also defined the number of improvements (\#Imp) for each method, that consists on, for each method and group, counting the number of solutions reached that are equal or better than the $B K S$. As each group has twelve instances that we ran ten times each, the maximum number of improvements for each method in each group is 120 . The $\%$ Imp is obtained by dividing \#Imp by 120 . Another relevant consideration that is not described in the results tables is that the four methods, ILS - Complete, ILS - NoInfeasibility, ILS - NoSetupInsertion and ILS NoSetupRemoval were able to find or improve the previous $B K S$, at least once, in all 72 instances. The complete results for each instance is presented in this thesis appendix A.

Table 5.1: Results for Group 1

\begin{tabular}{rlrrrrrrrr}
\hline Instance & Method & $R P D^{-}$ & $\overline{R P D}$ & $R P D^{+}$ & $\sigma_{R P D}$ & $R P D_{L B}^{-}$ & \#Imp & \% Imp & $\overline{\text { Time }}$ \\
\hline \multirow{5}{*}{} & ils & $\mathbf{0 . 0 0 0}$ & 0.005 & $\mathbf{0 . 1 2 1}$ & 0.024 & $\mathbf{0}$ & 115 & $95.8 \%$ & 0.658 \\
& ils-ni & $\mathbf{0 . 0 0 0}$ & 0.003 & $\mathbf{0 . 1 2 1}$ & 0.019 & $\mathbf{0}$ & 117 & $97.5 \%$ & 0.667 \\
$|\mathcal{O}|=\{15\}$ & ils-nr & $\mathbf{0 . 0 0 0}$ & 0.363 & 5.658 & 0.786 & $\mathbf{0}$ & 55 & $45.8 \%$ & 0.270 \\
$|\mathcal{M}|=\{4\}$ & ils-ns & $\mathbf{0 . 0 0 0}$ & 3.602 & 6.236 & 2.198 & $\mathbf{0}$ & 10 & $8.3 \%$ & 0.349 \\
& ils-nsi & $\mathbf{0 . 0 0 0}$ & 0.003 & $\mathbf{0 . 1 2 1}$ & 0.019 & $\mathbf{0}$ & 117 & $97.5 \%$ & 0.686 \\
& ils-nsr & $\mathbf{0 . 0 0 0}$ & $\mathbf{0 . 0 0 1}$ & $\mathbf{0 . 1 2 1}$ & $\mathbf{0 . 0 1 1}$ & $\mathbf{0}$ & $\mathbf{1 1 9}$ & $\mathbf{9 9 . 2 \%}$ & 0.690 \\
& ils-or & $\mathbf{0 . 0 0 0}$ & 3.570 & 6.236 & 2.175 & $\mathbf{0}$ & 10 & $8.3 \%$ & 0.352 \\
& ils-os & $\mathbf{0 . 0 0 0}$ & 0.926 & 5.960 & 1.364 & $\mathbf{0}$ & 40 & $33.3 \%$ & $\mathbf{0 . 2 2 6}$ \\
\hline
\end{tabular}

In Table 5.1, we see how the different methods performance in the first group of instances. Since Group 1 is formed by small instances, the $B K S$ in that group are the proved optimal solutions. We can see that the ILS - Complete, ILS - NoInfeasibility, ILS - NoSetupInsertion and ILS NoSetupRemoval have good performances in this group of instances, reaching the optimal solution in more than $95 \%$ of the tests and have very similar computational time. The ILS - NoSetupRemoval has the best performance among them all, reaching the optimal solution in $99 \%$ of the tests and having the best average $R P D$ and $R P D$ standard deviation.

Table 5.2: Results for Group 2

\begin{tabular}{lllrrrrrrr}
\hline Instance & Method & $R P D^{-}$ & $\overline{R P D}$ & $R P D^{+}$ & $\sigma_{R P D}$ & $R P D_{L B}^{-}$ & \#Imp & \% Imp & $\overline{\text { Time }}$ \\
\hline \multirow{5}{*}{} & ils & $\mathbf{0 . 0 0 0}$ & 0.117 & 3.180 & 0.463 & $\mathbf{0}$ & 108 & $90.0 \%$ & 0.741 \\
& ils-ni & $\mathbf{0 . 0 0 0}$ & $\mathbf{0 . 0 6 1}$ & $\mathbf{1 . 1 9 6}$ & $\mathbf{0 . 1 9 7}$ & $\mathbf{0}$ & 105 & $87.5 \%$ & 0.745 \\
$|\mathcal{O}|=\{15\}$ & ils-nr & $\mathbf{0 . 0 0 0}$ & 0.928 & 5.783 & 1.175 & $\mathbf{0}$ & 22 & $18.3 \%$ & 0.298 \\
$|\mathcal{M}|=\{8\}$ & ils-ns & $\mathbf{0 . 0 0 0}$ & 2.561 & 8.234 & 2.423 & $\mathbf{0}$ & 10 & $8.3 \%$ & 0.387 \\
& ils-nsi & $\mathbf{0 . 0 0 0}$ & 0.090 & 2.174 & 0.302 & $\mathbf{0}$ & 103 & $85.8 \%$ & 0.746 \\
& ils-nsr & $\mathbf{0 . 0 0 0}$ & 0.065 & 2.174 & 0.267 & $\mathbf{0}$ & $\mathbf{1 1 0}$ & $\mathbf{9 1 . 7 \%}$ & 0.747 \\
& ils-or & $\mathbf{0 . 0 0 0}$ & 2.854 & 8.234 & 2.417 & $\mathbf{0}$ & 10 & $8.3 \%$ & 0.381 \\
& ils-os & $\mathbf{0 . 0 0 0}$ & 1.038 & 4.722 & 1.185 & $\mathbf{0}$ & 24 & $20.0 \%$ & $\mathbf{0 . 2 5 2}$ \\
\hline
\end{tabular}

In Table 5.2 we present the results related to the second group of instances, which the $B K S$ solutions are again the proved optimal solutions. In this group, 
the same four methods have good performance, reaching the optimal solutions in more than $85 \%$ of the tests. In this group, it is not clear which is the best method. Again the ILS - NoSetupRemoval reaches more optimal solutions than the other methods, but if we look the $R P D$ average and, especially, the standard deviation indicating that the method is more stable and reaches less often poorer solutions, the ILS - NoInfeasibility is the best method, reaching, in average, better solutions and also in the worst case scenario.

Table 5.3: Results for Group 3

\begin{tabular}{llrrrrrrrr}
\hline Instance & Method & \multicolumn{1}{c}{$R P D^{-}$} & $\overline{R P D}$ & $R P D^{+}$ & $\sigma_{R P D}$ & $R P D_{L B}^{-}$ & \#Imp & \%Imp & $\overline{\text { Time }}$ \\
\hline \multirow{6}{*}{} & ils & $\mathbf{- 2 . 0 8 4}$ & -0.509 & $\mathbf{0 . 1 6 5}$ & 0.664 & $\mathbf{0}$ & $\mathbf{1 0 9}$ & $\mathbf{9 0 . 8 \%}$ & 2.902 \\
& ils-ni & $\mathbf{- 2 . 0 8 4}$ & -0.508 & $\mathbf{0 . 1 6 5}$ & $\mathbf{0 . 6 5 8}$ & $\mathbf{0}$ & $\mathbf{1 0 9}$ & $\mathbf{9 0 . 8 \%}$ & 2.839 \\
$|\mathcal{O}|=\{25\}$ & ils-nr & $\mathbf{- 2 . 0 8 4}$ & 0.240 & 2.883 & 0.920 & 0.704 & 33 & $27.5 \%$ & 1.028 \\
& ils-ns & 0.110 & 3.210 & 5.277 & 1.511 & 1.085 & 0 & $0.0 \%$ & 1.119 \\
$|\mathcal{M}|=\{4\}$ & ils-nsi & $\mathbf{- 2 . 0 8 4}$ & -0.507 & 0.269 & 0.660 & $\mathbf{0}$ & 108 & $90.0 \%$ & 3.041 \\
& ils-nsr & $\mathbf{- 2 . 0 8 4}$ & $\mathbf{- 0 . 5 2 3}$ & 0.269 & 0.667 & $\mathbf{0}$ & 107 & $89.2 \%$ & 2.945 \\
& ils-or & 0.110 & 2.845 & 5.159 & 1.530 & 1.085 & 0 & $0.0 \%$ & 1.163 \\
& ils-os & -1.679 & 0.405 & 2.555 & 0.918 & 0.704 & 27 & $22.5 \%$ & $\mathbf{0 . 9 7 0}$ \\
\hline
\end{tabular}

In Table 5.3 we present the results among the third group of instances. From this group forward, since the instances are larger, the $B K S$ target solutions are not optimal, so we are able to find better solutions than the previous $B K S$ and consequently negative $R P D$ s. The computational times for that group are slightly higher. The same four methods have the best performances, reaching $B K S$ or better solutions in about $90 \%$ of the tests and are almost tied in all RPD metrics, with a very small advantage to the ILS NoInfeasibility in the standard deviation and to the ILS - NoSetupRemoval in the average. Regarding the worst case scenario the ILS - Complete and ILS - NoInfeasibility are the best.

Table 5.4: Results for Group 4

\begin{tabular}{llrrrrrrrr}
\hline Instance & Method & \multicolumn{1}{c}{$R P D^{-}$} & $\overline{R P D}$ & $R P D^{+}$ & $\sigma_{R P D}$ & $R P D_{L B}^{-}$ & $\#$ Imp & \%Imp & $\overline{T i m e}$ \\
\hline & ils & $\mathbf{- 2 . 3 6 2}$ & -0.473 & 0.946 & 0.693 & $\mathbf{0 . 0 0 2}$ & 104 & $86.7 \%$ & 3.381 \\
& ils-ni & $\mathbf{- 2 . 3 6 2}$ & -0.409 & $\mathbf{0 . 6 7 8}$ & 0.668 & $\mathbf{0 . 0 0 2}$ & 98 & $81.7 \%$ & 3.275 \\
$|\mathcal{O}|=\{25\}$ & ils-nr & -1.938 & 0.671 & 3.077 & 1.112 & 0.064 & 23 & $19.2 \%$ & 1.153 \\
$|\mathcal{M}|=\{8\}$ & ils-ns & 2.151 & 4.197 & 6.520 & 1.390 & 3.314 & 0 & $0.0 \%$ & 1.300 \\
& ils-nsi & $\mathbf{- 2 . 3 6 2}$ & $\mathbf{- 0 . 4 7 7}$ & 0.783 & 0.709 & $\mathbf{0 . 0 0 2}$ & 100 & $83.3 \%$ & 3.490 \\
& ils-nsr & $\mathbf{- 2 . 3 6 2}$ & -0.457 & 0.802 & $\mathbf{0 . 6 6 3}$ & $\mathbf{0 . 0 0 2}$ & $\mathbf{1 0 9}$ & $\mathbf{9 0 . 8 \%}$ & 3.401 \\
& ils-or & 2.054 & 4.523 & 6.811 & 1.302 & 3.611 & 0 & $0.0 \%$ & 1.300 \\
& ils-os & -1.938 & 0.884 & 3.505 & 1.107 & 0.064 & 16 & $13.3 \%$ & $\mathbf{1 . 0 7 2}$ \\
\hline
\end{tabular}

In Table 5.4, the forth group of instances is exposed. Again the four methods have good performances, reaching or beating the target method in more than $80 \%$ of the tests. In terms of RPD average, the ILS - NoSetupInsertion has the best performance, almost tied with the ILS - Complete. In terms of standard deviation, the ILS - NoInfeasibility and the ILS - NoSetupRemoval 
are the best, having a more predictable behavior. In terms of worst case scenario, the ILS - NoInfeasibility leads.

Table 5.5: Results for Group 5

\begin{tabular}{llrrrrrrrr}
\hline Instance & Method & \multicolumn{1}{c}{$R P D^{-}$} & $\overline{R P D}$ & $R P D^{+}$ & $\sigma_{R P D}$ & $R P D_{L B}^{-}$ & \#Imp & \%Imp & $\overline{\text { Time }}$ \\
\hline \multirow{6}{*}{} & ils & -7.918 & -3.539 & -0.650 & 1.948 & 3.553 & $\mathbf{1 2 0}$ & $\mathbf{1 0 0 . 0 \%}$ & 38.929 \\
& ils-ni & -7.703 & -3.431 & -0.661 & 1.883 & $\mathbf{3 . 4 0 8}$ & $\mathbf{1 2 0}$ & $\mathbf{1 0 0 . 0 \%}$ & 30.582 \\
$|\mathcal{O}|=\{50\}$ & ils-nr & -6.895 & -2.426 & 0.717 & $\mathbf{1 . 8 5 0}$ & 3.960 & 112 & $93.3 \%$ & 11.853 \\
$|\mathcal{M}|=\{4\}$ & ils-ns & -2.976 & 1.047 & 4.561 & 2.213 & 8.954 & 40 & $33.3 \%$ & $\mathbf{7 . 2 8 4}$ \\
& ils-nsi & $\mathbf{- 7 . 9 3 4}$ & -3.500 & $\mathbf{- 0 . 6 6 3}$ & 1.893 & 3.575 & $\mathbf{1 2 0}$ & $\mathbf{1 0 0 . 0 \%}$ & 40.645 \\
& ils-nsr & -7.910 & $\mathbf{- 3 . 5 4 4}$ & -0.602 & 1.940 & 3.508 & $\mathbf{1 2 0}$ & $\mathbf{1 0 0 . 0 \%}$ & 40.808 \\
& ils-or & -2.777 & 1.039 & 4.303 & 1.992 & 8.751 & 30 & $25.0 \%$ & 7.719 \\
& ils-os & -7.004 & -2.262 & 0.711 & 1.857 & 4.422 & 107 & $89.2 \%$ & 12.920 \\
\hline
\end{tabular}

In Table 5.5, the fifth group of instances is presented. In groups 5 and 6 , we have 50 operations per instance, leading to considerably larger computational times. In this group, besides the four methods with the presumably good performances, the ILS - NoRelocate and ILS - OnlySwap also find equal or better solutions than the $B K S$ in about $90 \%$ of the tests. In larger instances, the Iterated Local Search recurrently finds improved solutions, and the four leading methods of the other groups find the previous $B K S$ solutions or better in all tests. In average, the ILS - Complete has the better results but the variance is larger, while the ILS - NoInfeasibility has a more stable behavior. In terms of best case scenario, the ILS - NoSetupInsertion has the best performance and in the worst case scenario the ILS - NoSetupRemoval is the best.

Table 5.6: Results for Group 6

\begin{tabular}{rlrrrrrrrr}
\hline Instance & Method & \multicolumn{1}{c}{$R P D^{-}$} & $\overline{R P D}$ & $R P D^{+}$ & $\sigma_{R P D}$ & $R P D_{L B}^{-}$ & \#Imp & \%Imp & $\overline{\text { Time }}$ \\
\hline \multirow{2}{*}{} & ils & -11.021 & $\mathbf{- 5 . 1 9 4}$ & $\mathbf{- 1 . 5 8 6}$ & 2.638 & $\mathbf{3 . 0 8 1}$ & $\mathbf{1 2 0}$ & $\mathbf{1 0 0 . 0 \%}$ & 40.286 \\
& ils-ni & -11.112 & -5.039 & -1.424 & 2.638 & 3.438 & $\mathbf{1 2 0}$ & $\mathbf{1 0 0 . 0 \%}$ & 31.035 \\
$|\mathcal{O}|=\{50\}$ & ils-nr & -10.287 & -3.865 & 1.947 & 2.952 & 4.134 & 110 & $91.7 \%$ & 11.702 \\
$|\mathcal{M}|=\{8\}$ & ils-ns & -5.829 & -0.904 & 2.348 & 2.342 & 8.538 & 80 & $66.7 \%$ & $\mathbf{7 . 5 8 6}$ \\
& ils-nsi & -10.809 & -5.162 & -1.365 & 2.659 & 3.693 & $\mathbf{1 2 0}$ & $\mathbf{1 0 0 . 0 \%}$ & 41.913 \\
& ils-nsr & $\mathbf{- 1 1 . 4 2 9}$ & -5.174 & -1.554 & 2.691 & 3.318 & $\mathbf{1 2 0}$ & $\mathbf{1 0 0 . 0 \%}$ & 41.677 \\
& ils-or & -5.101 & -0.936 & 2.048 & $\mathbf{2 . 3 0 2}$ & 7.155 & 80 & $66.7 \%$ & 7.922 \\
& ils-os & -9.857 & -3.656 & 2.044 & 2.919 & 3.979 & 110 & $91.7 \%$ & 12.361 \\
\hline
\end{tabular}

In Table 5.6, we observe that the results of Group 6 are similar to the previous on Group 5. The computational time are slightly higher since we have more machines. In terms of average, the ILS - Complete has the best performance and in terms of standard deviation, it is almost tied with the ILS - NoInfeasibilty. In terms of best case scenario, the ILS - NoSetupRemoval reached the best solution and in terms of worst case scenario, the ILS NoSetupInsertion has the best result.

Another important way to analyse the described methods and solutions achieved is by using boxplots of the $R P D$ for each method. The graphic is 
exposed in Figure 5.1, where all methods are described and the database contains all groups of instances. Analysing the graphic, we can see that the ILS - NoSwap and the ILS - OnlyRelocate are the worst methods, finding positive $R P D$ s in average. This analysis indicates how important the swap neighborhood is to the metaheuristic, where the methods that do not enable this neighborhood have notably the worst performances.

The ILS - NoRelocate and the ILS - OnlySwap are considerably better than the methods that do not consider the swap neighborhood, but still have positive or null $R P D$ averages. Indicating that not only the swap neighborhood is crucial to the metaheuristic but also the combination of swap and relocate neighborhoods.

As we saw in the first results tables, the methods ILS - Complete, ILS - NoInfeasibility, ILS - NoSetupInsertion and ILS - NoSetupRemoval have similar performances. In terms of RPD median the ILS NoSetupRemoval has a slight advantage among them all, while the ILS - Complete has the lower fence. In terms of worst case scenario the ILS NoInfeasibility leads as the best method.

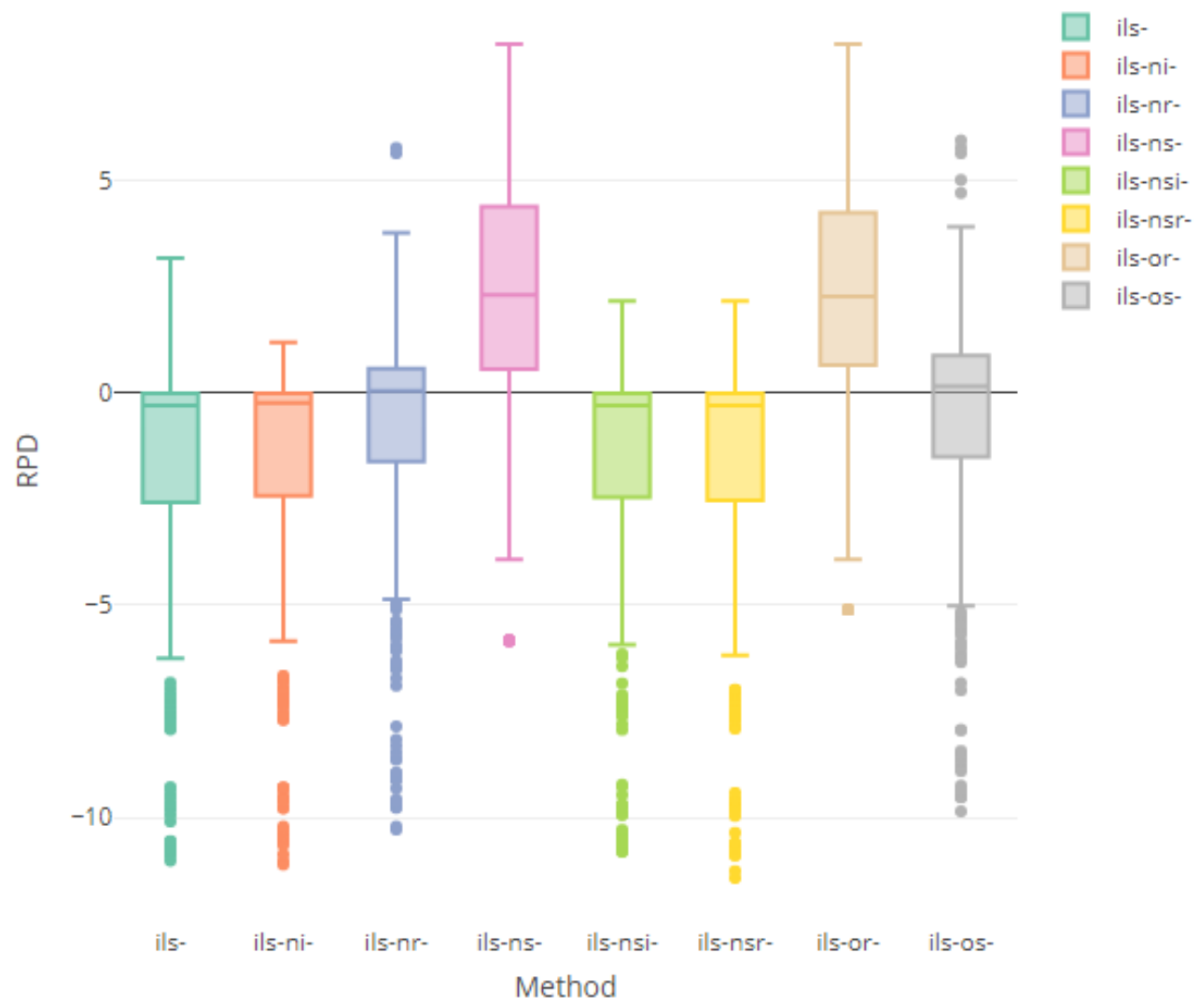

Figure 5.1: Boxplot - All Groups 
Another way to compare our methods is to compare the average $R P D$ and average computational time. If one method is faster than the other and also have lower average $R P D$, we can affirm that this method is dominant. In Figure 5.2, we can see that the method ILS - NoSwap is dominant when compared to the ILS - OnlyRelocate, the ILS - NoRelocate is dominant regarding the ILS - OnlySwap and the ILS - NoSetupRemoval dominates the ILS - NoSetupInsertion. With that analysis it is notable that the dominated methods can be discarded, since there are other faster methods that reach better outcomes.

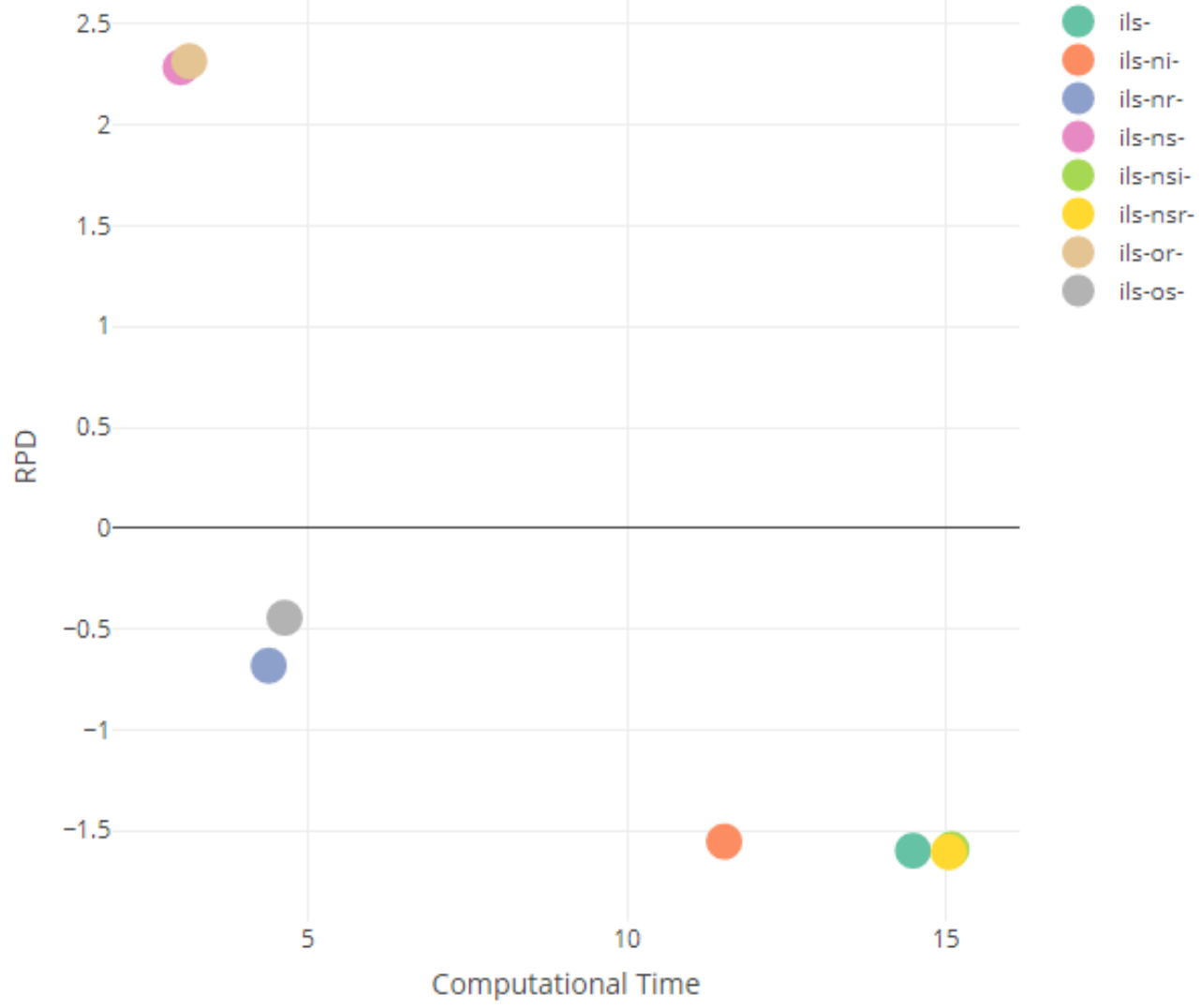

Figure 5.2: Dominance Among Methods

\section{4}

\section{Statistical Tests}

After analysing the results and graphics, we notice an advantage for four of the proposed methods, they are: ILS - Complete, ILS - NoInfeasibility, ILS - NoSetupInsertion and ILS - NoSetupRemoval. To define if we have significant statistical differences between the methods, we ran the ANOVA statistical test, using the software RStudio, within all pairs of methods. We 
defined a significance level or $5 \%$, so to reject the null hypothesis that the two methods do not have statistical significant differences the $p$-value obtained must be equal or lower than 0.05 . The results of the statistical tests are shown in Table 5.7.

Table 5.7: ANOVA Tests Within All Pairs of Methods

\begin{tabular}{lcc}
\hline Pair of Methods & $p \_$value & Statistical Difference \\
\hline ils-nr/ils & 0.000 & Yes \\
ils-ns/ils & 0.000 & Yes \\
ils-or/ils & 0.000 & Yes \\
ils-os/ils & 0.000 & Yes \\
ils-nr/ils-ni & 0.000 & Yes \\
ils-ns/ils-ni & 0.000 & Yes \\
ils-or/ils-ni & 0.000 & Yes \\
ils-os/ils-ni & 0.000 & Yes \\
ils-ns/ils-nr & 0.000 & Yes \\
ils-nsi/ils-nr & 0.000 & Yes \\
ils-nsr/ils-nr & 0.000 & Yes \\
ils-or/ils-nr & 0.000 & Yes \\
ils-nsi/ils-ns & 0.000 & Yes \\
ils-nsr/ils-ns & 0.000 & Yes \\
ils-os/ils-ns & 0.000 & Yes \\
ils-or/ils-nsi & 0.000 & Yes \\
ils-os/ils-nsi & 0.000 & Yes \\
ils-or/ils-nsr & 0.000 & Yes \\
ils-os/ils-nsr & 0.000 & Yes \\
ils-os/ils-or & 0.000 & Yes \\
ils-ni/ils & 1.000 & No \\
ils-nsi/ils & 1.000 & No \\
ils-nsr/ils & 1.000 & No \\
ils-nsi/ils-ni & 1.000 & No \\
ils-nsr/ils-ni & 1.000 & No \\
ils-os/ils-nr & 0.624 & No \\
ils-or/ils-ns & 1.000 & No \\
ils-nsr/ils-nsi & 1.000 & \\
\hline & &
\end{tabular}

As we can expect based on the previous results and graphics, there are significant statistical difference between all pairs formed by one of the four worst methods (ILS - NoSwap, ILS - OnlySwap, ILS - NoRelocate and ILS - OnlyRelocate) and one of the four best methods (ILS - Complete, ILS NoInfeasibility, ILS - NoSetupInsertion and ILS - NoSetupRemoval), which we could observe in the boxplots graphics, where the outcomes of the four best methods are considerably better than the four worst methods results.

Among the pairs formed by two of the four best methods, there are no 
significant statistical difference between them when analysing all groups of instances. It is possible that in larger, more complex instances the differences between these methods become more notable.

There is also no significant difference between the pair of methods ILS - OnlySwap/ILS - NoRelocate and ILS - OnlyRelocate/ILS - NoSwap. It is reasonable since these pairs of methods have the same main neighborhood structures of our problem: the Swap or Relocate, but not both.

Among the four worst methods we can see that the ones that consider the Swap neighborhood are notably better than the ones that consider the Relocate neighborhood. We can observe that there are statistical differences between these pairs of methods (ILS - NoSwap/ILS - NoRelocate, ILS OnlyRelocate/ ILS - NoRelocate, ILS - OnlySwap/ ILS - NoSwap and ILS - OnlySwap/ILS - OnlyRelocate). 


\section{6}

\section{Conclusions and Future Work}

The objective of this work of proposing a metaheuristic to solve identical parallel machine scheduling problems was achieved. The presented ILS have found all the current Best Known Solutions in very competitive computational times for all presented instances. The sensitivity analyses obtained by testing the methods enabling and disabling neighborhoods and infeasibility strategies also showed which ones contribute more to the whole method and in which groups of instances they are more or less relevant.

As the results show, the infeasibility strategy does not seem to be very effective in the smaller instances, in the first four groups. Also, these strategies usually are more effective when the search goes on for a longer time. One recommended future work for this problem is to run the methods in more complex and larger instances, where probably the infeasible spaces will be better utilized. Generating these more complex instances would be a good contribution, since the PLSV instanes are limited to 50 operations so far.

Other neighborhoods could be implemented in our current Local Search and even other local search based metaheuristics can be simply coded from the presented work and to create a framework of metaheuristics for this problem is the next goal. Also it is possible to improve the neighborhood's efficiency by using pre-processing techniques, which could be an interesting future work.

The secondary objective of this thesis was also fulfilled as the instances were based on real-data from a studied company. The method was able to reach high quality solutions for those instances, indicating that it can maximize the company oil production. There are some limitations for this work, since this real-data-based instances have simulated attributes, so one possible future work is to test the method in the real-data and analyse the results to guide decision-making for the company.

As the problem described in this study is very particular, we limited our tests to the mentioned instances, but one possible future work is to test the method in other identical parallel machine scheduling problems, with similar but not equal problems characteristics. Probably some adaptations in the code would be necessary to adapt to the different characteristics, but it is a possible 
extension for this work.

About the problem, there are some attributes that were simplified or excluded from this thesis because they were not in the scope of our objectives. In this problem, we are not considering due dates for the operations, jobs and machines, but in the real-life problem application those are important factors. Also, we were aiming only to minimize weighted completion time, but the company is also interested in other objectives such as minimizing tardiness, minimizing fleet and others. The extension of this work to achieve these objectives would be a relevant possible future work.

At last, all of this thesis parameters were considered as deterministic. We know that in real-life applications, most of these parameters include uncertainty, specially in the operations processing times that depend on climate conditions, the operations release dates that depend on equipment arrival and environmental licenses, and the machines release dates depending on vessels contract features. Knowing that, one very relevant future work is proposing a stochastic version of this problem, including simulation methods to better estimate the mentioned attributes. 


\section{Bibliography}

Abu-Marrul, V., Martinelli, R., and Hamacher, S. (2019). Instances for the plsv scheduling problem. https://doi.org/10.17771/PUCRio.ResearchData.45799.

Abu-Marrul, V., Martinelli, R., and Hamacher, S. (2020a). Scheduling pipe laying support vessels with non-anticipatory family setup times and intersections between sets of operations. International Journal of Production Research, to appear.

Abu-Marrul, V., Mecler, D., Martinelli, R., Hamacher, S., and Gribkovskaia, I. (2020b). Heuristics for scheduling pipe-laying support vessels: An identical parallel machine scheduling approach. In 17th International Conference on Project Management and Scheduling.

Azizoglu, M. and Webster, S. (2003). Scheduling parallel machines to minimize weighted flowtime with family set-up times. International Journal of Production Research, 41(6):1199-1215.

Bremenkamp, L. H. (2017). Metodologia baseada em programação matemática para alocação e sequenciamento em embarcações plsv. Master's thesis, Departamento de Engenharia Industrial - Pontifícia Universidade Católica do Rio de Janeiro. in Portuguese.

Chen, Z.-L. and Powell, W. B. (2003). Exact algorithms for scheduling multiple families of jobs on parallel machines. Naval Research Logistics, 50(7):823-840.

Ciavotta, M., Meloni, C., and Pranzo, M. (2016). Speeding up a rollout algorithm for complex parallel machine scheduling. International Journal of Production Research, 54:4993-5009.

Dipak, L. and Gupta, J. N. D. (2018). An improved cuckoo search algorithm for scheduling jobs on identical parallel machines. Computers $\& 3$ Industrial Engineering, 126:348-360.

Dunstall, S. and Wirth, A. (2005a). A comparison of branch-and-bound algorithms for a family scheduling problem with identical parallel machines. European Journal of Operational Research, 167(2):283-296.

Dunstall, S. and Wirth, A. (2005b). Heuristic methods for the identical parallel machine flowtime problem with set-up times. Computers \& Operations Research, $32(9): 2479-2491$.

Kaplan, S. and Rabadi, G. (2011). Simulated annealing and metaheuristic for randomized priority search algorithms for the aerial refuelling parallel machine 
scheduling problem with due date-to-deadline windows and release times. Engineering Optimization, 45:67-87.

Kirkpatrick, S., Gelatt, C. D., and Vecchi, M. P. (1983). Optimization by simulated annealing. Science, 220(4598):671-680.

Lee, C. (2017). A dispatching rule and a random iterated greedy metaheuristic for identical parallel machine scheduling to minimize total tardiness. International Journal of Production Research, 56:1-17.

Liao, C.-J., Chao, C.-W., and Chen, L.-C. (2012). An improved heuristic for parallel machine weighted flowtime scheduling with family set-up times. Computers $\&$ Mathematics with Applications, 63(1):110-117.

Mehdizadeh, E., Tavakkoli-Moghaddam, R., and Yazdani, M. (2015). A vibration damping optimization algorithm for a parallel machines scheduling problem with sequence-independent family setup times. Applied Mathematical Modelling, $39(22): 6845-6859$.

Mendes, A. S.and Mueller, F. M., França, P. M., and Moscato, P. (2002). Comparing meta-heuristic approaches for parallel machine scheduling problems. Production Planning and Control, 13:143-154.

Min, L. and Cheng, W. (1999). A genetic algorithm for minimizing the makespan in the case of scheduling identical parallel machines. Artificial Intelligence in Engineering, 13:399-402.

Mokotoff, E. (2004). An exact algorithm for the identical parallel machine scheduling problem. European Journal of Operational Research, 152:758-769.

Nguyen, N., Yalaoui, F., Amodeo, L., C. H., and P., T. (2018). Total completion time minimization for machine scheduling problem under time windows constraints with jobs' linear processing rate function. Computers and Operations Research, 90:110-124.

Omar, M. K. and Teo, S. C. (2006). Minimizing the sum of earliness/tardiness in identical parallel machines schedule with incompatible job families: An improved MIP approach. Applied Mathematics and Computation, 181(2):1008-1017.

Pacciarelli, D., Meloni, C., and Pranzo, M. (2011). Models and methods for production scheduling in the pharmaceutical industry. International Series in Operations Research \&3 Management Science, 152:429-459.

Pisinger, D. and Røpke, S. (2007). A general heuristic for vehicle routing problems. Computers \& Operations Research, 34(8):2403-2435.

Potts, C. N. and Kovalyov, M. Y. (2000). Scheduling with batching: A review. European journal of operational research, 120(2):228-249.

Ruiz, R. and Maroto, C. (2005). An improved water flow-like algorithm for order acceptance and scheduling with identical parallel machines. European Journal of Operational Research, 165:479-494. 
Schaller, J. E. (2014). Minimizing total tardiness for scheduling identical parallel machines with family setups. Computers $\&$ Industrial Engineering, 72:274-281.

Shin, H. J. and Leon, V. J. (2004). International Journal of Production Research Scheduling with product family set-up times: an application in TFT LCD manufacturing Scheduling with product family set-up times: an application in TFT LCD manufacturing. International Journal of Production Research, 42(20):4235-4248.

Unlu, Y. and Mason, S. J. (2010). Evaluation of mixed integer programming formulations for non-preemptive parallel machine scheduling problems. Computers $\&$ Industrial Engineering, 58(4):785-800.

Van Der Zee, D.-J. (2015). Family-based dispatching with parallel machines. International Journal of Production Research, 53:5837-5856.

Webster, S. and Azizoglu, M. (2001). Dynamic programming algorithms for scheduling parallel machines with family setup times. Computers $\& 3$ Operations Research, 28(2):127-137.

Wu, G., Cheng, C., Yang, H., and Chena, C. (2018). A comprehensive review and evaluation of permutation flowshop heuristics. Applied Soft Computing, 71:1072-1084. 


\section{Detailed Results of the Methods for each Instance}

In this appendix, we present the complete results of our tests, that were summarized in Chapter 5. Table A.1 presents, for each instance and method, the main metrics that we defined to evaluate the results.

Since we run each method ten times, with ten different seeds, we gathered the minimum $\left(R P D^{-}\right)$, maximum $\left(R P D^{+}\right)$and average $(\overline{R P D}) R P D . R P D$ is computed as $R P D=\left(\frac{T W C-B K S}{B K S}\right) \times 100$. Where $T W C$ is the total weighted completion time obtained by the method in the specific instance and the $B K S$ is the best known solution. We present the $R P D$ standard deviation $\left(\sigma_{R P D}\right)$ and the $R P D_{L B}^{-}$computed as $R P D_{L B}^{-}=\left(\frac{L B-B K S}{B K S}\right) \times 100$, where $L B$ is the Lower Bound of the instance, indicating how far from the Lower Bound the method is in an specific instance. At last the $\overline{\text { Time }}$ is presented indicating the average computational time to run the method in each instance.

Table A.1: Complete Results for each Method and Instance

\begin{tabular}{lccccccc}
\hline Instance & Method & $R P D^{-}$ & $\overline{R P D}$ & $R P D^{+}$ & $\sigma_{R P D}$ & $R P D_{L B}^{-}$ & $\overline{T i m e}$ \\
\hline o15_n5_q3_m4_111 & ils & 0.000 & 0.000 & 0.000 & 0.000 & 0.000 & 0.60 \\
o15_n5_q3_m4_111 & ils-ni & 0.000 & 0.000 & 0.000 & 0.000 & 0.000 & 0.55 \\
o15_n5_q3_m4_111 & ils-ns & 0.000 & 0.000 & 0.000 & 0.000 & 0.000 & 0.34 \\
o15_n5_q3_m4_111 & ils-nr & 0.000 & 0.000 & 0.000 & 0.000 & 0.000 & 0.215 \\
o15_n5_q3_m4_111 & ils-nsi & 0.000 & 0.000 & 0.000 & 0.000 & 0.000 & 0.586 \\
o15_n5_q3_m4_111 & ils-nsr & 0.000 & 0.000 & 0.000 & 0.000 & 0.000 & 0.527 \\
o15_n5_q3_m4_111 & ils-os & 0.000 & 0.000 & 0.000 & 0.000 & 0.000 & 0.173 \\
o15_n5_q3_m4_111 & ils-or & 0.000 & 0.000 & 0.000 & 0.000 & 0.000 & 0.362 \\
o15_n5_q3_m4_112 & ils & 0.000 & 0.000 & 0.000 & 0.000 & 0.000 & 0.83 \\
o15_n5_q3_m4_112 & ils-ni & 0.000 & 0.000 & 0.000 & 0.000 & 0.000 & 0.80 \\
o15_n5_q3_m4_112 & ils-ns & 0.583 & 0.583 & 0.583 & 0.000 & 0.583 & 0.33 \\
o15_n5_q3_m4_112 & ils-nr & 0.000 & 0.238 & 0.596 & 23.754 & 0.000 & 0.281 \\
o15_n5_q3_m4_112 & ils-nsi & 0.000 & 0.000 & 0.000 & 0.000 & 0.000 & 0.752 \\
o15_n5_q3_m4_112 & ils-nsr & 0.000 & 0.000 & 0.000 & 0.000 & 0.000 & 0.767 \\
o15_n5_q3_m4_112 & ils-os & 0.000 & 0.555 & 1.076 & 31.975 & 0.000 & 0.239 \\
o15_n5_q3_m4_112 & ils-or & 0.583 & 0.583 & 0.583 & 0.000 & 0.583 & 0.359 \\
o15_n5_q3_m4_121 & ils & 0.000 & 0.000 & 0.000 & 0.000 & 0.000 & 0.62 \\
o15_n5_q3_m4_121 & ils-ni & 0.000 & 0.000 & 0.000 & 0.000 & 0.000 & 0.58 \\
\hline & & & & & Continued on & next & page
\end{tabular}




\begin{tabular}{|c|c|c|c|c|c|c|c|}
\hline Instance & Method & $R P D^{-}$ & $\overline{R P D}$ & $R P D^{+}$ & $\sigma_{R P D}$ & $R P D_{L B}^{-}$ & $\overline{\text { Time }}$ \\
\hline o15_n5_q3_m4 121 & ils-ns & 2.294 & 2.294 & 2.294 & 0.000 & 2.294 & 0.31 \\
\hline o15_n5_q3_m4_121 & ils-nr & 0.360 & 0.743 & 2.161 & 52.262 & 0.360 & 0.235 \\
\hline $\mathrm{o} 15 \_\mathrm{n} 5 \_\mathrm{q} 3 \_\mathrm{m} 4 \_121$ & ils-nsi & 0.000 & 0.000 & 0.000 & 0.000 & 0.000 & 0.569 \\
\hline o15_n5_q3_m4_121 & ils-nsr & 0.000 & 0.000 & 0.000 & 0.000 & 0.000 & 0.571 \\
\hline o15_n5_q3_m4_121 & ils-os & 0.360 & 2.082 & 3.241 & 87.708 & 0.360 & 0.199 \\
\hline o15_n5_q3_m4_121 & ils-or & 2.361 & 2.361 & 2.361 & 0.000 & 2.361 & 0.350 \\
\hline o15_n5_q3_m4_122 & ils & 0.000 & 0.000 & 0.000 & 0.000 & 0.000 & 0.68 \\
\hline o15_n5_q3_m4 122 & ils-ni & 0.000 & 0.000 & 0.000 & 0.000 & 0.000 & 0.70 \\
\hline o15_n5_q3_m4_122 & ils-ns & 5.797 & 5.797 & 5.797 & 0.000 & 5.797 & 0.31 \\
\hline o15_n5_q3_m4_122 & ils-nr & 0.836 & 2.104 & 5.658 & 141.002 & 0.836 & 0.244 \\
\hline o15_n5_q3_m4 122 & ils-nsi & 0.000 & 0.000 & 0.000 & 0.000 & 0.000 & 0.655 \\
\hline o15_n5_q3_m4_122 & ils-nsr & 0.000 & 0.000 & 0.000 & 0.000 & 0.000 & 0.645 \\
\hline o15_n5_q3_m4 122 & ils-os & 0.836 & 3.568 & 5.960 & 174.141 & 0.836 & 0.222 \\
\hline o15_n5_q3_m4_122 & ils-or & 5.797 & 5.797 & 5.797 & 0.000 & 5.797 & 0.372 \\
\hline o15_n5_q3_m4_131 & ils & 0.000 & 0.000 & 0.000 & 0.000 & 0.000 & 0.56 \\
\hline o15_n5_q3_m4_131 & ils-ni & 0.000 & 0.000 & 0.000 & 0.000 & 0.000 & 0.56 \\
\hline o15_n5_q3_m4_131 & ils-ns & 0.730 & 0.730 & 0.730 & 0.000 & 0.730 & 0.33 \\
\hline o15_n5_q3_m4 131 & ils-nr & 0.182 & 0.283 & 0.365 & 5.676 & 0.182 & 0.227 \\
\hline o15_n5_q3_m4_131 & ils-nsi & 0.000 & 0.000 & 0.000 & 0.000 & 0.000 & 0.548 \\
\hline o15_n5_q3_m4_131 & ils-nsr & 0.000 & 0.000 & 0.000 & 0.000 & 0.000 & 0.531 \\
\hline o15_n5_q3_m4_131 & ils-os & 0.365 & 0.520 & 1.186 & 35.917 & 0.365 & 0.163 \\
\hline o15_n5_q3_m4 131 & ils-or & 0.730 & 0.730 & 0.730 & 0.000 & 0.730 & 0.344 \\
\hline o15_n5_q3_m4 132 & ils & 0.000 & 0.000 & 0.000 & 0.000 & 0.000 & 0.76 \\
\hline o15_n5_q3_m4_132 & ils-ni & 0.000 & 0.000 & 0.000 & 0.000 & 0.000 & 0.66 \\
\hline o15_n5_q3_m4_132 & ils-ns & 5.793 & 5.793 & 5.793 & 0.000 & 5.793 & 0.41 \\
\hline o15_n5_q3_m4_132 & ils-nr & 0.000 & 0.000 & 0.000 & 0.000 & 0.000 & 0.273 \\
\hline o15_n5_q3_m4_132 & ils-nsi & 0.000 & 0.000 & 0.000 & 0.000 & 0.000 & 0.654 \\
\hline o15_n5_q3_m4 132 & ils-nsr & 0.000 & 0.000 & 0.000 & 0.000 & 0.000 & 0.658 \\
\hline o15_n5_q3_m4_132 & ils-os & 0.000 & 0.020 & 0.197 & 6.641 & 0.000 & 0.213 \\
\hline o15_n5_q3_m4_132 & ils-or & 4.689 & 4.689 & 4.689 & 0.000 & 4.689 & 0.343 \\
\hline o15_n5_q3_m4 211 & ils & 0.000 & 0.000 & 0.000 & 0.000 & 0.000 & 0.65 \\
\hline o15_n5_q3_m4 211 & ils-ni & 0.000 & 0.000 & 0.000 & 0.000 & 0.000 & 0.57 \\
\hline o15_n5_q3_m4 _211 & ils-ns & 4.886 & 4.886 & 4.886 & 0.000 & 4.886 & 0.47 \\
\hline o15_n5_q3_m4_211 & ils-nr & 0.000 & 0.173 & 1.734 & 48.383 & 0.000 & 0.233 \\
\hline o15_n5_q3_m4_211 & ils-nsi & 0.000 & 0.000 & 0.000 & 0.000 & 0.000 & 0.569 \\
\hline o15_n5_q3_m4_211 & ils-nsr & 0.000 & 0.000 & 0.000 & 0.000 & 0.000 & 0.541 \\
\hline o15_n5_q3_m4_211 & ils-os & 1.032 & 2.489 & 3.117 & 68.950 & 1.032 & 0.178 \\
\hline o15_n5_q3_m4 211 & ils-or & 4.886 & 4.886 & 4.886 & 0.000 & 4.886 & 0.390 \\
\hline o15_n5_q3_m4 212 & ils & 0.000 & 0.000 & 0.000 & 0.000 & 0.000 & 0.92 \\
\hline o15_n5_q3_m4_212 & ils-ni & 0.000 & 0.000 & 0.000 & 0.000 & 0.000 & 0.81 \\
\hline o15_n5_q3_m4_212 & ils-ns & 4.651 & 4.651 & 4.651 & 0.000 & 4.651 & 0.31 \\
\hline
\end{tabular}




\begin{tabular}{|c|c|c|c|c|c|c|c|}
\hline Instance & Method & $R P D^{-}$ & $\overline{R P D}$ & $R P D^{+}$ & $\sigma_{R P D}$ & $R P D_{L B}^{-}$ & $\overline{\text { Time }}$ \\
\hline o15_n5_q3_m4 & ils-nr & 0.106 & 0.106 & 0.106 & 0.000 & 0.106 & 0.315 \\
\hline o15_n5_q3_m4_212 & ils-nsi & 0.000 & 0.000 & 0.000 & 0.000 & 0.000 & 0.822 \\
\hline o15_n5_q3_m4 & ils-nsr & 0.000 & 0.000 & 0.000 & 0.000 & 0.000 & 0.823 \\
\hline o15_n5_q3_m4 & ils-os & 0.106 & 0.240 & 0.912 & 15.915 & 0.106 & 0.267 \\
\hline o15_n5_q3_m4 & ils-or & 4.651 & 4.651 & 4.651 & 0.000 & 4.651 & 0.337 \\
\hline o15_n5_q3_m4_221 & ils & 0.000 & 0.000 & 0.000 & 0.000 & 0.000 & 0.60 \\
\hline o15_n5_q3_m4 _221 & ils-ni & 0.000 & 0.000 & 0.000 & 0.000 & 00 & 0.70 \\
\hline o15_n5_q3_m4 & ils-ns & 2.193 & 2.193 & 2.193 & 0.000 & 193 & 0.34 \\
\hline o15_n5_q3_m4 & ils-nr & 0 & 0.000 & 0.000 & 0.0 & 000 & 0.237 \\
\hline o15_n5_q3_m4_221 & ils-nsi & 0.000 & 0.000 & 0.000 & 0.0 & 000 & 0.561 \\
\hline o15_n5_q3_m4 & ils-nsr & 0.000 & 0.000 & 0.000 & 0.000 & 000 & 0.635 \\
\hline o15_n5_q3_m4 & ils-os & 0.000 & 0.000 & 0.000 & 0.000 & 0.000 & 0.208 \\
\hline o15_n5_q3_m4 & ils-or & 2.193 & 2.193 & 2.193 & 0.000 & 2.193 & 0.361 \\
\hline o15_n5_q3_m4_222 & ils & 0.000 & 0.000 & 0.000 & 0.000 & 0.000 & 0.69 \\
\hline o15_n5_q3_m4 & ils-ni & 0.000 & 0.000 & 0.000 & 0.000 & 0.000 & 0.71 \\
\hline o15_n5_q3_m4 & ils-ns & 4.806 & 4.806 & 4.806 & 0.000 & 4.806 & 0.32 \\
\hline o15_n5_q3_m4_222 & ils-nr & 0.000 & 0.096 & 0.193 & 14.757 & 0.000 & 0.239 \\
\hline o15_n5_q3_m4_222 & ils-nsi & 0.000 & 0.000 & 0.000 & 0.000 & 0.000 & 0.592 \\
\hline o15_n5_q3_m4 & ils-nsr & 0.000 & 0.000 & 0.000 & 0.000 & 0.000 & 0.646 \\
\hline o15_n5_q3_m4 & ils- & 0 & 0.077 & 3 & 9 & 000 & 0.220 \\
\hline o15_n5_q3_m4_222 & ils-or & 4.806 & 6 & 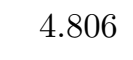 & & 6 & 0.341 \\
\hline o15_n5_q3_m4_231 & ils & 0 & 0.060 & 0.121 & & 0.000 & 0.68 \\
\hline o15_n5_q3_m4 & ils-ni & 0.000 & 0.036 & 0.121 & 6.280 & 0.000 & 0.78 \\
\hline o15_n5_q3_m4 & ils-ns & 5.253 & 5.253 & 5.253 & 0.000 & 5.263 & 0.32 \\
\hline o15_n5_q3_m4_231 & ils-nr & 0.195 & 0.195 & 0.195 & 0.000 & 0.205 & 0.249 \\
\hline o15_n5_q3_m4_231 & ils-nsi & 0.000 & 0.036 & 0.121 & 6.280 & 0.009 & 0.585 \\
\hline o15_n5_q3_m4 & ils-nsr & 0.000 & 0.012 & 0.121 & 4.111 & 0.009 & 0.644 \\
\hline o15_n5_q3_m4_231 & ils-os & 0.195 & 0.322 & 1.460 & 43.007 & 0.205 & 0.212 \\
\hline o15_n5_q3_m4_231 & ils-or & 5.904 & 5.904 & 5.904 & 10754. & 5.914 & 0.357 \\
\hline o15_n5_q3_m4 _232 & ils & 0 . & 0.000 & 0.000 & 0.000 & 0.000 & 0.56 \\
\hline o15_n5_q3_m4 & ils-ni & 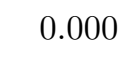 & 0.000 & 0.0 & 0.0 & 0.000 & 0.60 \\
\hline o15_n5_q3_m4_232 & ils-ns & 6 & 6.236 & 6.2 & O & 6.236 & 0.33 \\
\hline o15_n5_q3_m4_232 & ils-nr & 0.000 & 0.417 & 1.550 & 97.437 & 0.000 & 0.195 \\
\hline o15_n5_q3_m4 & ils-nsi & 0.000 & 0.000 & 0.000 & 0.000 & 0.000 & 0.585 \\
\hline o15_n5_q3_m4_232 & ils-nsr & 0.000 & 0.000 & 0.000 & 0.000 & 0.000 & 0.543 \\
\hline o15_n5_q3_m4_232 & ils-os & 0.000 & 1.243 & 2.737 & 162.076 & 0.000 & 0.175 \\
\hline o15_n5_q3_m4 _232 & ils-or & 6.236 & 6.236 & 6.236 & 0.000 & 6.236 & 0.310 \\
\hline o15_n5_q3_m8_111 & ils & 0.000 & 0.000 & 0.000 & 0.000 & 0.000 & 0.79 \\
\hline o15_n5_q3_m8_111 & ils-ni & 0.000 & 0.000 & 0.000 & 0.000 & 0.000 & 0.71 \\
\hline o15_n5_q3_m8_111 & ils-ns & 2.369 & 2.369 & 2.369 & 0.000 & 2.369 & 0.49 \\
\hline o15_n5_q3_m8_111 & ils-nr & 0.813 & 0.813 & 0.813 & 0.000 & 0.813 & 0.263 \\
\hline
\end{tabular}




\begin{tabular}{|c|c|c|c|c|c|c|c|}
\hline Instance & Method & $R P D^{-}$ & $\overline{R P D}$ & $R P D^{+}$ & $\sigma_{R P D}$ & $R P D_{L B}^{-}$ & $\overline{\text { Time }}$ \\
\hline o15_n5_q3_m8_111 & ils-nsi & 0.000 & 0.007 & 0.070 & 0.949 & 0.000 & 0.681 \\
\hline o15_n5_q3_m8_111 & ils-nsr & 0.000 & 0.000 & 0.000 & 0.000 & 0.000 & 0.698 \\
\hline o15_n5_q3_m8_111 & ils-os & 0.813 & 0.876 & 1.393 & 7.846 & 0.813 & 0.235 \\
\hline o15_n5_q3_m8_111 & ils-or & 2.508 & 2.508 & 2.508 & 0.000 & 2.508 & 0.392 \\
\hline o15_n5_q3_m8_112 & ils & 0.000 & 0.000 & 0.000 & 0.000 & 0.000 & 0.87 \\
\hline o15_n5_q3_m8_112 & ils-ni & 0.000 & 0.000 & 0.000 & 0.000 & 0.000 & 0.78 \\
\hline o15_n5_q3_m8_112 & ils-ns & 8.234 & 8.234 & 34 & 0.000 & 234 & 0.39 \\
\hline o15_n5_q3_m8_112 & ils-nr & 0.568 & 0.592 & 0.807 & 4.111 & 0.568 & 0.297 \\
\hline o15_n5_q3_m8_112 & ils-nsi & 0.000 & 0.000 & 0.000 & 0.000 & 0.000 & 0.712 \\
\hline o15_n5_q3_m8_112 & ils-nsr & 0.000 & 0.000 & 0.000 & 0.000 & 0.000 & 0.768 \\
\hline o15_n5_q3_m8_112 & ils-os & 1.064 & 1.097 & 1.284 & 3.706 & 1.064 & 0.246 \\
\hline o15_n5_q3_m8_112 & ils-or & 8.234 & 8.234 & 8.234 & 0.000 & 8.234 & 0.403 \\
\hline o15_n5_q3_m8_121 & ils & 0.000 & 0.000 & 0.000 & 0.000 & 0.000 & 0.65 \\
\hline o15_n5_q3_m8_121 & ils-ni & 0.000 & 0.000 & 0.000 & 0.000 & 0.000 & 0.63 \\
\hline o15_n5_q3_m8_121 & ils-ns & 0.000 & 0.000 & 0.000 & 0.000 & 0.000 & 0.46 \\
\hline o15_n5_q3_m8_121 & ils-nr & 0.000 & 0.000 & 0.000 & 0.000 & 0.000 & 0.258 \\
\hline o15_n5_q3_m8_121 & ils-nsi & 0.000 & 0.000 & 0.000 & 0.000 & 0.000 & 0.625 \\
\hline o15_n5_q3_m8_121 & ils-nsr & 0.000 & 0.000 & 0.000 & 0.000 & 0.000 & 0.537 \\
\hline o15_n5_q3_m8_121 & ils-os & 0.000 & 0.000 & 0.000 & 0.000 & 0.000 & 0.197 \\
\hline o15_n5_q3_m8_121 & ils-or & 0 & 0.000 & 00 & 0 & .000 & 0.379 \\
\hline o15_n5_q3_m8_122 & ils & 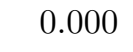 & 0.000 & 00 & 0.000 & 00 & 0.68 \\
\hline o15_n5_q3_m8_122 & ils-ni & 0 & 0.000 & 0 & 00 & 00 & 0.79 \\
\hline o15_n5_q3_m8_122 & ils-ns & 1.649 & 1.649 & 1.649 & 0.000 & 1.649 & 0.35 \\
\hline o15_n5_q3_m8_122 & ils-nr & 0.000 & 0.000 & 0.000 & 0.000 & 0.000 & 0.244 \\
\hline o15_n5_q3_m8_122 & ils-nsi & 0.000 & 0.000 & 0.000 & 0.000 & 0.000 & 0.653 \\
\hline o15_n5_q3_m8_122 & ils-nsr & 0.000 & 0.000 & 0.000 & 0.000 & 0.000 & 0.640 \\
\hline o15_n5_q3_m8_122 & ils-os & 0.000 & 0.000 & 0.000 & 0.000 & 0.000 & 0.233 \\
\hline o15_n5_q3_m8_122 & ils-or & 1.649 & 1.649 & 1.649 & 0.000 & 1.649 & 0.356 \\
\hline o15_n5_q3_m8_131 & ils & 0.000 & 0.318 & 3.180 & 43.639 & 0.000 & 0.71 \\
\hline o15_n5_q3_m8_131 & ils-ni & 0.000 & 0.028 & 0 & 2.530 & 0.000 & 0.69 \\
\hline o15_n5_q3_m8_131 & ils-ns & 0.042 & 3.042 & J. & 0.000 & 3.042 & 0.35 \\
\hline o15_n5_q3_m8_131 & ils-nr & 1.659 & 2.461 & 3 & 20.357 & 1.659 & 0.255 \\
\hline o15_n5_q3_m8_131 & ils-nsi & 0.000 & 0.014 & 0.138 & 1.897 & 0.000 & 0.653 \\
\hline o15_n5_q3_m8_131 & ils-nsr & 0.000 & 0.000 & 0.000 & 0.000 & 0.000 & 0.615 \\
\hline o15_n5_q3_m8_131 & ils-os & 2.627 & 2.627 & 2.627 & 0.000 & 2.627 & 0.205 \\
\hline o15_n5_q3_m8_131 & ils-or & 3.042 & 3.042 & 3.042 & 0.000 & 3.042 & 0.398 \\
\hline o15_n5_q3_m8_132 & ils & 0.000 & 0.015 & 0.149 & 3.479 & 0.000 & 0.73 \\
\hline o15_n5_q3_m8_132 & ils-ni & 0.000 & 0.000 & 0.000 & 0.000 & 0.000 & 0.87 \\
\hline o15_n5_q3_m8_132 & ils-ns & 1.465 & 1.465 & 1.465 & 0.000 & 1.465 & 0.41 \\
\hline o15_n5_q3_m8_132 & ils-nr & 0.109 & 0.396 & 0.760 & 20.060 & 0.109 & 0.245 \\
\hline o15_n5_q3_m8_132 & ils-nsi & 0.000 & 0.000 & 0.000 & 0.000 & 0.000 & 0.667 \\
\hline
\end{tabular}




\begin{tabular}{|c|c|c|c|c|c|c|c|}
\hline Instance & Method & $R P D^{-}$ & $\overline{R P D}$ & $R P D^{+}$ & $\sigma_{R P D}$ & $R P D_{L B}^{-}$ & $\overline{\text { Time }}$ \\
\hline o15_n5_q3_m8_132 & ils-nsr & 0.000 & 0.000 & 0.000 & 0.000 & 0.000 & 0.695 \\
\hline o15_n5_q3_m8_132 & ils-os & 0.109 & 0.355 & 0.760 & 21.994 & 0.109 & 0.216 \\
\hline o15_n5_q3_m8_132 & ils-or & 4.002 & 4.002 & 4.002 & 0.000 & 4.002 & 0.374 \\
\hline o15_n5_q3_m8_211 & ils & 0.000 & 0.000 & 0.000 & 0.000 & 0.000 & 0.79 \\
\hline o15_n5_q3_m8_211 & ils-ni & 0.000 & 0.000 & 0.000 & 0.000 & 0.000 & 0.83 \\
\hline o15_n5_q3_m8_211 & ils-ns & 1.631 & 1.631 & 1.631 & 0.000 & 1.631 & 0.35 \\
\hline o15_n5_q3_m8_211 & ils-nr & 0.533 & 0.561 & 0.564 & 0.316 & 0.533 & 0.293 \\
\hline o15_n5_q3_m8_211 & ils-nsi & 0.000 & 0.022 & 0.220 & 2.214 & 0.000 & 0.685 \\
\hline o15_n5_q3_m8_211 & ils-nsr & 0.000 & 0.022 & 20 & 2.214 & 0.000 & 0.735 \\
\hline o15_n5_q3_m8_211 & ils-os & 0.533 & 0.668 & 17 & 7.761 & 0.533 & 0.229 \\
\hline o15_n5_q3_m8_211 & ils-or & 1.380 & 1.380 & 1.380 & 0.000 & 1.380 & 0.372 \\
\hline o15_n5_q3_m8_212 & ils & 0.000 & 0.000 & 0.000 & 0.000 & 0.000 & 0.82 \\
\hline o15_n5_q3_m8_212 & ils-ni & 0.000 & 0.021 & 0.164 & 2.234 & 0.000 & 0.70 \\
\hline o15_n5_q3_m8_212 & ils-ns & 2.021 & 2.021 & 2.021 & 0.000 & 2.021 & 0.34 \\
\hline o15_n5_q3_m8_212 & ils-nr & 0.164 & 0.183 & 0.258 & 1.687 & 0.164 & 0.285 \\
\hline o15_n5_q3_m8_212 & ils-nsi & 0.000 & 0.049 & 0.164 & 3.381 & 0.000 & 0.701 \\
\hline o15_n5_q3_m8_212 & ils-nsr & 0.000 & 0.000 & 0.000 & 0.000 & 0.000 & 0.705 \\
\hline o15_n5_q3_m8_212 & ils-os & 0.164 & 0.174 & 58 & 1.265 & 0.164 & 0.230 \\
\hline o15_n5_q3_m8_212 & ils-or & 2.021 & 2.021 & 2.021 & 0.000 & 021 & 0.359 \\
\hline o15_n5_q3_m8_221 & ils & 0.000 & 1.058 & 2.174 & 44.109 & 0.000 & 0.87 \\
\hline o15_n5_q3_m8_221 & ils-ni & 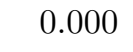 & 0.645 & 1 & 15 . & 000 & 0.78 \\
\hline o15_n5_q3_m8_221 & ils-ns & 3 & 1.703 & 2 & 0 & 03 & 0.41 \\
\hline o15_n5_q3_m8_221 & ils-nr & 0.580 & 1.410 & 2.537 & 33.072 & 0.580 & 0.291 \\
\hline o15_n5_q3_m8_221 & ils-nsi & 0.652 & 0.975 & 2.174 & 27.088 & 0.652 & 0.755 \\
\hline o15_n5_q3_m8_221 & ils-nsr & 0.000 & 0.743 & 2.174 & 33.879 & 0.000 & 0.670 \\
\hline o15_n5_q3_m8_221 & ils-os & 1.268 & 2.044 & 2.645 & 31.073 & 1.268 & 0.240 \\
\hline o15_n5_q3_m8_221 & ils-or & 2.790 & 2.790 & 2.790 & 0.000 & 2.790 & 0.412 \\
\hline o15_n5_q3_m8_222 & ils & 0.000 & 0.000 & 0.000 & 0.000 & 0.000 & 0.78 \\
\hline o15_n5_q3_m8_222 & ils-ni & 0.000 & 0.000 & 0.000 & 0.000 & 0.000 & 0.79 \\
\hline o15_n5_q3_m8_222 & ils-ns & 7.055 & 7.055 & 7.055 & 0.000 & 7.055 & 0.37 \\
\hline o15_n5_q3_m8_222 & ils-nr & 0.100 & 3.985 & 5.783 & 66.092 & 3.785 & 0.271 \\
\hline o15_n5_q3_m8_222 & ils-nsi & 0.000 & 0.000 & 0 & .000 & 0.000 & 0.607 \\
\hline o15_n5_q3_m8_222 & ils-nsr & 0.000 & 0.000 & 00 & 0.000 & 0.000 & 0.635 \\
\hline o15_n5_q3_m8_222 & ils-os & 3.785 & 3.879 & 4.722 & 30.990 & 3.785 & 0.232 \\
\hline o15_n5_q3_m8_222 & ils-or & 7.055 & 7.055 & 7.055 & 0.000 & 7.055 & 0.411 \\
\hline o15_n5_q3_m8_231 & ils & 0.000 & 0.019 & 0.187 & 4.743 & 0.000 & 0.76 \\
\hline o15_n5_q3_m8_231 & ils-ni & 0.000 & 0.037 & 0.187 & 6.325 & 0.000 & 0.78 \\
\hline o15_n5_q3_m8_231 & ils-ns & 0.337 & 0.337 & 0.337 & 0.000 & 0.337 & 0.32 \\
\hline o15_n5_q3_m8_231 & ils-nr & 0.187 & 0.247 & 0.337 & 5.138 & 0.187 & 0.250 \\
\hline o15_n5_q3_m8_231 & ils-nsi & 0.000 & 0.019 & 0.187 & 4.743 & 0.000 & 0.651 \\
\hline o15_n5_q3_m8_231 & ils-nsr & 0.000 & 0.000 & 0.000 & 0.000 & 0.000 & 0.690 \\
\hline
\end{tabular}




\begin{tabular}{|c|c|c|c|c|c|c|c|}
\hline Instance & Method & $R P D^{-}$ & $\overline{R P D}$ & $R P D^{+}$ & $\sigma_{R P D}$ & $R P D_{L B}^{-}$ & $\overline{\text { Time }}$ \\
\hline $\mathrm{o} 15 \_\mathrm{n} 5 \_\mathrm{q} 3 \_\mathrm{m} 8 \_231$ & ils-os & 0.187 & 0.304 & 0.412 & 6.075 & 0.187 & 0.218 \\
\hline o15_n5_q3_m8_231 & ils-or & 0.337 & 0.337 & 0.337 & 0.000 & 0.337 & 0.346 \\
\hline o15_n5_q3 $3 \_m 8 \_232$ & ils & 0.000 & 0.000 & 0.000 & 0.000 & 0.000 & 0.86 \\
\hline o15_n5_q3_m8_232 & ils-ni & 0.000 & 0.000 & 0.000 & 0.000 & 0.000 & 0.81 \\
\hline o15_n5_q3_m8_232 & ils-ns & 1.229 & 1.229 & 1.229 & 0.000 & 1.229 & 0.41 \\
\hline $\mathrm{o} 15 \_\mathrm{n} 5 \_\mathrm{q} 3 \_\mathrm{m} 8 \_232$ & ils-nr & 0.000 & 0.492 & 0.702 & 13.506 & 0.000 & 0.305 \\
\hline o15_n5_q3_m8_232 & ils-nsi & 0.000 & 0.000 & 0.000 & 0.000 & 0.000 & 0.753 \\
\hline o15_n5_q3_m8_232 & ils-nsr & 0.000 & 0.012 & 0.117 & 1.897 & 0.000 & 0.755 \\
\hline o15_n5_q3_m8_232 & ils-os & 0.000 & 0.427 & 0.819 & 19.081 & 0.000 & 0.265 \\
\hline o15_n5_q3_m8_232 & ils-or & 1.229 & 1.229 & 1.229 & 0.000 & 1.229 & 0.369 \\
\hline o25_n8_q3_m4_111 & ils & 0.000 & 0.000 & 0.000 & 0.000 & 2.566 & 2.85 \\
\hline o25_n8_q3_m4_111 & ils-ni & 0.000 & 0.000 & 0.000 & 0.000 & 2.566 & 2.78 \\
\hline $\mathrm{o} 25 \_\mathrm{n} 8 \_\mathrm{q} 3 \_\mathrm{m} 4 \_111$ & ils-ns & 2.874 & 2.874 & 2.874 & 0.000 & 5.513 & 1.07 \\
\hline o25_n8_q3_m4_111 & ils-nr & 0.579 & 0.766 & 1.038 & 12.758 & 3.160 & 0.845 \\
\hline o25_n8_q3_m4_111 & ils-nsi & 0.000 & 0.000 & 0.000 & 0.000 & 2.566 & 2.432 \\
\hline o $25 \_n 8 \_q 3 \_m 4 \_111$ & ils-nsr & 0.000 & 0.000 & 0.000 & 0.000 & 2.566 & 2.354 \\
\hline o25_n8_q3_m4_111 & ils-os & 0.710 & 0.989 & 1.519 & 19.884 & 3.294 & 0.793 \\
\hline $\mathrm{o} 25 \_n 8 \_\mathrm{q} 3 \_\mathrm{m} 4 \_111$ & ils-or & 2.907 & 2.907 & 2.907 & 0.000 & 5.547 & 1.103 \\
\hline o25_n8_q3_m4_112 & ils & -0.522 & -0.344 & -0.021 & 44.413 & 11.407 & 2.77 \\
\hline o25_n8_q3_m4_112 & ils-ni & -0.522 & -0.402 & 0.149 & 45.012 & 11.407 & 2.78 \\
\hline o25_n8_q3_m4_112 & ils-ns & 2.184 & 2.184 & 2.184 & 0.000 & 14.437 & 1.05 \\
\hline o $25 \_n 8 \_q 3 \_m 4 \_112$ & ils-nr & 0.202 & 0.398 & 0.996 & 51.998 & 12.219 & 1.055 \\
\hline o25_n8_q3_m4_112 & ils-nsi & -0.522 & -0.454 & -0.144 & 27.006 & 11.407 & 2.627 \\
\hline o25_n8_q3_m4_112 & ils-nsr & -0.522 & -0.492 & -0.224 & 17.709 & 11.407 & 2.608 \\
\hline o25_n8_q3_m4_112 & ils-os & 0.000 & 0.503 & 1.321 & 88.699 & 11.992 & 0.987 \\
\hline o25_n8_q3_m4_112 & ils-or & 1.912 & 1.912 & 1.912 & 0.000 & 14.133 & 1.104 \\
\hline o $25 \_n 8 \_q 3 \_m 4 \_121$ & ils & -1.163 & -1.098 & -0.514 & 47.434 & 5.485 & 3.48 \\
\hline $\mathrm{o} 25 \_\mathrm{n} 8 \_\mathrm{q} 3{ }_{3} \_\mathrm{m} 4 \_121$ & ils-ni & -1.163 & -1.043 & -0.514 & 58.836 & 5.485 & 3.39 \\
\hline o $25 \_n 8 \_q 3 \_m 4 \_121$ & ils-ns & 4.997 & 4.997 & 4.997 & 0.000 & 12.059 & 1.16 \\
\hline $\mathrm{o} 25 \_\mathrm{n} 8 \_\mathrm{q} 3 \_\mathrm{m} 4 \_121$ & ils-nr & -0.942 & -0.077 & 0.437 & 97.497 & 5.720 & 1.097 \\
\hline o25_n8_q3_m4_121 & ils-nsi & -1.163 & -1.012 & -0.380 & 73.639 & 5.485 & 3.369 \\
\hline $\mathrm{o} 25 \_\mathrm{n} 8 \_\mathrm{q} 3 \_\mathrm{m} 4 \_121$ & ils-nsr & -1.163 & -1.163 & -1.163 & 0.000 & 5.485 & 3.265 \\
\hline o $25 \_n 8 \_q 3 \_m 4 \_121$ & ils-os & -0.359 & -0.020 & 0.367 & 49.054 & 6.343 & 1.045 \\
\hline o $25 \_n 8 \_q 3 \_m 4 \_121$ & ils-or & 0.921 & 0.921 & 0.921 & 0.000 & 7.708 & 1.202 \\
\hline o25_n8_q3_m4_122 & ils & 0.000 & 0.000 & 0.000 & 0.000 & 3.640 & 3.03 \\
\hline o25_n8_q3_m4_122 & ils-ni & 0.000 & 0.000 & 0.000 & 0.000 & 3.640 & 2.67 \\
\hline $\mathrm{o} 25 \_\mathrm{n} 8 \_\mathrm{q} 3 \_\mathrm{m} 4 \_122$ & ils-ns & 2.803 & 2.803 & 2.803 & 0.000 & 6.545 & 1.07 \\
\hline o25_n8_q3_m4_122 & ils-nr & 0.081 & 0.371 & 1.804 & 67.899 & 3.723 & 0.870 \\
\hline o $25 \_n 8 \_q 3 \_m 4 \_122$ & ils-nsi & 0.000 & 0.000 & 0.000 & 0.000 & 3.640 & 2.953 \\
\hline o25_n8_q3_m4_122 & ils-nsr & 0.000 & 0.000 & 0.000 & 0.000 & 3.640 & 2.634 \\
\hline o25_n8_q3_m4 & ils-os & 0.081 & 0.239 & 0.814 & 27.475 & 3.723 & 0.779 \\
\hline
\end{tabular}




\begin{tabular}{|c|c|c|c|c|c|c|c|}
\hline Instance & Method & $R P D^{-}$ & $\overline{R P D}$ & $R P D^{+}$ & $\sigma_{R P D}$ & $R P D_{L B}^{-}$ & $\overline{\text { Time }}$ \\
\hline o $25 \_n 8 \_\mathrm{q} 3 \_m 4 \_122$ & ils-or & 2.642 & 2.642 & 2.642 & 0.000 & 6.378 & 1.158 \\
\hline o $25 \_n 8 \_q 3 \_m 4 \_131$ & ils & 0.061 & 0.149 & 0.165 & 11.474 & 4.402 & 3.19 \\
\hline o $25 \_n 8 \_q 3 \_m 4 \_131$ & ils-ni & 0.165 & 0.165 & 0.165 & 0.000 & 4.510 & 3.21 \\
\hline o $25 \_n 8 \_q 3 \_m 4 \_131$ & ils-ns & 3.171 & 3.171 & 3.171 & 0.000 & 7.647 & 1.22 \\
\hline o25_n8_q3_m4_131 & ils-nr & 0.317 & 0.571 & 0.841 & 50.213 & 4.669 & 1.000 \\
\hline o $25 \_n 8 \_q 3 \_m 4 \_131$ & ils-nsi & 0.113 & 0.159 & 0.165 & 5.376 & 4.456 & 3.063 \\
\hline o $25 \_n 8 \_q 3 \_m 4 \_131$ & ils-nsr & 0.061 & 0.149 & 0.165 & 11.474 & 4.402 & 3.054 \\
\hline o $25 \_n 8 \_q 3 \_m 4 \_131$ & ils-os & 0.549 & 0.778 & 1.003 & 50.553 & 4.911 & 0.932 \\
\hline o $25 \_n 8 \_q 3 \_m 4 \_131$ & ils-or & 3.171 & 3.171 & 3.171 & 0.000 & 7.647 & 1.214 \\
\hline o25_n8_q3_m4_132 & ils & 0.000 & 0.000 & 0.000 & 0.000 & 0.000 & 2.99 \\
\hline o25_n8_q3_m4_132 & ils-ni & 0.000 & 0.000 & 0.000 & 0.000 & 0.000 & 2.78 \\
\hline o $25 \_n 8 \_q 3 \_m 4 \_132$ & ils-ns & 1.085 & 1.085 & 1.085 & 0.000 & 1.085 & 1.22 \\
\hline o $25 \_n 8 \_q 3 \_m 4 \_132$ & ils-nr & 0.704 & 1.044 & 1.426 & 69.033 & 0.704 & 0.904 \\
\hline o $25 \_n 8 \_q 3 \_m 4 \_132$ & ils-nsi & 0.000 & 0.054 & 0.269 & 31.201 & 0.000 & 2.988 \\
\hline o25_n8_q3_m4_132 & ils-nsr & 0.000 & 0.081 & 0.269 & & 0.000 & 2.669 \\
\hline o25_n8_q3_m4_132 & ils-os & 0.704 & 1.259 & 1.764 & 117.384 & 0.704 & 0.815 \\
\hline o25_n8_q3_m4_132 & ils-or & 1.085 & 1.085 & 1.085 & 0.000 & 1.085 & 1.348 \\
\hline $\mathrm{o} 25 \_\mathrm{n} 8 \_\mathrm{q} 3 \_\mathrm{m} 4 \_211$ & ils & -1.392 & -1.392 & -1.392 & 0.000 & 9.972 & 2.58 \\
\hline o $25 \_n 8 \_q 3 \_m 4 \_211$ & ils-ni & -1.392 & -1.392 & -1.392 & 0.000 & 9.972 & 2.59 \\
\hline $\mathrm{o} 25 \_\mathrm{n} 8 \_\mathrm{q} 3 \_\mathrm{m} 4 \_211$ & ils-ns & 3.698 & 3.698 & 3.698 & 0.000 & 15.649 & 1.02 \\
\hline o $25 \_n 8 \_q 3 \_m 4 \_211$ & ils-nr & -1.392 & -1.065 & -0.206 & 141.346 & 9.972 & 0.985 \\
\hline o $25 \_n 8 \_q 3 \_m 4 \_211$ & ils-nsi & -1.392 & -1.392 & -1.392 & 0.000 & 9.972 & 2.404 \\
\hline o $25 \_n 8 \_q 3 \_m 4 \_211$ & ils-nsr & -1.392 & -1.392 & -1.392 & 0.000 & 9.972 & 2.437 \\
\hline o $25 \_n 8 \_q 3 \_m 4 \_211$ & ils-os & -1.392 & -0.981 & -0.299 & 128.902 & 9.972 & 0.951 \\
\hline $\mathrm{o} 25 \_\mathrm{n} 8 \_\mathrm{q} 3 \_\mathrm{m} 4 \_211$ & ils-or & 3.698 & 3.698 & 3.698 & 0.000 & 15.649 & 1.017 \\
\hline $\mathrm{o} 25 \_\mathrm{n} 8 \_\mathrm{q} 3 \_\mathrm{m} 4 \_212$ & ils & -2.084 & -2.084 & -2.084 & 0.000 & 10.945 & 2.99 \\
\hline o $25 \_n 8 \_q 3 \_m 4 \_212$ & ils-ni & -2.084 & -2.074 & -2.024 & 4.175 & 10.945 & 3.19 \\
\hline o $25 \_n 8 \_q 3 \_m 4 \_212$ & ils-ns & 5.277 & 5.277 & 5.277 & 0.000 & 19.285 & 1.13 \\
\hline o $25 \_n 8 \_q 3 \_m 4 \_212$ & ils-nr & -2.084 & -1.622 & -0.944 & 60.178 & 10.945 & 0.944 \\
\hline o $25 \_n 8 \_q 3 \_m 4 \_212$ & ils-nsi & -2.084 & -2.078 & -2.024 & 3.795 & 10.945 & 2.822 \\
\hline o $25 \_n 8 \_q 3 \_m 4 \_212$ & ils-nsr & -2.084 & -2.084 & -2.084 & 0.000 & 10.945 & 2.785 \\
\hline o $25 \_n 8 \_q 3 \_m 4 \_212$ & ils-os & -1.679 & -1.295 & -0.515 & 78.266 & 11.404 & 0.903 \\
\hline o $25 \_n 8 \_\mathrm{q} 3 \_\mathrm{m} 4 \_212$ & ils-or & 4.922 & 4.922 & 4.922 & 0.000 & 18.883 & 1.182 \\
\hline o $25 \_n 8 \_q 3 \_m 4 \_221$ & ils & -0.557 & -0.557 & -0.557 & 0.000 & 4.360 & 2.81 \\
\hline o $25 \_n 8 \_q 3 \_m 4 \_221$ & ils-ni & -0.557 & -0.557 & -0.557 & 0.000 & 4.360 & 2.73 \\
\hline o25_n8_q3_m4_221 & ils-ns & 4.954 & 4.954 & 4.954 & 0.000 & 10.143 & 1.06 \\
\hline o $25 \_n 8 \_q 3 \_m 4 \_221$ & ils-nr & 0.481 & 0.656 & 0.847 & 25.963 & 5.449 & 0.920 \\
\hline o $25 \_n 8 \_q 3 \_m 4 \_221$ & ils-nsi & -0.557 & -0.557 & -0.557 & 0.000 & 4.360 & 2.647 \\
\hline o $25 \_n 8 \_\mathrm{q} 3 \_\mathrm{m} 4 \_221$ & ils-nsr & -0.557 & -0.557 & -0.557 & 0.000 & 4.360 & 2.660 \\
\hline o25_n8_q3_m4_221 & ils-os & 0.461 & 0.918 & 1.218 & 50.342 & 5.428 & 0.910 \\
\hline o $25 \_n 8 \_q 3 \_m 4 \_221$ & ils-or & 5.159 & 5.159 & 5.159 & 0.000 & 10.359 & 1.198 \\
\hline
\end{tabular}




\begin{tabular}{|c|c|c|c|c|c|c|c|}
\hline Instance & Method & $R P D^{-}$ & $\overline{R P D}$ & $R P D^{+}$ & $R P D$ & $R P D_{L B}^{-}$ & $\overline{\text { Time }}$ \\
\hline o25_n8_q3_m4_222 & ils & -0.462 & -0.423 & -0.326 & 12.186 & 10.799 & 2.51 \\
\hline o25_n8_q3 $3 \_m 4 \_222$ & ils-ni & -0.462 & -0.407 & -0.396 & 6.332 & 10.799 & 2.36 \\
\hline o25_n8_q3_m4_222 & ils-ns & 0.110 & 0.110 & 0.110 & 0.000 & 11.436 & 1.11 \\
\hline o25_n8_q3_m4_222 & ils-nr & -0.326 & 0.085 & 0.480 & 63.699 & 10.950 & 0.759 \\
\hline o25_n8_q3_m4 2222 & ils-nsi & -0.396 & -0.396 & -0.396 & 0.000 & 10.873 & 2.315 \\
\hline o25_n8_q3 $3 \_m 4 \_222$ & ils-nsr & -0.462 & -0.417 & -0.396 & 7.843 & 10.799 & 2.215 \\
\hline o25_n8_q3_m4 2222 & ils-os & -0.044 & 0.261 & 0.579 & 58.581 & 11.264 & 0.691 \\
\hline o25_n8_q3_m4_222 & ils-or & 0.110 & 0.110 & 10 & 0.000 & 11.436 & 1.163 \\
\hline o25_n8_q3_m4_231 & ils & .000 & 0.000 & 0 & 00 & 225 & 2.98 \\
\hline o25_n8_q3_m4_231 & ils-ni & 0.000 & 0.000 & 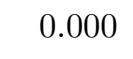 & 0.000 & 225 & 2.58 \\
\hline o25_n8_q3_m4_231 & ils-ns & 4.199 & 4.199 & 199 & 0.000 & 6.518 & 1.01 \\
\hline o25_n8_q3_m4_231 & ils-nr & 0.491 & 1.666 & 2.883 & 223.599 & 2.727 & 0.830 \\
\hline o25_n8_q3_m4_231 & ils-nsi & 0.000 & 0.000 & 0.000 & 0.000 & 2.225 & 2.582 \\
\hline o25_n8_q3_m4 _231 & ils-nsr & 0.000 & 0.000 & 0.000 & 0.000 & 2.225 & 2.499 \\
\hline o25_n8_q3_m4_231 & ils-os & 1.282 & 1.932 & 2.555 & 119.380 & 3.536 & 0.792 \\
\hline o25_n8_q3_m4_231 & ils-or & 4.199 & 4.199 & 4.199 & 0.000 & 6.518 & 1.066 \\
\hline o25_n8_q3_m4 232 & ils & -0.403 & -0.355 & 0.078 & 44.904 & 5.411 & 3.16 \\
\hline o25_n8_q3_m4 232 & ils-ni & -0.403 & -0.385 & -0.217 & 17.393 & 5.411 & 3.02 \\
\hline o25_n8_q3_m4_232 & ils-ns & 169 & 3.169 & 169 & 0.000 & .192 & 1.15 \\
\hline o25_n8_q3_m4 232 & ils- & 14 & 0.086 & $x$ & 11 & 791 & 1.010 \\
\hline o25_n8_q3_m4_232 & il & 3 & -0 & 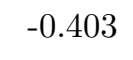 & 0 & 1 & 2.969 \\
\hline o25_n8_q3_m4 232 & ils-nsr & 3 & -0.403 & 12 & 0 & & 2.949 \\
\hline o25_n8_q3_m4 232 & ils-os & 0.075 & 0.273 & 1.112 & 90.068 & 5.917 & 0.985 \\
\hline o25_n8_q3 $3 \_m 4 \_232$ & ils-or & 3.417 & 3.417 & 3.417 & 0.000 & 9.454 & 1.207 \\
\hline o25_n8_q3_m8_111 & ils & -1.133 & -0.842 & -0.260 & 33.116 & 4.021 & 3.36 \\
\hline o25_n8_q3_m8_111 & ils-ni & -0.926 & -0.676 & -0.398 & 26.104 & 4.239 & 3.30 \\
\hline o25_n8_q3_m8_111 & ils-ns & 2.457 & 2.457 & 2.457 & 0.000 & 7.799 & 1.26 \\
\hline o25_n8_q3_m8_111 & ils-nr & -0.813 & 0.139 & 0.986 & 62.310 & 4.358 & 1.019 \\
\hline o25_n8_q3_m8_111 & ils- & -1 & -0.928 & 8 & & 557 & 3.160 \\
\hline o25_n8_q3_m8_111 & ils-nsr & -0.986 & -0.824 & -0.424 & 22.394 & 4.176 & 3.006 \\
\hline o25_n8_q3_m8_111 & ils-os & 0.407 & 0.716 & & 25.024 & 5.641 & 0.887 \\
\hline o25_n8_q3_m8_111 & ils-or & 3.971 & 3.971 & & 0.000 & 9.392 & 1.231 \\
\hline o25_n8_q3_m8_112 & ils & -0.601 & -0.539 & -0.272 & 15.720 & 6.724 & 3.67 \\
\hline o25_n8_q3_m8_112 & ils-ni & -0.601 & -0.394 & -0.012 & 28.001 & 6.724 & 3.47 \\
\hline o25_n8_q3_m8_112 & ils-ns & 6.520 & 6.520 & 6.520 & 0.000 & 14.369 & 1.31 \\
\hline o25_n8_q3_m8_112 & ils-nr & 2.322 & 2.676 & 3.077 & 36.907 & 9.862 & 1.220 \\
\hline o25_n8_q3_m8_112 & ils-nsi & -0.570 & -0.443 & -0.272 & 20.951 & 6.757 & 3.554 \\
\hline o25_n8_q3_m8_112 & ils-nsr & -0.570 & -0.430 & -0.272 & 20.024 & 6.757 & 3.457 \\
\hline o25_n8_q3_m8_112 & ils-os & 2.050 & 2.731 & 3.505 & 63.757 & 9.569 & 1.129 \\
\hline o25_n8_q3_m8_112 & ils-or & 6.811 & 6.811 & 6.811 & 0.000 & 14.682 & 1.388 \\
\hline o25_n8_q3_m8_121 & ils & 0.000 & 0.408 & 0.697 & 31.590 & 0.593 & 3.30 \\
\hline
\end{tabular}




\begin{tabular}{|c|c|c|c|c|c|c|c|}
\hline Instance & Method & $R P D^{-}$ & $\overline{R P D}$ & $R P D^{+}$ & $\sigma_{R P D}$ & $R P D_{L B}^{-}$ & $\overline{\text { Time }}$ \\
\hline o25_n8_q3_m8_121 & ils-ni & 0.105 & 0.459 & 0.678 & 21.419 & 0.699 & 3.21 \\
\hline o $25 \_n 8 \_q 3 \_m 8 \_121$ & ils-ns & 4.963 & 4.963 & 4.963 & 0.000 & 5.586 & 1.45 \\
\hline o $25 \_n 8 \_q 3 \_m 8 \_121$ & ils-nr & 0.687 & 1.206 & 1.642 & 33.791 & 1.285 & 0.998 \\
\hline o $25 \_n 8 \_q 3 \_m 8 \_121$ & ils-nsi & 0.000 & 0.453 & 0.783 & 29.224 & 0.593 & 3.055 \\
\hline o25_n8_q3_m8_121 & ils-nsr & 0.000 & 0.440 & 0.802 & 33.838 & 0.593 & 3.010 \\
\hline o $25 \_n 8 \_q 3 \_m 8 \_121$ & ils-os & 1.012 & 1.219 & 1.727 & 22.804 & 1.611 & 0.933 \\
\hline o $25 \_n 8 \_q 3 \_m 8 \_121$ & ils-or & 4.648 & 4.648 & 4.648 & 0.000 & 5.269 & 1.411 \\
\hline o $25 \_n 8 \_q 3 \_m 8 \_122$ & ils & -0.385 & -0.271 & 0.096 & 30.945 & 7.046 & 2.69 \\
\hline o $25 \_n 8 \_q 3 \_m 8 \_122$ & ils-ni & -0.254 & -0.038 & 0.223 & 42.053 & 7.188 & 2.54 \\
\hline o25_n8_q3_m8_122 & ils-ns & 3.443 & 3.443 & 3.443 & 0.000 & 11.160 & 1.30 \\
\hline o25_n8_q3_m8_122 & ils-nr & 0.482 & 1.206 & 1.795 & 71.879 & 7.978 & 0.882 \\
\hline o $25 \_n 8 \_q 3 \_m 8 \_122$ & ils-nsi & -0.385 & -0.161 & 0.304 & 49.313 & 7.046 & 2.505 \\
\hline o $25 \_n 8 \_q 3 \_m 8 \_122$ & ils-nsr & -0.385 & -0.257 & -0.157 & 16.248 & 7.046 & 2.476 \\
\hline o $25 \_n 8 \_q 3 \_m 8 \_122$ & ils-os & 1.197 & 1.692 & 2.287 & 78.940 & 8.746 & 0.770 \\
\hline o $25 \_n 8 \_q 3 \_m 8 \_122$ & ils-or & 6.074 & 6.074 & 6.074 & 0.000 & 13.987 & 1.270 \\
\hline o25_n8_q3_m8_131 & ils & 0.000 & 0.000 & 0.000 & 0.000 & 0.002 & 2.89 \\
\hline o25_n8_q3_m8_131 & ils-ni & 0.000 & 0.029 & 0.165 & 5.266 & 0.002 & 2.87 \\
\hline o $25 \_n 8 \_q 3 \_m 8 \_131$ & ils-ns & 3.691 & 3.691 & 3.691 & 0.000 & 3.693 & 1.25 \\
\hline o $25 \_n 8 \_q 3 \_m 8 \_131$ & ils-nr & 0.062 & 0.300 & 0.619 & 16.374 & 0.064 & 0.940 \\
\hline o25_n8_q3_m8_131 & ils-nsi & 0.000 & 0.025 & 0.186 & & 0.002 & 2.794 \\
\hline o25_n8_q3_m8_131 & ils-nsr & 0.000 & 0.000 & 0.000 & 0.000 & 0.002 & 2.676 \\
\hline o $25 \_n 8 \_q 3 \_m 8 \_131$ & ils-os & 0.062 & 0.524 & 0.835 & 28.055 & 0.064 & 0.884 \\
\hline o $25 \_n 8 \_q 3 \_m 8 \_131$ & ils-or & 3.691 & 3.691 & 3.691 & 0.000 & 3.693 & 1.338 \\
\hline o $25 \_n 8 \_q 3 \_m 8 \_132$ & ils & -0.201 & -0.098 & 0.234 & 27.097 & 1.687 & 3.26 \\
\hline o $25 \_n 8 \_q 3 \_m 8 \_132$ & ils-ni & -0.201 & -0.154 & 0.167 & 20.966 & 1.687 & 3.16 \\
\hline o25_n8_q3_m8_132 & ils-ns & 5.121 & 5.121 & 5.121 & 0.000 & 7.109 & 1.27 \\
\hline o25_n8_q3_m8_132 & ils-nr & 0.568 & 1.023 & 1.872 & 80.359 & 2.471 & 1.029 \\
\hline o $25 \_n 8 \_q 3 \_m 8 \_132$ & ils-nsi & -0.201 & -0.156 & 0.084 & 16.234 & 1.687 & 3.236 \\
\hline o $25 \_n 8 \_q 3 \_m 8 \_132$ & ils-nsr & -0.201 & -0.178 & -0.095 & 7.505 & 1.687 & 2.997 \\
\hline o25_n8_q3_m8_132 & ils-os & 0.435 & 1.026 & 1.543 & 63.877 & 2.335 & 0.984 \\
\hline o25_n8_q3_m8_132 & ils-or & 5.121 & 5.121 & 5.121 & 0.000 & 7.109 & 1.238 \\
\hline o $25 \_n 8 \_q 3 \_m 8 \_211$ & ils & -1.290 & -1.057 & -0.824 & 17.797 & 2.774 & 3.72 \\
\hline o $25 \_n 8 \_q 3 \_m 8 \_211$ & ils-ni & -0.968 & -0.780 & -0.466 & 13.937 & 3.110 & 3.87 \\
\hline o $25 \_n 8 \_q 3 \_m 8 \_211$ & ils-ns & 6.046 & 6.046 & 6.046 & 0.000 & 10.413 & 1.25 \\
\hline o $25 \_n 8 \_q 3 \_m 8 \_211$ & ils-nr & -0.633 & -0.179 & 0.167 & 24.345 & 3.459 & 1.109 \\
\hline o25_n8_q3_m8_211 & ils-nsi & -1.290 & -1.036 & -0.824 & 15.195 & 2.774 & 3.735 \\
\hline o $25 \_n 8 \_q 3 \_m 8 \_211$ & ils-nsr & -1.290 & -0.950 & -0.824 & 10.947 & 2.774 & 3.613 \\
\hline o $25 \_n 8 \_q 3 \_m 8 \_211$ & ils-os & -0.203 & 0.103 & 0.609 & 20.738 & 3.906 & 1.040 \\
\hline o $25 \_n 8 \_q 3 \_m 8 \_211$ & ils-or & 6.022 & 6.022 & 6.022 & 0.000 & 10.388 & 1.181 \\
\hline o25_n8_q3_m8_212 & ils & -1.339 & -0.854 & -0.303 & 47.778 & 4.675 & 3.28 \\
\hline o $25 \_n 8 \_q 3 \_m 8 \_212$ & ils-ni & -0.962 & -0.765 & -0.215 & 39.981 & 5.076 & 3.44 \\
\hline
\end{tabular}




\begin{tabular}{|c|c|c|c|c|c|c|c|}
\hline Instance & Method & $R P D^{-}$ & $\overline{R P D}$ & $R P D^{+}$ & $\sigma_{R P D}$ & $R P D_{L B}^{-}$ & $\overline{\text { Time }}$ \\
\hline o25_n8_q3_m8_212 & ils-ns & 3.632 & 3.632 & 3.632 & 0.000 & 9.950 & 1.21 \\
\hline o25_n8_q3_m8_212 & ils-nr & -0.503 & 0.139 & 0.481 & 45.672 & 5.562 & 1.123 \\
\hline o25_n8_q3_m8_212 & ils-nsi & -1.339 & -0.862 & 0.222 & 54.801 & 4.675 & 3.197 \\
\hline o25_n8_q3 $3 \_m 8 \_212$ & ils-nsr & -1.339 & -0.754 & -0.333 & 45.379 & 4.675 & 3.225 \\
\hline o $25 \_n 8 \_q 3 \_m 8 \_212$ & ils-os & -0.311 & 0.432 & 1.280 & 76.757 & 5.766 & 1.052 \\
\hline o25_n8_q3 $3 \_m 8 \_212$ & ils-or & 3.795 & 3.795 & 3.795 & 0.000 & 10.122 & 1.146 \\
\hline o $25 \_n 8 \_q 3 \_m 8 \_221$ & ils & 0.000 & 0.135 & 0.946 & 31.815 & 0.180 & 3.08 \\
\hline o25_n8_q3 $3 \_m 8 \_221$ & ils-ni & 0.000 & 0.030 & 0.100 & 5.314 & 0.180 & 2.89 \\
\hline o $25 \_n 8 \_q 3 \_m 8 \_221$ & ils-ns & 3.129 & 3.129 & 3.129 & 0.000 & 3.314 & 1.22 \\
\hline o $25 \_n 8 \_q 3 \_m 8 \_221$ & ils-nr & 1.992 & 2.284 & 2.984 & 33.238 & 2.175 & 0.910 \\
\hline o25_n8_q3 $3 \_m 8 \_221$ & ils-nsi & 0.000 & 0.050 & 0.100 & 5.798 & 0.180 & 2.816 \\
\hline o $25 \_n 8 \_q 3 \_m 8 \_221$ & ils-nsr & 0.000 & 0.040 & 0.100 & 5.680 & 0.180 & 2.745 \\
\hline o25_n8_q3_m8_221 & ils-os & 1.856 & 2.552 & 3.248 & 53.910 & 2.039 & 0.818 \\
\hline o $25 \_n 8 \_q 3 \_m 8 \_221$ & ils-or & 3.921 & 3.921 & 3.921 & 0.000 & 4.107 & 1.379 \\
\hline o $25 \_n 8 \_q 3 \_m 8 \_222$ & ils & -2.362 & -2.176 & -1.881 & 28.663 & 6.170 & 3.86 \\
\hline o25_n8_q3 $3 \_m 8 \_222$ & ils-ni & -2.362 & -2.207 & -1.959 & 26.232 & 6.170 & 3.60 \\
\hline o $25 \_n 8 \_q 3 \_m 8 \_222$ & ils-ns & 5.813 & 5.813 & 5.813 & 0.000 & 15.060 & 1.37 \\
\hline o25_n8_q3 $3 \_m 8 \_222$ & ils-nr & -1.938 & -1.491 & -1.089 & 40.625 & 6.631 & 1.215 \\
\hline o $25 \_n 8 \_q 3 \_m 8 \_222$ & ils-nsi & -2.362 & -2.227 & -1.669 & 35.716 & 6.170 & 3.567 \\
\hline o $25 \_n 8 \_q 3 \_m 8 \_222$ & ils-nsr & -2.362 & -2.171 & -1.761 & 31.774 & 6.170 & 3.569 \\
\hline o25_n8_q3 $3 \_m 8 \_222$ & ils-os & -1.938 & -1.332 & -0.806 & 53.877 & 6.631 & 1.167 \\
\hline o $25 \_n 8 \_q 3 \_m 8 \_222$ & ils-or & 4.958 & 4.958 & 4.958 & 0.000 & 14.129 & 1.398 \\
\hline o25_n8_q3 $3 \_m 8 \_231$ & ils & -0.227 & -0.201 & 0.032 & 7.589 & 1.295 & 3.15 \\
\hline o $25 \_n 8 \_q 3 \_m 8 \_231$ & ils-ni & -0.227 & -0.227 & -0.227 & 0.000 & 1.295 & 2.96 \\
\hline o $25 \_n 8 \_q 3 \_m 8 \_231$ & ils-ns & 2.151 & 2.151 & 2.151 & 0.000 & 3.709 & 1.25 \\
\hline o $25 \_n 8 \_q 3 \_m 8 \_231$ & ils-nr & 0.303 & 0.500 & 0.681 & 13.704 & 1.833 & 1.014 \\
\hline o25_n8_q3 $3 \_m 8 \_231$ & ils-nsi & -0.227 & -0.227 & -0.227 & 0.000 & 1.295 & 3.003 \\
\hline o25_n8_q3 $3 \_m 8 \_231$ & ils-nsr & -0.227 & -0.227 & -0.227 & 0.000 & 1.295 & 2.991 \\
\hline o $25 \_n 8 \_q 3 \_m 8 \_231$ & ils-os & 0.540 & 0.715 & 0.940 & 11.070 & 2.074 & 0.965 \\
\hline o $25 \_n 8 \_q 3 \_m 8 \_231$ & ils-or & 2.054 & 2.054 & 2.054 & 0.000 & 3.611 & 1.231 \\
\hline o25_n8_q3 $3 \_m 8 \_232$ & ils & -0.366 & -0.184 & -0.059 & 15.526 & 2.657 & 3.64 \\
\hline o25_n8_q3 $3 \_m 8 \_232$ & ils-ni & -0.289 & -0.181 & -0.065 & 12.240 & 2.736 & 3.50 \\
\hline o25_n8_q3 $3 \_m 8 \_232$ & ils-ns & 3.402 & 3.402 & 3.402 & 0.000 & 6.540 & 1.32 \\
\hline o $25 \_n 8 \_q 3 \_m 8 \_232$ & ils-nr & 0.053 & 0.245 & 0.396 & 19.783 & 3.089 & 1.124 \\
\hline o $25 \_n 8 \_q 3 \_m 8 \_232$ & ils-nsi & -0.366 & -0.208 & -0.083 & 17.041 & 2.657 & 3.446 \\
\hline o $25 \_n 8 \_q 3 \_m 8 \_232$ & ils-nsr & -0.390 & -0.177 & -0.083 & 16.234 & 2.633 & 3.337 \\
\hline o $25 \_n 8 \_q 3 \_m 8 \_232$ & ils-os & 0.159 & 0.226 & 0.425 & 13.425 & 3.199 & 1.066 \\
\hline o25_n8_q3 $3 \_m 8 \_232$ & ils-or & 3.208 & 3.208 & 3.208 & 0.000 & 6.339 & 1.387 \\
\hline $\mathrm{o} 50 \_\mathrm{n} 16 \_\mathrm{q} 3 \_\mathrm{m} 4 \_111$ & ils & -4.932 & -4.656 & -4.366 & 125.615 & 13.851 & 44.10 \\
\hline $\mathrm{o} 50 \_\mathrm{n} 16 \_\mathrm{q} 3 \_\mathrm{m} 4 \_111$ & ils-ni & -5.024 & -4.597 & -4.080 & 171.786 & 13.741 & 35.20 \\
\hline $\mathrm{o} 50 \_\mathrm{n} 16 \_\mathrm{q} 3 \_\mathrm{m} 4 \_111$ & ils-ns & 4.239 & 4.239 & 4.239 & 0.000 & 24.835 & 6.40 \\
\hline
\end{tabular}




\begin{tabular}{|c|c|c|c|c|c|c|c|}
\hline Instance & Method & $R P D^{-}$ & $\overline{R P D}$ & $R P D^{+}$ & $\sigma_{R P D}$ & $R P D_{L B}^{-}$ & $\overline{\text { Time }}$ \\
\hline o50_n16_q3_m4_111 & ils-nr & -3.877 & -3.142 & -2.284 & 389.740 & 15.115 & 11.535 \\
\hline o50_n16_q3_m4_111 & ils-nsi & -4.834 & -4.585 & -4.286 & 128.645 & 13.969 & 40.604 \\
\hline o50_n16_q3_m4_111 & ils-nsr & -5.012 & -4.622 & -4.414 & 106.700 & 13.756 & 42.881 \\
\hline o50_n16_q3_m4_111 & ils-os & -3.746 & -3.130 & -2.346 & 283.493 & 15.272 & 12.612 \\
\hline $\mathrm{o} 50 \_\mathrm{n} 16 \_\mathrm{q} 3 \_\mathrm{m} 4 \_111$ & ils-or & 4.303 & 4.303 & 4.303 & 0.000 & 24.911 & 7.127 \\
\hline o50_n16_q3_m4_112 & ils & -4.835 & -4.394 & -4.171 & 197.004 & 18.841 & 34.94 \\
\hline o50_n16_q3_m4_112 & ils-ni & -4.382 & -4.078 & -3.619 & 233.386 & 19.407 & 26.96 \\
\hline o50_n16_q3_m4_112 & ils-ns & 0.200 & 0.200 & 0.200 & 0.000 & 25.128 & 7.61 \\
\hline o50_n16_q3_m4_112 & ils-nr & -3.786 & -2.720 & -1.912 & 571.159 & 20.151 & 9.221 \\
\hline o50_n16_q3_m4_112 & ils-nsi & -4.473 & -4.193 & -3.874 & 161.492 & 19.294 & 33.063 \\
\hline o50_n16_q3_m4_112 & ils-nsr & -4.876 & -4.398 & -3.810 & 311.662 & 18.789 & 32.452 \\
\hline o50_n16_q3_m4_112 & ils-os & -3.877 & -2.362 & -1.727 & 589.493 & 20.038 & 10.045 \\
\hline o50_n16_q3_m4_112 & ils-or & 1.024 & 1.024 & 1.024 & 0.000 & 26.158 & 8.334 \\
\hline o50_n16_q3_m4_121 & ils & -2.727 & -2.551 & -2.364 & 45 & 6.735 & 37.53 \\
\hline o50_n16_q3_m4_121 & ils-ni & -2.692 & -2.368 & -2.028 & 165.385 & 6.774 & 30.25 \\
\hline o50_n16_q3_m4_121 & ils-ns & 2.421 & 2.421 & 2.421 & 0.000 & 12.384 & 7.29 \\
\hline o50_n16_q3_m4_121 & ils-nr & -1.881 & -1.480 & -1.153 & 173.145 & 7.664 & 11.050 \\
\hline $\mathrm{o} 50 \_\mathrm{n} 16 \_\mathrm{q} 3 \_\mathrm{m} 4 \_121$ & ils-nsi & -2.692 & -2.421 & & & 74 & 34.830 \\
\hline o50_n16_q3_m4_121 & ils-nsr & -2.785 & -2.527 & -2.188 & 114.464 & 6.671 & 34.658 \\
\hline o50_n16_q3_m4_121 & ils-os & -1.706 & -1.424 & -0.891 & 161.060 & 7.856 & 12.115 \\
\hline o50_n16_q3_m4_121 & ils-or & 1.809 & 1.809 & 1.809 & 0.000 & 11.713 & 7.147 \\
\hline o50_n16_q3_m4_122 & $i l_{\mathrm{c}}$ & -2.882 & -2.575 & -2.221 & 195.584 & 12.743 & 46.13 \\
\hline o50_n $16 \_\mathrm{q} 3 \_\mathrm{m} 4 \_122$ & ils_ni & -2.908 & -2.533 & & & & 34.56 \\
\hline o50_n16_q3_m4_122 & ils-ns & 0.549 & 0.549 & 0.549 & 0.000 & 16.726 & 8.04 \\
\hline o50_n16_q3_m4_122 & ils-nr & -2.268 & -1.848 & -1.481 & 291.995 & 13.456 & 12.535 \\
\hline o50_n16_q $3 \_m 4 \_122$ & ils-nsi & -2.842 & -2.601 & -2.275 & 188.916 & 12.789 & 44.082 \\
\hline o50_n16_q3_m4_122 & ils-nsr & -2.762 & -2.586 & -2.474 & 107.652 & 12.882 & 44.717 \\
\hline o50_n $16 \_\mathrm{q} 3 \_\mathrm{m} 4 \_122$ & ils-os & -2.607 & -1.836 & -1.458 & & 13.062 & 13.624 \\
\hline o50_n $16 \_\mathrm{q} 3 \_\mathrm{m} 4 \_122$ & ils-or & 0.591 & 0.591 & 0.591 & 0.000 & 16.775 & 8.219 \\
\hline o50_n16_q3_m4_131 & ils & -1.640 & -1.596 & -1.414 & 64.291 & 5.490 & 40.48 \\
\hline o50_n16_q3_m4_131 & ils-ni & -1.633 & -1.550 & -1.358 & 80.424 & 5.497 & 33.13 \\
\hline o50_n16_q3_m4_131 & ils-ns & 4.561 & 4.561 & 4.561 & 0.000 & 12.141 & 7.03 \\
\hline o50_n16_q3_m4_131 & ils-nr & -1.300 & -0.845 & -0.332 & 293.344 & 5.854 & 11.393 \\
\hline o50_n16_q $3 \_m 4 \_131$ & ils-nsi & -1.811 & -1.660 & -1.558 & 66.000 & 5.307 & 37.760 \\
\hline o50_n16_q $3 \_m 4 \_131$ & ils-nsr & -1.740 & -1.639 & -1.516 & 61.654 & 5.383 & 38.415 \\
\hline o50_n16_q3_m4_131 & ils-os & -0.905 & -0.567 & -0.166 & 214.979 & 6.278 & 12.872 \\
\hline o50_n16_q3_m4_131 & ils-or & 2.068 & 2.068 & 2.068 & 0.000 & 9.467 & 7.470 \\
\hline o50_n16_q3_m4_132 & ils & -1.158 & -0.889 & -0.650 & 156.502 & 6.718 & 48.66 \\
\hline o50_n16_q3_m4_132 & ils-ni & -1.176 & -0.839 & -0.661 & 163.383 & 6.698 & 40.52 \\
\hline o50_n16_q3_m4_132 & ils-ns & 1.487 & 1.487 & 1.487 & 0.000 & 9.573 & 7.60 \\
\hline o50_n16_q3_m4_132 & ils-nr & -0.390 & -0.164 & 0.133 & 168.434 & 7.547 & 13.114 \\
\hline
\end{tabular}




\begin{tabular}{|c|c|c|c|c|c|c|c|}
\hline Instance & Method & $R P D^{-}$ & $\overline{R P D}$ & $R P D^{+}$ & $\sigma_{R P D}$ & $R P D_{L B}^{-}$ & $\overline{\text { Time }}$ \\
\hline o50_n16_q3_m4_132 & ils-nsi & -1.085 & -0.913 & -0.663 & 125.675 & 6.796 & 45.335 \\
\hline o50_n16_q $3 \_m 4 \_132$ & ils-nsr & -1.046 & -0.828 & -0.602 & 129.029 & 6.839 & 45.565 \\
\hline o50_n16_q3_m4_132 & ils-os & -0.297 & 0.008 & 0.320 & 228.688 & 7.648 & 14.435 \\
\hline o50_n16_q3_m4_132 & ils-or & 2.553 & 2.553 & 2.553 & 0.000 & 10.725 & 8.468 \\
\hline o50_n16_q3_m4_211 & ils & -6.248 & -5.834 & -5.454 & 244.691 & 9.214 & 35.26 \\
\hline o50_n16_q3_m4 211 & ils-ni & -5.851 & -5.489 & -5.179 & 202.163 & 9.676 & 24.62 \\
\hline o50_n16_q $3 \_m 4 \_211$ & ils-ns & -2.243 & -2.243 & -2.243 & 0.000 & 13.878 & 6.57 \\
\hline o50_n16_q3_m4_211 & ils-nr & -5.370 & -4.454 & -3.727 & 423.200 & 10.236 & 9.127 \\
\hline o50_n16_q $3 \_m 4 \_211$ & ils-nsi & -6.437 & -5.793 & -5.460 & 283.937 & 8.993 & 33.678 \\
\hline o50_n16_q3_m4_211 & ils-nsr & -6.183 & -5.849 & -5.329 & 216.258 & 9.289 & 32.621 \\
\hline o50_n16_q3_m4_211 & ils-os & -4.891 & -4.405 & -3.975 & 303.759 & 10.794 & 9.873 \\
\hline o50_n16_q $3 \_m 4 \_211$ & ils-or & -2.207 & -2.207 & -2.207 & 0.000 & 13.921 & 7.276 \\
\hline o50_n16_q3_m4_212 & ils & -7.918 & -7.668 & -7.287 & 171.049 & 15.508 & 34.20 \\
\hline o50_n16_q $3 \_\mathrm{m} 4 \_212$ & ils-ni & -7.703 & -7.404 & -7.146 & 184.134 & 15.779 & 23.91 \\
\hline o50_n16_q3_m4_212 & ils-ns & -2.976 & -2.976 & -2.976 & 0.000 & 21.707 & 7.31 \\
\hline o50_n16_q3_m4_212 & ils-nr & -6.895 & -6.383 & -5.947 & 267.820 & 16.792 & 9.764 \\
\hline o50_n16_q3_m4_212 & ils-nsi & -7.934 & -7.497 & -7.183 & 216.401 & 15.488 & 32.247 \\
\hline o50_n16_q3_m4_212 & ils-nsr & -7.910 & -7.615 & -7.230 & 174.608 & 15.518 & 32.735 \\
\hline o50_n16_q $3 \_\mathrm{m} 4 \_212$ & ils-os & -7.004 & -6.234 & -5.679 & 363.633 & 16.655 & 10.777 \\
\hline o50_n16_q $3 \_\mathrm{m} 4 \_212$ & ils-or & -2.777 & -2.777 & -2.777 & 0.000 & 21.958 & 7.257 \\
\hline o50_n16_q3_m4_221 & ils & -2.899 & -2.812 & -2.571 & 112.682 & 9.361 & 41.81 \\
\hline o50_n16_q $3 \_m 4 \_221$ & ils-ni & -2.835 & -2.676 & -2.239 & 235.512 & 9.433 & 33.69 \\
\hline o50_n16_q3_m4_221 & ils-ns & 2.361 & 2.361 & 2.361 & 0.000 & 15.285 & 7.47 \\
\hline o50_n16_q $3 \_m 4 \_221$ & ils-nr & -2.159 & -1.779 & -1.501 & 226.160 & 10.194 & 11.085 \\
\hline o50_n16_q3_m4_221 & ils-nsi & -2.899 & -2.791 & -2.658 & 84.367 & 9.361 & 40.155 \\
\hline o50_n16_q3_m4_221 & ils-nsr & -2.899 & -2.815 & -2.461 & 144.565 & 9.361 & 40.003 \\
\hline o50_n16_q3_m4_221 & ils-os & -1.859 & -1.569 & -1.213 & 233.009 & 10.531 & 11.862 \\
\hline o50_n16_q3_m4_221 & ils-or & 0.304 & 0.304 & 0.304 & 0.000 & 12.968 & 7.519 \\
\hline o50_n16_q $3 \_m 4 \_222$ & ils & -3.672 & -3.344 & -2.878 & 251.539 & 19.448 & 32.01 \\
\hline o50_n16_q3_m4_222 & ils-ni & -4.176 & -3.435 & -3.145 & 321.616 & 18.824 & 26.33 \\
\hline o50_n16_q $3 \_m 4 \_222$ & ils-ns & 2.181 & 2.181 & 2.181 & 0.000 & 26.706 & 7.91 \\
\hline o50_n16_q3_m4_222 & ils-nr & -3.056 & -2.169 & -1.243 & 708.794 & 20.212 & 8.719 \\
\hline o50_n16_q3_m4_222 & ils-nsi & -3.703 & -3.262 & -2.890 & 263.043 & 19.410 & 31.229 \\
\hline o50_n16_q $3 \_m 4 \_222$ & ils-nsr & -3.872 & -3.389 & -2.946 & 200.911 & 19.200 & 30.214 \\
\hline o50_n16_q3_m4_222 & ils-os & -2.491 & -1.849 & -1.282 & 457.119 & 20.913 & 9.230 \\
\hline o50_n $16 \_\mathrm{q} 3 \_\mathrm{m} 4 \_222$ & ils-or & 2.121 & 2.121 & 2.121 & 0.000 & 26.632 & 7.544 \\
\hline o50_n16_q3_m4_231 & ils & -5.078 & -4.912 & -4.616 & 142.526 & 3.553 & 43.74 \\
\hline o50_n16_q3_m4_231 & ils-ni & -5.211 & -4.957 & -4.706 & 162.716 & 3.408 & 37.34 \\
\hline o50_n16_q $3 \_m 4 \_231$ & ils-ns & -0.127 & -0.127 & -0.127 & 0.000 & 8.954 & 7.34 \\
\hline o50_n16_q3_m4_231 & ils-nr & -4.705 & -4.178 & -3.854 & 202.720 & 3.960 & 13.033 \\
\hline o50_n16_q $3 \_m 4 \_231$ & ils-nsi & -5.058 & -4.944 & -4.740 & 96.526 & 3.575 & 40.507 \\
\hline
\end{tabular}




\begin{tabular}{|c|c|c|c|c|c|c|c|}
\hline Instance & Method & $R P D^{-}$ & $\overline{R P D}$ & $R P D^{+}$ & $\sigma_{R P D}$ & $R P D_{L B}^{-}$ & $\overline{\text { Time }}$ \\
\hline o50_n16_q3 3_m4_231 & ils-nsr & -5.119 & -4.974 & -4.764 & 95.978 & 3.508 & 41.505 \\
\hline o50_n16_q3 3_m4_231 & ils-os & -4.281 & -3.960 & -3.650 & 185.122 & 4.422 & 14.263 \\
\hline o50_n16_q3_m4_231 & ils-or & -0.313 & -0.313 & -0.313 & 0.000 & 8.751 & 8.013 \\
\hline o50_n16_q3_m4_232 & ils & -1.468 & -1.233 & -0.862 & 263.335 & 13.337 & 31.36 \\
\hline o50_n16_q3_m4_232 & ils-ni & -1.542 & -1.243 & -1.030 & 242.128 & .253 & 21.43 \\
\hline o50_n16_q3_m4_232 & ils-ns & -0.092 & -0.092 & -0.092 & 0.000 & 14.921 & 7.24 \\
\hline o50_n16_q3_m4_232 & ils-nr & -0.975 & 0.047 & 0.717 & 558.439 & 13.904 & 8.730 \\
\hline o50_n16_q3_m4_232 & ils-nsi & -1.558 & -1.345 & -0.971 & 236.597 & 13.234 & 29.915 \\
\hline o50_n16_q3_m4_232 & ils-nsr & -1.499 & -1.288 & -1.001 & 218.837 & 13.302 & 29.412 \\
\hline o50_n16_q3_m4_232 & ils-os & -0.920 & 0.189 & 0.711 & 578.867 & 13.967 & 9.242 \\
\hline o50_n16_q3_m4_232 & ils-or & 2.989 & 2.989 & 2.989 & 0.000 & 18.464 & 8.272 \\
\hline o50_n16_q3_m8_111 & ils & -3.743 & -3.454 & -3.092 & 76.359 & 9.192 & 31.53 \\
\hline o50_n16_q3_m8_111 & ils-ni & -3.848 & -3.214 & -2.498 & 19.531 & 9.074 & 26.32 \\
\hline o50_n16_q3_m8_111 & ils-ns & 1.166 & 1.166 & 1.166 & 0.000 & 4.762 & 6.80 \\
\hline o50_n16_q3_m8_111 & ils-nr & -2.179 & -1.513 & -0.855 & 2.625 & 10.967 & 8.740 \\
\hline o50_n16_q3_m8_111 & ils-nsi & -3.615 & -3.398 & -3.211 & 52.243 & 9.337 & 30.892 \\
\hline o50_n16_q3_m8_111 & ils-nsr & -3.796 & -3.353 & -2.807 & 116.083 & 9.133 & 31.069 \\
\hline o50_n16_q3_m8_111 & ils-os & -2.277 & -1.673 & -1.035 & 145.861 & 10.855 & 9.085 \\
\hline o50_n16_q3_m8_111 & ils-or & 2.048 & 2.048 & 2.048 & 0.000 & 762 & 7.087 \\
\hline o50_n16_q3_m8_112 & ils & 1.021 & -10.658 & 0.097 & 126.120 & 28 & 40.14 \\
\hline o50_n16_q3_m8_112 & ils-ni & -11.112 & -10.617 & -10.216 & 153.189 & 20.705 & 31.70 \\
\hline o50_n16_q3_m8_112 & ils-ns & -5.829 & -5.829 & -5.829 & 0.000 & 27.879 & 8.32 \\
\hline o50_n16_q3_m8_112 & ils-nr & -10.287 & -9.628 & -8.974 & .999 & 21.826 & 10.348 \\
\hline o50_n16_q3_m8_112 & & & & & & & 55 \\
\hline o50_n16_q3_m8_112 & ils-nsr & -11.429 & -10.820 & -10.362 & 162.580 & 20.275 & 38.522 \\
\hline o50_n16_q3_m8_112 & ils-os & -9.857 & -9.322 & -8.796 & 179.366 & 22.409 & 10.772 \\
\hline o50_n16_q3_m8_112 & ils-or & -5.101 & -5.101 & -5.101 & 0.000 & 28.868 & 9.051 \\
\hline o50_n16_q3 $3 \_m 8 \_121$ & $i l_{c}$ & -5.219 & -5.098 & -4.912 & 28.837 & 8.051 & 38.18 \\
\hline o50_n16_q3_m8_121 & il & -5.200 & -4.971 & -4.545 & 66.853 & 73 & 28.35 \\
\hline o50_n16_q3_m8_121 & ils-ns & -1.850 & -1.850 & -1.850 & 0.000 & 11.891 & 7.31 \\
\hline o50_n16_q3_m8_121 & ils-nr & -4.392 & -4.036 & -3.447 & 98.546 & 8.993 & 10.516 \\
\hline o50_n16_q3_m8_121 & ils-nsi & -5.291 & -5.097 & -4.921 & 43.001 & 7.969 & 36.568 \\
\hline o50_n16_q3_m8_121 & ils-nsr & -5.212 & -5.033 & -4.758 & 48.356 & 8.058 & 35.565 \\
\hline o50_n16_q3_m8_121 & ils-os & -4.145 & -3.618 & -2.893 & 125.012 & 9.275 & 11.096 \\
\hline o50_n16_q3_m8_121 & ils-or & -1.850 & -1.850 & -1.850 & 0.000 & 11.891 & 7.547 \\
\hline o50_n16_q3_m8_122 & ils & -5.766 & -5.026 & -4.597 & 163.812 & 15.060 & 41.48 \\
\hline o50_n16_q3_m8_122 & ils-ni & -5.151 & -4.615 & -4.336 & 127.789 & 15.811 & 30.73 \\
\hline o50_n16_q3_m8_122 & ils-ns & -0.957 & -0.957 & -0.957 & 0.000 & 20.932 & 8.08 \\
\hline o50_n16_q3_m8_122 & ils-nr & -4.350 & -3.674 & -3.317 & 171.749 & 16.788 & 9.962 \\
\hline o50_n16_q3_m8_122 & ils-nsi & -5.307 & -4.956 & -4.485 & 120.964 & 15.620 & 39.283 \\
\hline o50_n16_q3_m8_122 & ils-nsr & -5.407 & -4.975 & -4.611 & 114.504 & 15.498 & 38.406 \\
\hline
\end{tabular}




\begin{tabular}{|c|c|c|c|c|c|c|c|}
\hline Instance & Method & $R P D^{-}$ & $\overline{R P D}$ & $R P D^{+}$ & $\sigma_{R P D}$ & $R P D_{L B}^{-}$ & $\overline{\text { Time }}$ \\
\hline o50_n16_q3_m8_122 & ils-os & -3.699 & -3.306 & -3.070 & 94.883 & 17.584 & 10.677 \\
\hline o50_n16_q3_m8_122 & ils-or & -1.473 & -1.473 & -1.473 & 0.000 & 20.301 & 8.311 \\
\hline o50_n16_q3_m8_131 & ils & -3.257 & -3.008 & -2.572 & 151.353 & 3.866 & 34.79 \\
\hline o50_n16_q3_m8_131 & ils-ni & -3.207 & -2.738 & -2.239 & 171.065 & 3.919 & 29.12 \\
\hline o50_n16_q3_m8_131 & ils-ns & 1.870 & 1.870 & 1.870 & 0.000 & 9.370 & 7.71 \\
\hline o50_n16_q $3 \_m 8 \_131$ & ils-nr & -2.631 & -1.983 & -1.235 & 297.577 & 4.538 & 8.861 \\
\hline o50_n16_q3_m8_131 & ils-nsi & -3.459 & -2.930 & -2.214 & 209.763 & 3.648 & 33.622 \\
\hline o50_n $16 \_\mathrm{q} 3 \_\mathrm{m} 8 \_131$ & ils-nsr & -3.156 & -2.823 & -2.377 & 175.026 & 3.974 & 32.419 \\
\hline o50_n16_q3_m8_131 & ils-os & -1.995 & -1.647 & -1.197 & 172.068 & 5.220 & 9.117 \\
\hline o50_n16_q3_m8_131 & ils-or & 1.870 & 1.870 & 1.870 & 0.000 & .370 & 7.839 \\
\hline o50_n16_q3_m8_132 & ils & -5.318 & -4.666 & -4.230 & 218.757 & 15.355 & 39.37 \\
\hline o50_n $16 \_\mathrm{q} 3 \_\mathrm{m} 8 \_132$ & ils-ni & -4.829 & -4.452 & -4.054 & 163.768 & 15.950 & 29.51 \\
\hline o50_n16_q3_m8_132 & ils-ns & -1.407 & -1.407 & -1.407 & 0.000 & 20.119 & 7.66 \\
\hline o50_n16_q3_m8_132 & ils-nr & -4.376 & -3.336 & -2.831 & & 502 & 11.271 \\
\hline o50_n16_q3_m8_132 & ils-nsi & -5.046 & -4.599 & -4.204 & 164 & 686 & 36.750 \\
\hline o50_n16_q3_m8_132 & ils-nsr & -4.822 & -4.501 & -4.070 & 151.585 & 15.959 & 37.188 \\
\hline o50_n16_q3_m8_132 & ils-os & -3.467 & -3.130 & -2.737 & 162.713 & 17.610 & 12.039 \\
\hline o50_n16_q $3 \_m 8 \_132$ & ils-or & -0.396 & -0.396 & -0.396 & 0.000 & 21.351 & 8.466 \\
\hline o50_n16_q3_m8_211 & ils & & -4.566 & & & 64 & 43.67 \\
\hline o50_n16_q3_m8_211 & ils-ni & -4.783 & -4.393 & & 111.499 & 169 & 33.16 \\
\hline o50_n $16 \_\mathrm{q} 3 \_\mathrm{m} 8 \_211$ & ils-ns & -0.939 & -0.939 & -0.939 & 0.000 & 16.698 & 6.89 \\
\hline o50_n $16 \_\mathrm{q} 3 \_\mathrm{m} 8 \_211$ & ils-nr & -4.854 & -3.779 & -3.305 & 223.031 & 12.086 & 13.432 \\
\hline o50_n $16 \_\mathrm{q} 3 \_\mathrm{m} 8 \_211$ & ils-nsi & -4.668 & -4.432 & -4.219 & 72.437 & 12.305 & 41.121 \\
\hline o50_n16_q3_m8_211 & ils-nsr & -4.808 & -4.412 & -3.938 & & 40 & 41.679 \\
\hline o50_n16_q3_m8_211 & ils-os & -3.710 & -3.142 & -2.573 & & 13.433 & 14.374 \\
\hline o50_n16_q3_m8_211 & ils-or & -0.939 & -0.939 & -0.939 & 0.000 & 16.698 & 7.528 \\
\hline o50_n $16 \_\mathrm{q} 3 \_\mathrm{m} 8 \_212$ & ils & -9.940 & -9.601 & -9.287 & 129.294 & 19.713 & 49.55 \\
\hline o50_n $16 \_\mathrm{q} 3 \_\mathrm{m} 8 \_212$ & ils-ni & -9.781 & -9.505 & -9.295 & 114.855 & 19.924 & 40.55 \\
\hline o50_n16_q3_m8_212 & ils-ns & -3.914 & -3.914 & -3.914 & 0.000 & 27.722 & 8.25 \\
\hline o50_n16_q3_m8_212 & ils-nr & -9.119 & -8.617 & -7.855 & 254.471 & 20.804 & 12.662 \\
\hline o50_n16_q3_m8_212 & ils-nsi & -10.293 & -9.718 & -9.227 & 195.837 & 19.243 & 46.818 \\
\hline o50_n16_q3_m8_212 & ils-nsr & -9.967 & -9.680 & -9.421 & 94.826 & 19.677 & 46.563 \\
\hline o50_n $16 \_\mathrm{q} 3 \_\mathrm{m} 8 \_212$ & ils-os & -9.527 & -8.689 & -7.938 & 336.753 & 20.261 & 13.632 \\
\hline o50_n16_q3_m8_212 & ils-or & -3.914 & -3.914 & -3.914 & 0.000 & 27.722 & 7.972 \\
\hline o50_n16_q3_m8_221 & ils & -5.368 & -4.980 & -4.742 & 82.884 & 8.221 & 41.64 \\
\hline o50_n16_q3_m8_221 & ils-ni & -5.368 & -4.878 & -4.669 & 71.949 & 8.221 & 29.50 \\
\hline o50_n16_q3_m8_221 & ils-ns & -1.131 & -1.131 & -1.131 & 0.000 & 13.067 & 7.81 \\
\hline o50_n16_q3_m8_221 & ils-nr & -4.514 & -4.033 & -3.744 & 105.938 & 9.198 & 10.625 \\
\hline o50_n16_q3_m8_221 & ils-nsi & -5.303 & -5.088 & -4.852 & 53.957 & 8.295 & 39.345 \\
\hline o50_n16_q3_m8_221 & ils-nsr & -5.495 & -5.146 & -4.646 & 83.507 & 8.076 & 38.835 \\
\hline o50_n16_q3_m8_221 & ils-os & -4.170 & -3.807 & -3.425 & 79.192 & 9.592 & 11.177 \\
\hline
\end{tabular}




\begin{tabular}{lccccrrr}
\hline Instance & Method & $R P D^{-}$ & $\overline{R P D}$ & $R P D^{+}$ & \multicolumn{1}{c}{$\sigma_{R P D}$} & $R P D_{L B}^{-}$ & $\overline{T i m e}$ \\
\hline o50_n16_q3_m8_221 & ils-or & -1.181 & -1.181 & -1.181 & 0.000 & 13.009 & 7.587 \\
o50_n16_q3_m8_222 & ils & -7.461 & -7.219 & -6.823 & 119.918 & 15.205 & 42.56 \\
o50_n16_q3_m8_222 & ils-ni & -7.389 & -6.958 & -6.661 & 127.207 & 15.296 & 29.09 \\
o50_n16_q3_m8_222 & ils-ns & -1.959 & -1.959 & -1.959 & 0.000 & 22.055 & 7.22 \\
o50_n16_q3_m8_222 & ils-nr & -6.307 & -5.661 & -5.104 & 187.633 & 16.642 & 10.713 \\
o50_n16_q3_m8_222 & ils-nsi & -7.578 & -7.154 & -6.147 & 227.699 & 15.060 & 40.133 \\
o50_n16_q3_m8_222 & ils-nsr & -7.597 & -7.201 & -6.989 & 124.603 & 15.036 & 40.407 \\
o50_n16_q3_m8_222 & ils-os & -6.276 & -5.459 & -5.018 & 197.692 & 16.681 & 11.245 \\
o50_n16_q3_m8_222 & ils-or & -3.366 & -3.366 & -3.366 & 0.000 & 20.304 & 7.834 \\
o50_n16_q3_m8_231 & ils & -2.798 & -2.252 & -1.818 & 154.825 & 3.081 & 48.65 \\
o50_n16_q3_m8_231 & ils-ni & -2.461 & -2.230 & -1.930 & 83.476 & 3.438 & 41.48 \\
o50_n16_q3_m8_231 & ils-ns & 2.348 & 2.348 & 2.348 & 0.000 & 8.538 & 7.61 \\
o50_n16_q3_m8_231 & ils-nr & -1.805 & -1.558 & -1.291 & 94.792 & 4.134 & 13.125 \\
o50_n16_q3_m8_231 & ils-nsi & -2.230 & -2.138 & -2.036 & 31.214 & 3.683 & 46.475 \\
o50_n16_q3_m8_231 & ils-nsr & -2.574 & -2.278 & -2.126 & 83.361 & 3.318 & 47.264 \\
o50_n16_q3_m8_231 & ils-os & -1.951 & -1.533 & -1.036 & 153.285 & 3.979 & 13.975 \\
o50_n16_q3_m8_231 & ils-or & 1.045 & 1.045 & 1.045 & 0.000 & 7.155 & 7.453 \\
o50_n16_q3_m8_232 & ils & -2.020 & -1.799 & -1.586 & 95.867 & 7.572 & 29.66 \\
o50_n16_q3_m8_232 & ils-ni & -2.232 & -1.892 & -1.424 & 125.565 & 7.339 & 24.59 \\
o50_n16_q3_m8_232 & ils-ns & 1.752 & 1.752 & 1.752 & 0.000 & 11.713 & 7.71 \\
o50_n16_q3_m8_232 & ils-nr & 0.694 & 1.443 & 1.947 & 268.860 & 10.551 & 7.405 \\
o50_n16_q3_m8_232 & ils-nsi & -2.102 & -1.837 & -1.365 & 149.459 & 7.483 & 27.476 \\
o50_n16_q3_m8_232 & ils-nsr & -2.059 & -1.866 & -1.554 & 98.334 & 7.530 & 26.743 \\
o50_n16_q3_m8_232 & ils-os & 1.058 & 1.458 & 2.044 & 226.008 & 10.951 & 7.655 \\
o50_n16_q3_m8_232 & ils-or & 2.024 & 2.024 & 2.024 & 0.000 & 12.012 & 8.406
\end{tabular}

